\title{
Temporal Responses of Chemically Diverse Sensor Arrays for Machine OLFACTION USING ARTIFICIAL INTELLIGENCE
}

By

Shaun Kenneth Ryman

A Thesis submitted to the Faculty of Graduate Studies of

The University of Manitoba

In partial fulfillment of the requirements of the degree of

\section{MASTER OF SCIENCE}

Department of Chemistry

University of Manitoba

Winnipeg

Copyright (C) by Shaun K. Ryman 


\begin{abstract}
The human olfactory system can classify new odors in a dynamic environment with varying odor complexity and concentration, while simultaneously reducing the influence of stable background odors. Replication of this capability has remained an active area of research over the past 3 decades and has great potential to advance medical diagnostics, environmental monitoring and industrial monitoring, among others. New methods for rapid dynamic temporal evaluation of chemical sensor arrays for the monitoring of analytes is explored in this work. One such method is high and low bandpass filtering of changing sensor responses; this is applied to reduce the effects of background noise and sensor drift over time. Processed sensor array responses, coupled with principal component analysis (PCA), will be used to develop a novel approach to classify odors in the presence of changing sensor responses associated with evolving odor concentrations. These methods will enable the removal of noise and drift, as well as facilitating the normalization to decouple classification patterns from intensity; lastly, PCA and artificial neural networks (ANNs) will be used to demonstrate the capability of this approach to function under dynamic conditions, where concentration is changing temporally.
\end{abstract}




\section{Acknowledgements}

There are many people whom I would like to acknowledge that have made important impacts on my experiences as a graduate student. I have had a lot of support and mentorship as a graduate student, from a great deal of faculty at the University of Manitoba as well from my fellow graduate students. I would like to thank past and present members of the Freund group: Dr. Mike McDonald, Dr. Ramesh Kumar, Dr. Kevin McEleney, Akin Iyogun, Jared Bruce, Megan, McClarty, Patrich Geisbrecht, Ehsan Tahmasebian, Dr. Onkar Kang, Heather Cavers. I would also like to thank Joey Lussier. Every one of them has made being graduate student an enjoyable experience in spite of the tough times.

I have a lot of gratitude and high regard for such an amazing supervisor, Dr. Michael Freund who initially accepted me into his group over the summer as an undergraduate research student. I stayed on with his guidance foe a research course, and lastly he agreed to suffer through a M.Sc. with me. His insights and extensive knowledge guided and built confidence in my career as a scientist.

I would like to thank my Committee members Dr. Hélène Perreault and Dr. Neil D. B. Bruce for their assistance and attention in crafting this work. Dr. Neil D. B. Bruce had graciously set aside a lot of his time to assist me in building and tying together some of the more complex details in this work.

I would like to thank my Mom (Sylvia), my Dad (Ken), Sister (Shannon), Sister (Shayla), Sister (Shelby) and Sister (Sheena). They have always been there to encourage and support me in my endeavors. I cannot thank them enough. 
Last but not least I would like to thank my partner Kaitlyn for her seemingly endless care and support in helping me push through. 


\section{Table of Contents}

$\begin{array}{lr}\text { ABSTRACT } & 2\end{array}$

ACKNOWLEDGEMENTS 3

\begin{tabular}{ll} 
THESIS STATEMENT & 7 \\
\hline
\end{tabular}

\begin{tabular}{lr} 
LIST OF TABLES & 8 \\
\hline
\end{tabular}

LIST OF FIGURES 9

LIST OF COPYRIGHT MATERIALS 14

CHAPTER 1: INTRODUCTION TO MACHINE OLFACTION

1.1 - IMPORTANCE OF CHEMICAL SENSING

1.1.1 - MAMMALIAN OLFACTION

1.2 - SENSOR ARRAYS $\quad 21$

1.2.1 - POLYMERS 24

1.2.2 - SENSING MECHANISMS

1.3 - Data ANALYSIS METHODS

1.3.1 - PRINCIPAL COMPONENT ANALYSIS (PCA) 27

1.3.2 - ARTIFICIAL NEURAL NETWORKS (ANNS) 28

CHAPTER 2: EXPERIMENTAL TECHNIQUES 33

2.1 - Making Carbon Black - ORganic Polymer Composite Sensors 33

2.2 - SOLVENTS

2.3 - INITIAL CHARACTERIZATION OF THE SENSOR ARRAY

2.4 - TEMPORAL ANALYSIS $\quad 34$

2.5 - DYNAMIC PATTERN RECOGNITION

2.6 - ANN ANALYSIS: UNSUPERVISED METHOD

2.7 - ANN: SUPERVISED METHOD $\quad 36$

CHAPTER 3: CHARACTERIZATION \& QUANTIFICATION 38

3.1 - NORMALIZED RESISTANCE $(\Delta R / R) \quad 38$

3.2 - $\triangle$ R/R PCA CHARACTERIZATION $\quad 42$

3.3 - CONCENTRATION RAMPING AND INTENSITY FACTOR

3.4 - QUANTITATIVE PCA CHARACTERIZATION 48

3.5 - SENSITIVITY

3.6 - CONCLUSIONS \& FUTURE WORK

CHAPTER 4: TEMPORAL ANALYSIS $\quad 57$

4.1 - TIME CONSTANTS

4.2 - TEMPORAL RESPONSE

4.3 - INTERACTIONS $\quad 64$

4.4 - DYNAMIC EQUILIBRATED RESPONSES

4.4.1 - CONSTANT TOTAL FLOW RATE $(400 \rightarrow 420$ SCCM) 66

4.1.2 - CONSTANT TOTAL Flow RATE (400 SCCM) 69 
4.4.3 - TIME DELAY IN RESPONSE

4.5 - MULTIPLE ODORS

4.6 - FILTERING

4.7 - CONCLUSIONS AND FUTURE WORK

CHAPTER 5: ANN \& REAL-TIME ANALYSIS $\quad 83$

5.1 - ANN \& SPARSE FILTERING

5.2 - REAL-TIME PCA \& ANN

5.3 - REAL-TIME ANN ANALYSIS $\quad 92$

5.4 - AdDITIONAL CONSIDERATIONS $\quad 92$

5.4 - CONCLUSIONS

CHAPTER 7: CONCLUSIONS AND FUTURE WORK

\begin{tabular}{lr} 
WORKS CITED & 99 \\
\hline
\end{tabular} 


\section{Thesis Statement}

As of yet, there is no practical approach to monitoring, in real time and under dynamic conditions, the surrounding chemical environment using vapor sensor arrays. By using novel analysis methods of filtering, pattern recognition and pattern normalization, there is a greater capability for monitoring and understanding the temporal response; with this, vapors may be identified and quantified in real time, using a carbon black - organic polymer sensor array. 


\section{List of Tables}

Table1.2.1: List of polymers used in carbon black-composite sensor array

Table 4.1.1: Average rising time constants for all carbon black-organic polymer sensors in minutes. Each sensor type consisted of 7 replicate sensors with 5 exposures each.

Table 4.1.2: Calculated Pearson's chi-squared test values. $X^{2}$ values less than 0.3 are considered to provide a good fit of the theoretical simulation to the experimental data

Table 4.2.1: Time before clustering within principal component space for PCA and normalized pca $(P C A+I F)$.

Table 4.3.0: Solvation parameters of different solvents used in all the experiments. ${ }^{1} \boldsymbol{R}^{2}$ is the excess molar refraction that can be calculated form refractive index or can rather be easily estimated and ranges from -0.240 (Isoflurane) $\rightarrow 2.29$ (Anthracene). $\mathbf{p} 2 \boldsymbol{H}$ is the solute dipolarity-polarizability obtained to date from gas-liquid chromatography of solutes on polar stationary phases and ranges from -0.20 (Sulfur hexafluoride) $\rightarrow 1.72$ (4Nitrophenol). $\mathbf{a} 2 \boldsymbol{H}$ is the solute hydrogen bond acidity and ranges from 0.00 (Cyclopentane) $\rightarrow 0.95$ (Trichloroacetic acid). $\mathbf{b} 2 \mathbf{H}$ is the solute hydrogen bond basicity and ranges from 0.00 (Cyclopentane) $\rightarrow 1.21$ (Tri-n-butyl phosphate). lastly logL is the solute-gas-hexadecane partition coefficient at $298 \mathrm{~K}$ and ranges from-0.120(Sulfur

hexafluoride) $\rightarrow 7.632$ (Phenanthrene) .......................................................................................66 


\section{List of Figures}

Figure 1.1.1: Human olfaction in the detection of vapors: 1) Olfactory epithelium 2) Olfactory bulb 3) Olfactory cortex 4) Thalamus 5) Orbitofrontal cortex. (Copyright 2010 J. Exp. Brain. Res.)

Figure 1.2.1: Gold plated interdigitated array. Sensor size is $8 \mathrm{~mm}$ tall by $3 \mathrm{~mm}$ wide

Figure 1.2.2: Reversible vapor absorption in carbon black-organic polymer composite sensors.

Figure 1.3.2: General ANN architecture .28

Figure 1.3.4: Left: Sparse filtering showing two features $\left(f_{1}, f_{2}\right)$ and two examples (red and green). Each example is first projected onto the 12-ball and then optimized for sparseness. The 12-ball is shown together with level sets of the l1-norm. Notice that the sparseness of the features (in the 11 sense) is maximized when the examples are on the axes. Right: Competition between features due to normalization. We show one example where only $f 1$ is increased. Notice that even though only $f$ is increased, the normalized value of the second feature, $\hat{f}$ decreases. (Copyright 2011 Jiquan Ngiam et al.).........32

Figure 3.1.0: Temporal resistance response of Carbon black-Poly(ethylene oxide) sensor to toluene(blue), dichloromethane (red), methanol (green), acetonitrile (purple) and water (orange) over 20min on/off intervals. Data recorded at $25.0 \pm 0.2$ ${ }^{\circ} \mathrm{C}, 400 \mathrm{sccm}$ nitrogen and $5 \mathrm{vol} \%$ analyte pressure.

Figure 3.1.1: How $\Delta R / R$ is measured using standard methods. The baseline resistance is represented as $R$, the equilibrated peak response is the maximum at the end of exposure then subtracting the baseline resistance $(\Delta R)$.

Figure 3.1.2: $\Delta R / R$ equilibrated response to vapors of toluene (blue), dichloromethane (red), methanol (green) and acetonitrile (purple). The average $\Delta R / R$ was taken over seven identical sensors with 5 exposures each. Exposures were random 20 min on/off exposures.

Figure 3.2.0: PCA response using the sensor arrays equilibrated $\Delta R / R$ response of toluene (blue), dichloromethane (red), methanol (green), acetonitrile (purple) and water (cyan). The vapor pressure of each analyte is $5 \% \mathrm{P} / \mathrm{P}^{0}$ for 20 minute on/off exposures with 60 minutes separations between different vapors.

Figure 3.2.1: $P C A$ response using the sensor arrays equilibrated $\Delta R / R$ response of toluene (blue), dichloromethane (red), methanol (green), acetonitrile (purple). The vapor pressure of each analyte is $5 \% \mathrm{P} / \mathrm{P}^{0}$ for 20 minute on/off exposures with 60 minutes separations between different vapors. 
Figure 3.3.0: Temporal resistance response of Carbon black-Poly(vinyl butyral) sensor to toluene(blue), dichloromethane (red), methanol (green), acetonitrile (purple) over 20min on/off intervals. Data recorded at $25.0 \pm 0.2^{\circ} \mathrm{C}, 400 \mathrm{sccm}$ nitrogen and 0.5, 1.0, $1.5,2.0,2.5,3.0,5.0,10.0$ and $20.0 \% \mathrm{P} / \mathrm{P}^{0}$ analyte pressure.

Figure 3.3.1: $\Delta R / R$ values for methanol with increasing concentration. Darker bars indicate increasing concentration corresponding to $0.5,1.0,1.5,2.0,2.5,3.0,5.0,10.0$ and $20.0 \% \mathrm{P} / \mathrm{P}^{0}$ respectively.

Figure 3.3.2: Concentration independent component is shown above after separation of the intensity factor from the original $\Delta R / R$ data. Preprocessed data from Figure 3.3.1.

Figure 3.4.0: Principal component response of toluene (blue), dichloromethane (red), methanol (green) and acetonitrile (purple) with increasing concentration. Arrows show the direction of points with concentration increase of 0.5, 1.0, 1.5, 2.0, 2.5, 3.0, 5.0, 10.0 and $20.0 \% \mathrm{P} / \mathrm{P}^{0}$ respectively.

Figure 3.4.1: PC1 determined from normalized $\Delta R / R$ for methanol (Green), the green line represents the average response of $P C 1$ determined for normalized data, Intensity Factor (red) with an $R^{2}$ of 0.990. After the normalization of the response patterns, the increasing intensity factor as a function of increasing concentration, PC1 then becomes concentration independent.

Figure 3.4.2: Principal component response of the concentration independent pattern for toluene (blue), dichloromethane (red), methanol (green), acetonitrile (blue). Preprocessed data is shown in Figure 3.4.0. Four points are outside the range of this graph and excluded because they contain only random noise fluctuations. These points are dichloromethane at $2.0 \% \mathrm{P} / \mathrm{P}^{0}(0.84,1.69,3.07)$, acetonitrile $1.0 \% \mathrm{P} / \mathrm{P}^{0}(-0.49$, $0.51,-1.11)$, acetonitrile $1.5 \% P / P^{0}(-0.44,-0.85,-1.78)$, acetonitrile $3.0 \% P / P^{0}(-0.35$, $0.28,-0.64)$

Figure:3.4.3: Principal component response of the concentration independent pattern for toluene (blue), dichloromethane (red), methanol (green), acetonitrile (blue). Randomly scattered points below $2.5 \% \mathrm{P} / \mathrm{P}^{0}$ for toluene, dichloromethane, methanol and points below $5 \% \mathrm{P} / \mathrm{P}^{0}$ for acetonitrile have been omitted from the image. This is refined data from Figure 3.4.1.

Figure 3.5.1: Intensity factor of the sensor array as a function of concentration toluene(blue), dichloromethane(red), methanol(green) and acetonitrile(purple). Each vapor has a correlation coefficient $>0.947$.

Figure 4.1.1: Distribution of time constants within this sensor array for each vapor. 
Figure 4.2.1: Comparison of rates between PEO (Blue), PMVEMA(Orange). Black lines represent the theoretical fit between the data points. Error bars represent the standard deviation of 5 exposures to methanol.

Figure 4.2.2: $3 D$ plot of the averaged temporal response from the sensor array to the 4 vapors toluene(blue), dichloromethane(red), methanol(green) and acetonitrile(purple). Each point indicates 2 minutes up to the maximum $\Delta R / R$.

Figure 4.2.3: Normalized temporal response from Figure 4.2.2 using the Intensity Factor. Data points close to zero have been omitted from the image because they are randomly scattered within principal component space. The removed data points coincide with the first 4 minutes of analysis and the last 16 minutes after the equilibrated response......

Figure 4.2.4: Normalized temporal response from Figure 4.2.2 using the Intensity Factor. Data points close to zero have been omitted from the image because they are randomly scattered within principal component space.

Figure 4.4.1: a)[top left] Partial pressure applied over time to the sensor array. The concentration was gradually raised to a maximum of $5 \% \mathrm{P} / \mathrm{P}^{0}$ at a rate of $0.25 \% \mathrm{P} / \mathrm{P}^{0}$ every 5 min. b) [top right] Intensity factor changes with concentration. Rise (green) $R^{2}$ $=0.921$, fall (red) $R^{2}=0.847$. c) [bottom left] PC1 and PC2 response of $\Delta R / R$. d) [bottom right] Response of PC1 and PC2 for normalized data using the Intensity factor. Noisy, randomly scattered data points below the threshold intensity are marked in pink. Significant variance ensues below $2.5 \% \mathrm{P} / \mathrm{P}^{0}$ (3.95 ppt) at $25 \mathrm{C}^{\circ}$ and $1 \mathrm{~atm}$. Points at $(-0.049,1.20)$ and $(6.56,2.73)$ are randomly scattered within principal component space and have been omitted from the image for a better visual of the cluster.

Figure 4.4.2: a) [top] Intensity factor changes with concentration. Rise (blue) $R^{2}=$ 0.0.738, fall (red) $R^{2}=0.759$. b) [bottom left] $P C 1$ and $P C 2$ response of $\Delta R / R$. c) [bottom right] Response of PC1 and PC2 for normalized data using the Intensity factor. Points at $1.5 \% P / P^{0}(-13.173,30.878)$ on the rise as well as $0.25 \% P / P^{0}(-0.15539$, $5.2457)$ and $0.0 \% \mathrm{P} / \mathrm{P}^{0}(0.057149,-1.2535)$ on the return have been omitted from the figure as they are randomly scattered (pink points). (48 sensor array).

Figure 4.4.3: Theoretical modeling using the time constant with the slow changing flow rate used in the gradually changing concentration of methanol. The concentration profile (blue) is plotted over time. Time constants 1 minute (red), 6 minutes (green) and 12 minutes (purple) are used to calculate the $\Delta R / \Delta R_{\text {saturated }}$ over time.

Figure 4.5.1: Concentration profile of gradual exposures of toluene up to a concentration of $5 \% \mathrm{P} / \mathrm{P}^{0}$ with a fast changing exposure of methanol at $5 \% \mathrm{P} / \mathrm{P}^{0}$. 
Figure 4.5.2: Temporal response of intensity factor when exposed to a slow background of toluene up to $5 \% \mathrm{P} / \mathrm{P}^{0}$ and a quick changing vapor.....

Figure 4.5.3: Principal components of sensor array exposed to a slow background of toluene up to $5 \% \mathrm{P} / \mathrm{P}^{0}$ and a quick changing vapor.

Figure 4.5.4: a) [top] Concentration independent component of gradual exposures of toluene up to a concentration of $5 \% \mathrm{P} / \mathrm{P}^{0}$ with a fast changing exposure of methanol at $5 \% \mathrm{P} / \mathrm{P}^{\circ}$. b) [bottom] zoomed in profile of the normalized concentration independent pattern of $\boldsymbol{a}$. 76

Figure 4.6.1: a] [top] unfiltered $\Delta R / R$ data is $5 \% P / P^{0}$ at a total flow rate of 400 sccm for 4 vapors of toluene (blue), dichloromethane (red), methanol (green) and acetonitrile (purple). b) [bottom] Filtered $\Delta R / R$ data using the threshold set with the intensity factor.

Figure 4.6.2: Filtered principal component data using the threshold set with the intensity factor. Data is $5 \% \mathrm{P} / \mathrm{P}^{0}$ at a total flow rate of $400 \mathrm{sccm}$ for 4 vapors of toluene (blue), dichloromethane (red), methanol (green) and acetonitrile (purple).

Figure 4.6.3: Filtered normalized principal component data using the threshold set with the intensity factor. Data is $5 \% \mathrm{P} / \mathrm{P}^{0}$ at a total flow rate of $400 \mathrm{sccm}$ for 4 vapors of toluene (blue), dichloromethane (red), methanol (green) and acetonitrile (purple).

Figure 5.1.1: Sensor array was exposed to $5.0 \mathrm{P} / \mathrm{P}^{0}$ in $20 \mathrm{~min}$ on/off of toluene(blue), dichloromethane(red), methanol(green) acetonitrile(purple) and no analyte(grey) using ANN and a sparse autoencoder. Opacity is represents the sum of responses where stronger measurements and increased point density are more visible.

Figure 5.1.2: Sensor array exposed to toluene(blue), dichloromethane(red), methanol(green), acetonitrile(purple) and nitrogen(black) with increasing stepped saturated concentration responses of $0.5,1.0,1.5,2.0,2.5,3.0,5.0,10.0$ and $20.0 \% \mathrm{P} / \mathrm{P}^{0}$. A scatter plot is shown wherein the opacity points spotted is a sum of the responses elicited by the sensor.

Figure 5.1.3: Sensor array was exposed to 5.0 P/P in 20 min on/off of toluene(blue), dichloromethane(red), methanol(green), acetonitrile(purple) and nitrogen(black) using ANN and with a supervised method. The weights were initially seeded using the sparse autoencoder. A scatter plot is shown wherein the opacity of points plotted is a function of the sum of responses elicited by the sensor. This has the impact of making stronger measurements more visible, and also provides a stronger sense of point density in the projected space. 
Figure 5.2.1: Figure 5.2.1: Filtered $\Delta R / R$ data for the real-time analysis of 4 vapors (toluene, dichlorormethane (DCM), methanol and acetonitrile) over one hour with a measurement every five seconds. A $1 \%$ threshold response (difference between the maximum and minimum of the intensity factor) was applied to the $\Delta R / R$ data .............88

Figure 5.2.2: a) [top] PCA for the real-time analysis of four vapors within the open air of the laboratory for toluene (blue), dichloromethane (red), methanol (green), acetonitrile (purple) and nitrogen (black). Flow rate is uncontrolled as well as the concentrations of the analytes. b) [bottom] The enhanced image of the central cluster.............................90

Figure 5.2.3: a) [top] $P C A$ using the normalized $\triangle R / R$ for the sensor array in real-time analysis. b) [bottom] The Enhanced image for viewing of central cluster of points in the PCA of the real-time analysis..................................................................... 91 


\section{List of copyright materials}

Sela, L. \& Sobel, N. Human olfaction: a constant state of change-blindness. Exp. brain

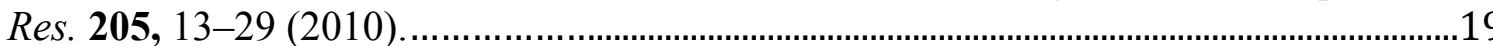

Ngiam, J., Koh, P., Chen, Z., Bhaskar, S. \& Ng, A. Sparse Filtering. Nips 1-9 (2011). at

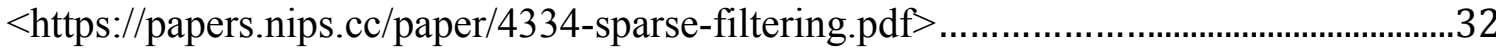




\section{Chapter 1: Introduction to Machine Olfaction}

\section{1 - Importance of Chemical Sensing}

Machine olfaction relies on pattern recognition of chemical sensor arrays; until recently, large amounts of data and low computing power hindered the advanced analysis of complex patterns and artificial intelligence. ${ }^{2}$ Machine sensing has grown in importance with the evolution of robotics and the drive to create humanoid robotics. ${ }^{3-5}$ This has come from both academic, industrial and domestic contributions towards the applicability and demand. ${ }^{2,6-8}$ Machine vision has led the field with the development of charge-coupleddevices (CCDs) and artificial intelligence-based pattern analysis algorithms. ${ }^{9}$

The introduction of the CCD chip in 1975, and its incorporation within cameras, enabled the first digital images to be taken. With digital photography came the manipulation of digital images, and the movement to more advanced imaging software, including facial recognition. ${ }^{4}$ Facial recognition technology has grown significantly over the last 20 years due to a several factors, including: an increased emphasis on research of neural network classifiers emphasizing real-time computation and adaptation, accessibility to hardware and an increased need for surveillance ${ }^{4}$

Support vector machines are a type of neural network classifier that was developed in the mid 90 's. ${ }^{10}$ This type of classifier minimizes the error in a set of vectors and uses a decision function to create the largest separation possible between separable classes; these networks were common in the initial analysis of digital facial recognition. ${ }^{10}$ Further investigations into the coding of mammalian facial recognition lead to the discovery and the development of sparse coding recognition. ${ }^{11}$ Additional developments came from the need to find less computationally expensive methods, as well as, the need 
to simplify the analysis. This can be done by reducing the tunable parameters. An example of such a method is sparse filtering ${ }^{12}$ (See section 1.3.4). These developments enabled the analysis of big data, with simpler and more efficient algorithms. The efficiency of the system is essential because the more data you have, the longer the length and larger the quantity of information that is required in order to train the network. The architecture, rate of learning, number of variables, number of layers and learning functions are just a few of the numerous factors which can affect the performance of Artificial Neural Networks (ANNs). ${ }^{13}$

As the CCD chip and ANNs advanced, the cameras became smaller, yet the quality and functionality of the camera was able to become more advanced. Within this work, there is a similar vision; this vision is to lay the understanding and foundation, while involving the temporal analysis of a chemical sensor array.

Current methods of analysis, within machine olfaction, focus on well-controlled, equilibrated sensor array responses. These responses use principal component analysis and linear discrimination analysis as the main pattern analysis method. ${ }^{14}$ Implementation of artificial intelligence within machine olfaction is a logical next step. While this is the obvious direction that needs to be taken, there are many challenges associated with the implementation of artificial intelligence. The challenges that need to be addressed include the fact that chemical interactions have many variables that need to be taken into consideration. ${ }^{14}$ Some of the variables that need to be addressed for the real-time analysis of vapors include drift, changing concentration and differing sensor/vapor time constants. Drift is the temporal shift in sensors' response that is due to environmental conditions. ${ }^{15}$ Within sensors this can be seen as a degradation of the sensors signal over time. 
Chemical sensor drift and degredation can be due to aging ${ }^{16}$, poisoning ${ }^{17,18}$, thermal and memory effects, changes in the environment ${ }^{19,20}$ and odor delivery system noise..$^{2,21,22}$ Statistical analysis, which is the main method of correction, uses the following methods to aid in the identification and correction of sensor drift: reference vapors, multivariate component correction, multivariate component deflation, filtering, attuning methods, independent component correction, orthogonal signal correction, adaptive methods, neural networks and evolutionary algorithms. ${ }^{21,23}$ As well, the development of sensors that are more resistant to sensor degradation can also assist in reducing sensor drift. This is, however typically a more expensive method. Depending on the statistical methods and the types of sensors used, concentration variation can affect the output of the sensor response due to sample to sample variation. ${ }^{24}$ Some sensors respond to concentration changes with a linear signal (tin oxide, composite sensors ${ }^{24}$ ), while others are non-linear (surface acoustic wave sensors ${ }^{25}$ ). Some methods of managing the concentration variance have been applied to sensor arrays. ${ }^{25-27}$ Unique to my work is the separation of the data into a concentration dependent variable and a concentration-independent pattern.

Correct categorization of vapors using carbon black - organic polymer composite sensors in uncontrolled environments must be able to handle variance, due to changes in rates of absorption Rates of absorption differ for each sensor, and also for each vapor exposed to the sensor, creating a large array of varying times. When performing real-time analysis, where identifying and quantifying a particular vapor are critical, time constants become an important component in the analysis. One time constant is defined as the time required for a particular sensor to reach $63.2 \%$ of its equilibrated response. ${ }^{28}$

Incorporation of these factors could contribute to earlier identification and quantification 
of vapors before equilibration has been reached. Some other methods for simultaneous quantification and identification have been demonstrated using exponential moving averages. $^{29}$

Little work has been done with chemical sensors to look at the temporal behavior in dynamic conditions. ${ }^{30}$ With many uncontrolled variables, when exposing sensors to realistic environments sensor performance is expected to decrease; this is due to the fact as the sensor responses need to be rapidly determined from a large pool of data, analyzed and categorized for the proper vapor.

As devices continue to shrink in size, and computer power is increased, these devices could one day be seen within smartphones and other electronics. Applications for this technology could include medical diagnostics, where chemical markers in breath can signal liver problems or cancer. ${ }^{31,32}$ Correspondingly, environmental and industrial monitoring would help control exposure to toxic fumes, as well as quality control of food products. $^{30,33-40}$ 


\subsection{1 - Mammalian Olfaction}

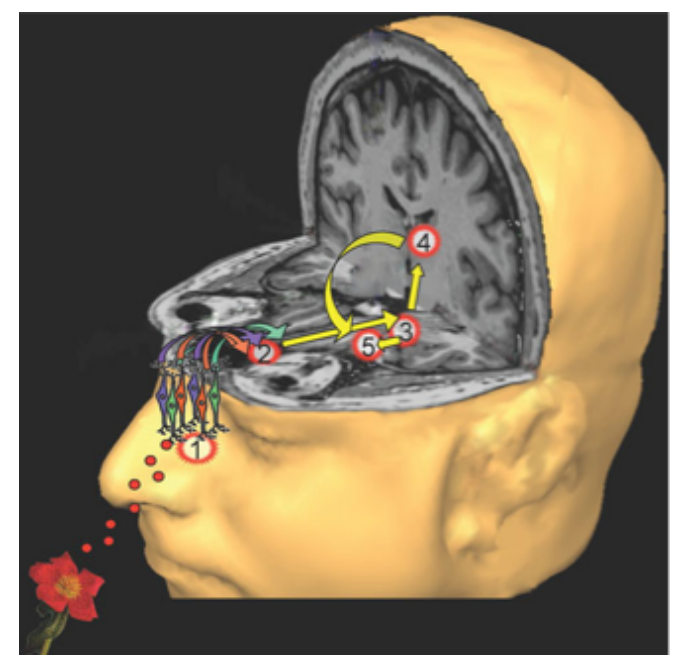

Figure 1.1.1: Human olfaction in the detection of vapors: 1) Olfactory epithelium 2) Olfactory bulb 3) Olfactory cortex 4) Thalamus 5) Orbitofrontal cortex. $^{41}$ (Copyright 2010 J. Exp. Brain. Res.)

Odor recognition starts with the olfactory sensory neurons, within the epithelium of the nose (Figure 1.1.1). Vapor molecules absorb onto the sensors causing a response that is transmitted, and gathered from the same sensor type within the olfactory bulb. Signals are then transmitted to the brain where the majority of pattern response processing happens within the olfactory cortex. Processing also happens throughout other components of the brain, but to a lesser extent. ${ }^{42}$ These include the higher cortical areas that assist with odor discrimination. As well as the deep limbic areas that are hypothesized to be also involved in emotional and physiological odor responses. ${ }^{42}$

There are approximately 12 million olfactory sensory neurons (OSN) and 400 different types present in the olfactory epithelium. ${ }^{42}$ Each OSN has a response to a particular odor or molecular species; these responses form a pattern that are compiled and 
organized within the olfactory bulb. The response of similar sensors is compiled into their respective glomeruli within the olfactory bulb. It is here that a spatiotemporal pattern is used for identity coding. ${ }^{41}$ The olfactory cortex is the core piece in decoding this pattern; it is to be retrieved from memory in order to form the vapors' identity, along with associated experiences and emotional responses. ${ }^{43}$ The main function of the olfactory cortex is to perform odor classification and differentiation. From the olfactory cortex, the information is then distributed throughout the brain to regions such as the orbitofrontal cortex, where experience-dependent modulation, effective coding and influences from other senses shape the consciously experienced signal. Other regions such as the thalamus also participate in odor identification, hedonic processing (pleasantness), olfactomotor control (sniffing), and olfactory attention. ${ }^{41,44}$

Odor identification has played an important role in human evolution with respect to communication, environmental monitoring and hunting/foraging. ${ }^{45}$ Although humans have a third of the receptors that mice have ( 388: 1200), the breadth of tuning suggests that humans may be capable of detecting almost as many odors; due to the lack of variants though, there may be a disadvantage in discriminating certain similar odors. ${ }^{45}$ Other factors affecting human olfaction include the emotional response. Emotional arousal has been shown to add to the quick identification of odors; pleasant and unpleasant odors were more often recognized than neutral odors. ${ }^{43}$ Emotional responses triggered by odors has been shown to increase odor recognition and the context in which the odor was previously recognized. ${ }^{43}$ This suggests that olfactory memory is closely linked to episodic retrieval. Thus, when the smell of a flower is recognized, we will recall 
memories of a garden where we initially smelt the unique odor and, are more likely to accurately identify the odor if linked to an emotional response. ${ }^{43}$

Using the human olfactory system as a model, it may be possible to develop a sensing system to differentiate, identify and quantify odors present in the surrounding chemical background. Current research efforts have concentrated more on a well-defined flow system with steady-state responses for pattern recognition without temporal or concentration fluctuations. ${ }^{34,46-52}$ Development of a novel method for the temporal analysis of the sensing system it may be possible to separate quickly changing chemicals from a drifting chemical background.

\section{2 - Sensor Arrays}

There are many different types of available sensors that are for use in the detection of vapors. The choice depends mainly on the limitations of the system, availability, cost, and application. Other types of chemical sensors include metal oxide

based, fluorescent based, resistance (polymer) and frequency base sensors. ${ }^{53}$ All of these sensors are relatively low-power and do not require a vacuum, as is the case for mass spectrometry, or high pressures with a gas flow, as needed for gas chromatography. The low cost and low operating requirements are attractive features of these chemical sensor arrays and make them more portable and accessible. ${ }^{53}$ Of all the sensor types, the composite sensor of non-conducting polymer carbon black was chosen because of its relatively simple design, its low maintenance, its cost effectiveness, its relatively simple processing, easy accessible and the fact that it has been well characterized in the past. Each type of sensor has a different mechanism of interaction. If these mechanisms are 
used together this would yield great diversity among the sensor responses. Sensitivity, reproducibility, intensity and response time are characteristic of the sensor type used, as well as the vapor to be detected.

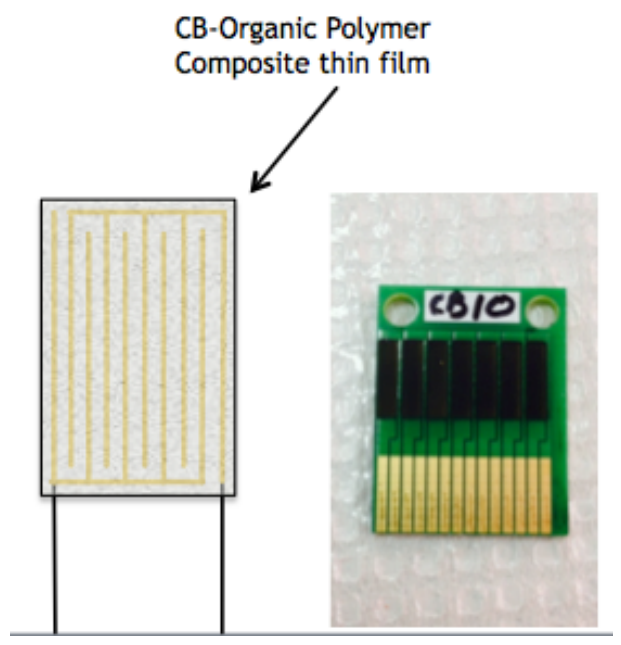

Figure 1.2.1: [Left] Gold plated interdigitated array with a film of carbon black non-conducting polymer film. [Right] Actual sensors used, size is $8 \mathrm{~mm}$ tall by 3 mm wide.

Interdigitated arrays (IDAs') are commonly used in resistive sensor applications ${ }^{54}$ (Figure 1.2.1). Resistance can be measured by deposition of the sensor matrix on the surface, by applying a small voltage and measuring the current that passes. As a vapor is passed across the surface of the sensor, some becomes absorbed and the resistance increases or decreases, depending. On what each vapor/sensor combination has a unique response. When used in an array a distinctive pattern much like a "fingerprint" is observed. Periodically applying this voltage gives a snapshot in time of the current state of the sensors. 
Increasing chemical diversity is essential for sensor performance to improve the performance of principal component analysis (PCA) by increasing the number of unique chemical interactions with each exposure. Therefore more information is captured by the sensor array as it is interacting with the analyte in a greater number of ways. Added diversity of the sensor array also has the added benefit of increasing the number of analytes that are detectable by having a wide selection of sensors. Additionally careful sensor choice can tailor the array to be more sensitive and respond faster to certain vapors.

For this work, resistive polymers were chosen because they are relatively quick to respond, cheap to produce, they can easily be made to be chemically diverse, and have been well characterized in the past. In particular Severin et $\mathrm{al}^{24}$ have used similar sensors in the development of analysis methods of vapors exploring some of the physical and chemical properties of the sensors relative to certain vapors.

Chemical diversity of the sensors adds to the number of possible vapors and to the variety of responses of the sensor array, increasing the resolving power needed for classification..$^{55}$ The resolution between analytes increases because there is additional information from each new sensor. This happens because resolution is typically measured using a form of linear discrimination analysis (LDA). The additional information from the sensors creates a larger matrix for larger dimensions within the analysis. Methods of diversification include: using chemically different polymers and doping of sensors ${ }^{56}$, different settings of the electrochemical potentials ${ }^{57}$ and different degrees of copolymerization $^{58}$. This is only for polymer-based sensors; there are other alternatives to polymer sensors that have their own applications; such as metal-oxide sensors, optical 
sensors, and gravimetric/acoustic sensors. Sensitivity, detection limits, and response times depend on the chemical makeup of the sensors and the method of detection. For this reason, one sensor type will be better than the other for a given analyte/application. The method of analysis, however, is relatively similar. Each sensor is set up in an array and from that array, a pattern unique to that chemical combination can be extracted, similar to that of a fingerprint. Using statistical methods like PCA, or similar methods (like linear discrimination analysis (LDA), etc..) similar "fingerprints" appear in similar places, depending on other variables such as noise.

\subsection{1-Polymers}

Non-conducting polymers consist of a series of repeating subunits that together make up a larger molecule. Different polymers are used within the sensor array as a means of increasing its chemical diversity. Increased diversity is useful for the larger number of dimensions to discriminate the complex responses occurring within the sensor array. ${ }^{59}$ Using PCA on such data, one would expect tighter clustering among similar responses and greater spacing between clusters with increased dimensionality. With increasing dimensionality the principal component space in which a cluster can appear is restricted, tightening the volume of the cluster.

The polymers used in this sensors array (Table 1.2.1) were chosen based on the characteristics of the functional groups such as alcohols, acids, phenols, oxides to name a few, providing a wide range of diversity for selecting a wide range of analytes. An additional consideration is that these sensors are well characterized within the steady state equilibrated responses due to previous studies and they are in general fast responding 
sensors. ${ }^{24,55,60-62}$ This provides a foundation to characterize the chosen sensors with further depth and complexity.

\begin{tabular}{ll} 
Non-conducting polymer & Acronym \\
\hline \hline poly(4-vinyl phenol) & PVP \\
poly(styrene-co-allyl alcohol) & PSA \\
poly(N-vinyl pyrrolidone) & PVB \\
poly(styrene-co-maleic anhydride) & PSMA \\
poly(sulfone) & PS \\
poly(methyl methalcrylate) & PMMA \\
poly(methyl vinyl ether-co-maleic anhydride) & PMVEMA \\
poly(ehtylene oxide) & PEO \\
\hline
\end{tabular}

Table 1.2.1: List of polymers used in carbon black-composite sensor array

\subsection{2 - Sensing Mechanisms}

The carbon black - organic polymer sensors are conductive because of the carbon black matrix surrounding the non-conducting polymer. ${ }^{24}$ The mechanism of sensing is absorption of the vapor analyte within the composite matrix causing swelling. ${ }^{56,63}$ The swelling of the composite sensor strains the conductive carbon black matrix, increasing the resistance of the sensor. Absorption of the vapor is dependent on the partition coefficient, $\mathrm{K}$, where $\mathrm{C}_{\mathrm{s}}$ is the absorbed vapor and $\mathrm{C}_{\mathrm{v}}$ is the analyte in the vapor phase. ${ }^{60}$

$$
\mathrm{K}=\mathrm{C}_{\mathrm{s}} / \mathrm{C}_{\mathrm{v}}
$$

The resistance of the sensor is proportional to the amount analyte absorbed into the sensor. $^{60}$

$$
\mathrm{R} \alpha \mathrm{C}_{\mathrm{s}}
$$

The measured changing resistance is dependent on the partition coefficient. The sensor is then linearly responsive to changes in concentration when $\mathrm{K}$ is constant. ${ }^{60}$ When 
the concentration of analyte is equal in the sorbent phase and vapor phase the sensor has reached equilibrium and will have the maximum response under constant temperature, vapor flow, pressure, and analyte partial pressure.

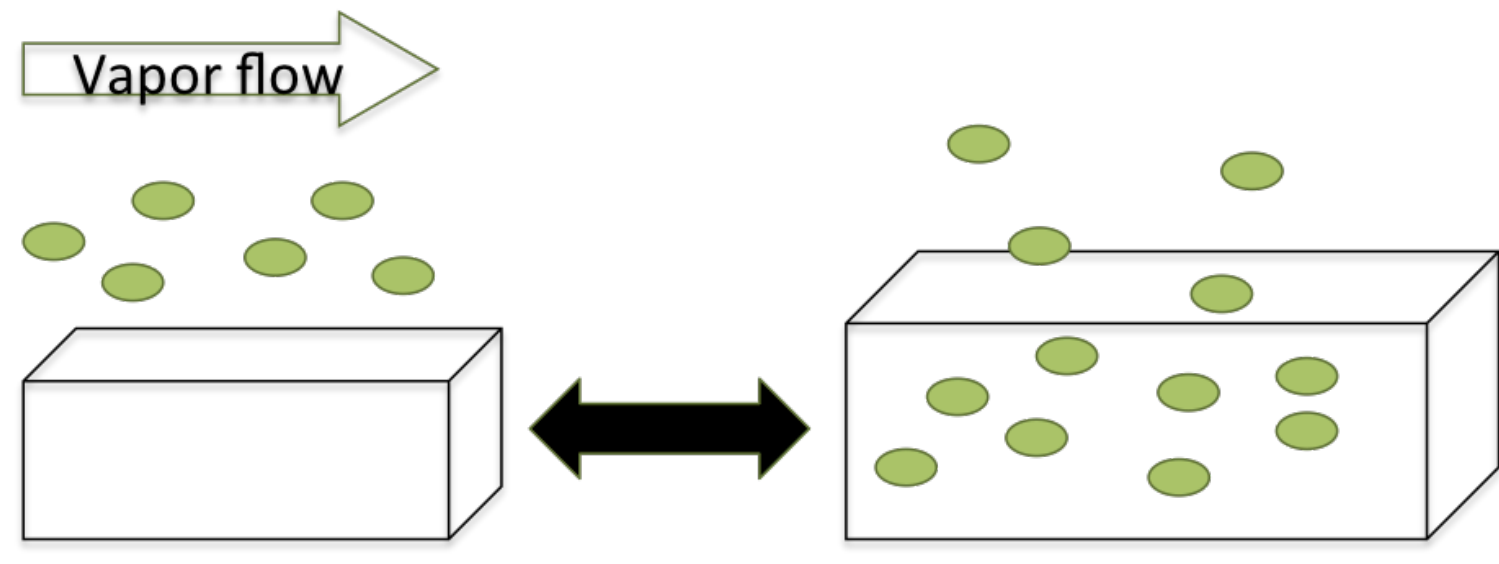

Figure (1.2.2): Reversible vapor (dots) absorption in carbon black-organic polymer composite sensors (box).

The resistance changes due to the increased separation in space between the conductive carbon black matrix are reversible. Once the analyte desorbs, the swelling decreases and the sensor relaxes back to its previous state (Figure 1.2.2). ${ }^{46}$ Relative concentrations of a vapor can be measured under identical conditions, where a standard curve is constructed for the sensor array because the composite sensors have been observed to increase in resistance with increasing partial pressure of the analyte. ${ }^{24}$ Quantification methods can be used to determine the nature of vapor as well as the quantity from a quick set of measurements for analyzed vapors with a calibrated sensor array. Using more than one vapor at the same time, the sensors have been shown to have an additive $\Delta R / R$ response. ${ }^{24}$ A sensor array of this type is capable of identifying complex mixtures of analytes. 
An additional consideration for analysis methods using a carbon black - organic polymer chemical sensor array for more realistic environments is that vapor concentrations are typically not uniform in the open atmosphere. There are variations within the sensor array response pattern, as a breeze will catch a vapor and carry it towards and away from the detector. ${ }^{30}$ If the sensor is fast responding, there could be noticeable fluctuations in response from small changes in vapor flow but the pattern should remain the same. However sensors with longer time constants might observe fewer fluctuations within this type of environment, smoothing out the response if it has sufficient exposure length is used to elicit a measurable response. ${ }^{28,64}$ The resulting pattern in this case will be different as the sensors try to remain equilibrated with the dynamic environments.

\section{3 - Data Analysis Methods}

\subsection{1 - Principal Component Analysis (PCA)}

Principal component analysis is used as a method of statistically separating the analyte response patterns. Pattern analysis is done by reducing the dimensionality within a complex multivariate data set, in this case, a matrix of the $\Delta R / R$ responses. The second step is to use a linear combination of variables to capture the most variance within the first component, and subsequent components within the newly formed orthogonal axis. However with increasing number of variables there are a greater number of components in PCA. This can dilute the variance by spreading it across more dimensions, but the variance should remain concentrated within the first principal component. 


\subsection{2 - Artificial Neural Networks (ANNs)}

The first ANN was developed in 1943, to mimic the thought process of a human within robotics. ${ }^{65}$ The mathematical description for the excitation "all-or-none" response of nerve cells is mimicked in the ANN. Nodes in a ANN use linear, radial basis function or sigmoidal curves to act as a threshold to decide whether an excitatory response should occur.

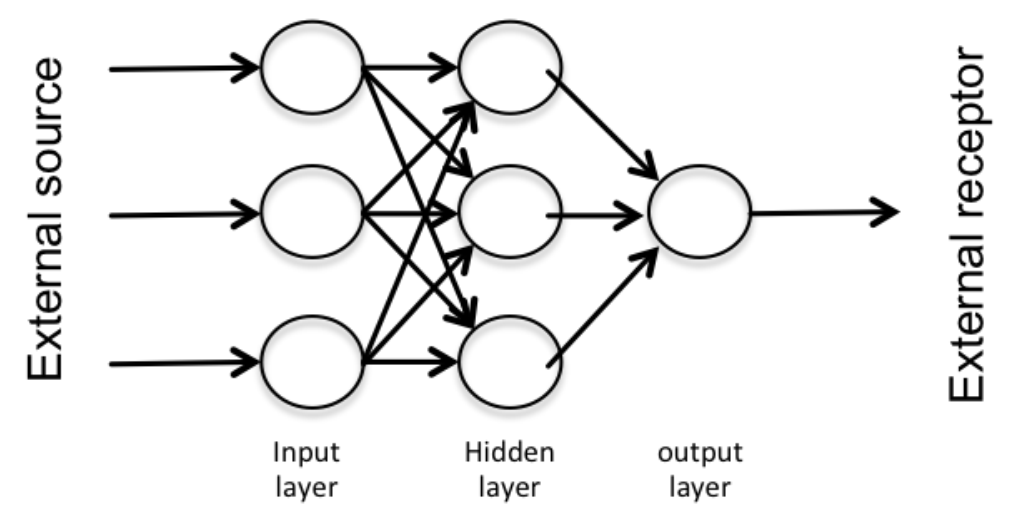

Figure 1.3.2: General ANN architecture

Building an interconnected network in a pyramid formation is the general layout for ANNs. The firing of one neuron will pass on the information to be manipulated by other neurons and firing will cause a similar process to occur until the last neurons are reached. At this point, the ANN will output the data for interpretation. This a classic ANN with an input layer (first nodes at the bottom of the pyramid), hidden layer (a number of nodes making up the body of the pyramid, (which is typically 3 , as it is sufficient for minimizing time and capable of handling the complexity for pattern recognition) and the output layer (last layer, top of the pyramid) which outputs the data ${ }^{13}$ (Figure 1.3.2). The more hidden layers present within the ANN the more complex the pattern recognition capabilities. ${ }^{7}$ 
Some parameters that are used to alter the performance of a ANN based on the needs are learning rate and momentum coefficient, transfer function, weight distribution factor, normalization of the input and output data sets and the number of hidden layers. ${ }^{13}$ Normalization of the network data is done to level the significance across all variables and place them on an even scale. The learning rate of the ANN controls the acceleration of change within the weights. Where the weight is the numerical representation of the strength between neurons, the higher the weight the more it contributes to the analysis and final output from the network. The momentum coefficient specifies how quickly the network gets out of local minima and higher learning rates train the network faster however this may be unstable. Slower learning rates take longer to train the ANN and are less likely to become unstable, however they may also become trapped within local minima unable to find optimization. ${ }^{13}$ The number of hidden layers within the ANN can be determined by using a series of combinations of the number of nodes and the number of hidden layers to find the best combination yielding the lowest error. The weight distribution factor is the initial values set for the weight matrix. There are a few ways to go about this such, as randomly assigning values to each weight, a uniform distribution, or a Gaussian distribution. Once determined the weights of the neural network are adjusted to provide the optimal correlations and categorization output from the data. ${ }^{13,66}$ Lastly there is the transfer function, this is a threshold placed on each neuron that will activate the node based on the sum of each weighted node. Transfer functions are non-linear and typically sigmoid, hyperbolic tangents or radial basis functions.

ANNs are adaptable, able to retain information in this way through training as well as over time through repeated exposures. As the network receives more information 
this will help improve its analysis, reducing the errors of prediction. This can be a problem if only small amounts of data are accessible as the network will not be properly trained and large variations within the results may be observed. ${ }^{7}$

\subsection{3 - Supervised \& Unsupervised Methods}

There are two main approaches to developing a method of statistical analysis using supervised or unsupervised techniques. A supervised learning technique uses additional information to instruct and guide the learning to know the right answer. The correct output is known and the weights are adjusted until the correct output is reached. The output consists of the classes associated with their numeric values. Examples of supervised learning are classification learning and regression. ${ }^{67}$ Classification learning focuses on separating data into categories based on the known associations. Regression analysis is to find hidden relationships. Support vector machines are a class of linear algorithm that maximally separate the classes of data using a hyperplane. A consideration when using supervised methods is the inherent bias that they add into the analysis. ${ }^{47}$

Unsupervised learning is a more autonomous method where the learning is done without a correct answer provided. In the unsupervised output, known answers are only required to identify and label the output neurons. The benefit of this technique is the learned structure of the data. ${ }^{67}$ Examples include reinforcement learning, clustering, associated rules and self-organizing maps. ${ }^{68}$

Semi-supervised learning is another type that blends the two methods by using the supervised knowledge together with information provided by the unsupervised sample

distribution information. ${ }^{69}$ Applications such as news filtering, e-mail spam detection, document classification, image classification all use semi supervised methods. 


\subsection{4 - Sparse Filtering}

Sparse filtering is an unsupervised feature learning method that is used to replicate the function of the intensity factor (see section 3.2) within an artificial neural network. ${ }^{12}$ The low number of tunable hyperparameters (number of features) allows for a simple and versatile feature-learning algorithm to scale well with larger data sets. The features for tuning include the population sparsity, lifetime sparsity, and high dispersal. Population sparsity is the large population of coding neurons with only some of the neurons active in response to a stimulus. ${ }^{70}$ Included within population sparsity is a different subset of neurons active within the population at any one time. This means that there is high population sparseness dispersed amongst the population of neurons. ${ }^{70}$ Lifetime sparseness refers to neurons which do not often (or rarely) produce a response. But when the response is produced it is a large response. This means that when a neuron does fire it carries with it more information with its responses. ${ }^{70}$ Because of the population dependence, when applied to unsupervised feature learning the sparse filtering method provides good approximations of the true data distribution.

Sparse filtering is calculated by first normalizing the feature distribution matrix by rows, then by columns and lastly summing up the absolute of all values. ${ }^{12}$ This sets each feature to be equally active within a $1_{2}$-norm (a vector norm) where a penalty for sparseness is applied. If the features are more similar, the more severe the penalty. (Figure 1.3.4) 

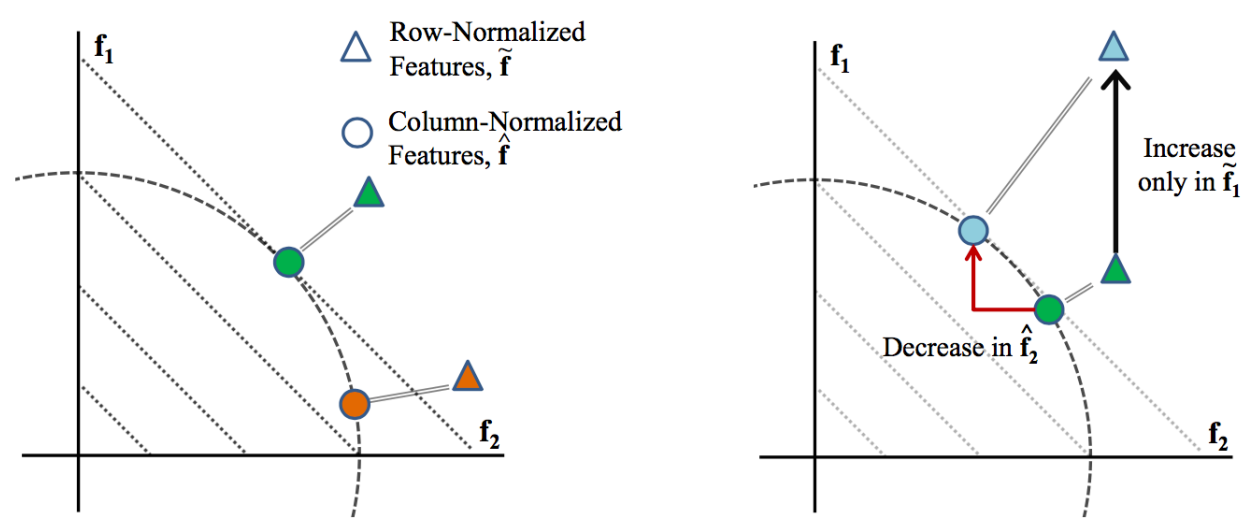

Figure 1.3.4: Left: Sparse filtering showing two features $(f 1, f 2)$ and two examples (red and green). Each example is first projected onto the l2-ball and then optimized for sparseness. The l2-ball is shown together with level sets of the l1-norm. Notice that the sparseness of the features (in the ll sense) is maximized when the examples are on the axes. Right: Competition between features due to normalization. We show one example where only fl is increased. Notice that even though only $f$ is increased, the normalized value of the second feature, $\hat{f}$ decreases. (Copyright 2011 Jiquan Ngiam et al.) 


\section{Chapter 2: Experimental Techniques}

\section{1 - Making Carbon Black - Organic Polymer Composite Sensors}

A sensor array consisting of eight chemically different sensors was constructed using PSA, PVP, PEO, PMMA, PSMA, PMVEMA, PS and PVB (see table 1.2.1). Composite sensors were made by adding $160 \mathrm{mg}$ one non-conducting polymer type dissolved using $20 \mathrm{~mL}$ of tetrahydrofuran. Then adding $40 \mathrm{mg}$ of carbon black (black pearls 2000 from Cabot corp) were added. The solution was then sonicated for 15 minutes. An interdigitated array (IDA) consisting of gold electrodes was cleaned in a solution of water and soap for 10 minutes, followed by sonicating for 10 minutes in ultrapure water (Milli-Q 18.2 $\mathrm{M} \Omega \mathrm{cm}$ at $25^{\circ} \mathrm{C}$ ) water, the array was then washed and sonicated in isopropanol for 15 minutes.

Deposition of the carbon black non-conducting polymer solution onto the IDA was done using an aluminum mask to airbrush a controlled and confined area over the gold electrodes. The deposition was determined to be complete when the IDA electrode surface was completely covered and the resistance was below $100 \mathrm{k} \Omega$.

\section{$2.2-$ Solvents}

Analytical grade toluene (Fisher Scientific Inc.), dichloromethane (Fisher Scientific Inc.), methanol (Fischer Scientific Inc.) and acetonitrile (EMD Chemicals) were used. Commercial grade nitrogen ( $>90 \%$ purity) from Praxair Canada was used as the carrier gas for all analytes. 


\section{3 - Initial Characterization of the Sensor Array}

An olfactometer was used as the controlled flow instrument, as well as a measurement device. Nitrogen is used as the carrier gas and its flow rate is regulated using mass flow controllers. There is a separate mass flow controller for each vapor. A standard glass bubbler was used for each vapor. This bubbler used a glass frit that helps disperse the carrier gas of nitrogen within each vapor. Electronic measurements were performed using a 34980A multifunction switch/measure unit from Agilent with a 3932A 80 channel reed multiplexer. Two point resistance measurements are taken every 2 minutes with a offset (B) of 0 , a max current of $50 \mathrm{~mA}$, direct current, Res of 5.5 and command execution times under 6 millisec for GPIB connection.

For repeated exposures of the analyte vapors as well as a method for randomly exposing different vapors to the sensor array, the total vapor flow of the system is consistently set to 400 standard cubic centimeters per minute ( $\mathrm{sccm}$ ). This is usually tested at $5 \% \mathrm{P} / \mathrm{P}^{0}$, as that concentration has been shown to respond well.

\section{4 - Temporal Analysis}

Within this work, a sensor array consisting of all 8 chemically unique sensors are used. Each sensor is exposed to the vapors using the olfactometer at $5 \% \mathrm{P} / \mathrm{P}^{0}$ for toluene, dichloromethane, methanol and acetonitrile. The $\Delta \mathrm{R} / \mathrm{R}$ at each point in time is used for the analysis, where $\Delta R,\left(R_{t}-R_{b}\right) . R_{t}$, is the maximum resistance at that point in time. Responses are normalized using the intensity factor. 


\section{5 - Dynamic Pattern Recognition}

A 62 sensor array was used consisting of PVP, PSA, PVB, PSMA, PS, PMMA, PMVEMA, PEO. Evaluation of the response to a gradually changing background by slow changes in the flow rate should let the sensor array stay equilibrated. Using toluene the concentration was gradually increased from a stable background in nitrogen to $10.0 \% \mathrm{P} / \mathrm{P}^{0}$, increasing by $0.25 \% \mathrm{P} / \mathrm{P}^{0}$ every 300 seconds. Flow rate for the slow gradual increase and decrease was held at $400 \mathrm{sccm}$. For another test the flow rate of nitrogen was held at $400 \mathrm{sccm}$ as the analyte vapor flow was slowly increased by $0.25 \% \mathrm{P} / \mathrm{P}^{0}$ every 300 seconds up to a maximum total flow rate of 420 sccm.

2.6 - ANN Analysis: Unsupervised Method

Linear transformations of the sensor data are done using the ANN and sparse filtering.

$$
f_{j}^{(i)}=w_{j}^{T} x^{(i)}
$$

Where $x^{(i)}$ represents the input pattern and $w_{j}$ is the weights associated with $j$.

Within sparse filtering, normalization is done across the sample population as well as the cell population, resulting in a normalized dispersion across the neurons.

$$
\begin{gathered}
\tilde{f}_{j}=f_{j} /\left\|f_{j}\right\|_{2} \\
\hat{f}_{j}=f^{(i)} /\left\|f^{(i)}\right\|_{2} \\
\operatorname{minimize} \sum_{i=1}^{N}\|\hat{f}\|_{1}
\end{gathered}
$$


After the linear transformation the neurons are all normalized. This is lifetime sparsity which reduces the number of nonzero elements within the matrix.

\section{7 - ANN: Supervised Method}

ANN training is done using a subset of the data from the full data set. Training is required for supervised learning but for unsupervised learning. The training is continuous until the network becomes stable. ${ }^{71}$ This is the first full set of exposures (one of each vapor 5 times). The purpose of training the ANN is to set the weights of each node close to that of the ideal. The training of a neural network can be adjusted using larger or smaller data sets, there is no set standard. However a larger training set will provide better results for the analysis, but requires more time.

Additionally for real time analysis, the optimization of the network can happen periodically, as a recalibration step based on how the sensors are performing. This may help with problems such as drift, sensor failure and with possibly the addition of new sensors.

Supervised ANNs within this work are done using a feed forward neural network. Consisting of 63 sensor values for 5 sequential time samples (315 dimensions). Two hidden layers of 18 and 6 neurons respectively follow, with one neuron at the output layer per category of vapor. All layers are fully connected to adjacent layers. Error is based on mean-square-error and Levenberg-Marquardt optimization is used in training the network. For visualization, activation at the output layer on the test data is reduced to 3 dimensions by PCA with the scatter plot showing values corresponding to the final 3 principal components. 
2.8-Real-time analysis

For real-time analysis, a seven sensor array was used. The non-conducting polymers used within the array include PSA, PVP, PEO, PMMA, PSMA, PS and PVB. Exposures were done within separate $50 \mathrm{~mL}$ beakers containing $5 \mathrm{~mL}$ of toluene, dichloromethane, methanol or acetonitrile. For a more dynamic environment, the lab air was used as the baseline vapor and once a stable baseline was achieved the analytes were presented to the sensor array one at time for a minimum of 5 minutes to a maximum of 10 minutes. Room temperature was monitored to be at $25.0^{\circ} \mathrm{C} \pm 1.0$.

Electronic measurements were performed using a 34970A Multifunction switch/measure unit from Agilent with two point resistance measurements taken every 2 minutes with a offset (B) of 0 , a maximum input current of $50 \mathrm{~mA}$, direct current. The number of power-line cycles (time during which an input measurement is samples) is 10 which takes approximately $167 \mathrm{~ms}$ for integration time to average out spurious signals from the DC measurements. Measurements are made by applying a known dc current to an unknown resistance and measuring the dc voltage drop. The attachment used to measure resistance is the 34902A 16-channel reed multiplexer. 


\section{Chapter 3: Characterization \& Quantification}

\section{1 - Normalized Resistance $(\Delta R / R)$}

Carbon black - organic polymer sensor arrays are known to have a response that scale linearly with concentration. ${ }^{24}$ The usefulness of this feature in a real world environment could be to monitor vapors and alert the user before they become harmful. ${ }^{30}$ This is beneficial in today's world, with rising integration of electronic devices around us, incorporated into our cell phones, clothing and skin. ${ }^{72,73}$ As these sensors become more available and less expensive to make, the quality and availability of applications would be expected to take a sharp increase, much like the camera did with the invention of cheaper electronics. ${ }^{74}$

The development of a gas sensor array that is capable of quantifying the vapor present within the atmosphere is not a new concept ${ }^{53}$; however, there is no system currently available that can identify and quantify multiple vapor species in real-time within the environment. Recent developments have shown promising results for the quantification of vapors in the steady state, or of a binary mixture of vapors using chemical sensors arrays. ${ }^{26}$ What is proposed here is a method in which the simultaneous quantification and identification of vapors can take place in real-time. 


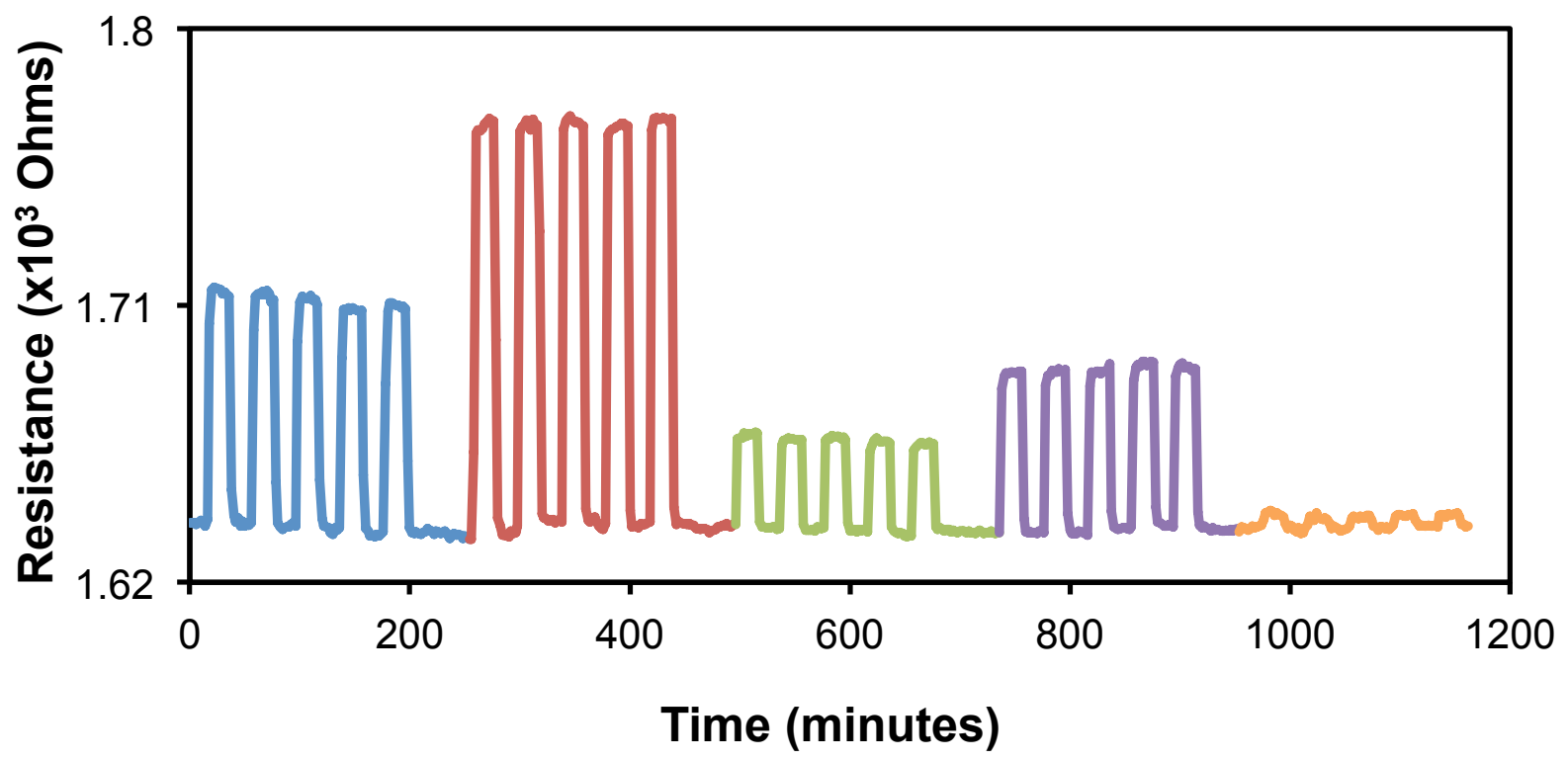

Figure 3.1.0: Temporal resistance response of Carbon black-Poly(ethylene oxide) sensor to toluene(blue), dichloromethane (red), methanol (green), acetonitrile (purple) and water (orange) over 20min on/off intervals. Data recorded at $25.0 \pm 0.2{ }^{\circ} \mathrm{C}, 400 \mathrm{sccm}$ nitrogen and 5 vol\% analyte pressure.

Exposures of the sensor array are done using 20 minutes on/off exposures to the analyte, using nitrogen as a background/carrier gas. (Figure 3.1.0) Each sensor is tested in controlled standard conditions using an olfactometer. The changing resistance is monitored and is different for each sensor and vapor combination. When exposing the sensor array to a vapor, a reproducible pattern is produced from the range of responses of the individual sensors.

There are two challenges for characterizing this sensor array, which include analyzing the quantification capabilities of the sensor array as well as the temporal responses. An additional factor is background drift, one example is when the response is tested using repeated exposures and the baseline response can gradually increase with each exposure to the same vapor for some of the sensors. This drifting causes additional variation within the analysis that needs to be accounted in some manner (which is 
addressed to some degree in section 4.6). Another similar problem occurs when there is a transition between vapors being exposed. Though the sensor array is equilibrated with nitrogen, there will be similar fluctuations in the baseline resistance of the sensor. These issues can cause a miss-classification of the vapor due to the resistance appearing to change either too little or too much. Due to the effect that one vapor has on the resistance, there may be an effect on the next vapors' resistance response in different combinations. The result is some sensors may be affected by the order in which they are exposed to the analytes. For the following experiments of this thesis, some were done using random exposures to attempt to circumvent this problem. On certain occasions, repeated exposures were used. The main difference between these results is that the random exposures show slightly larger $\Delta \mathrm{R} / \mathrm{R}$ standard deviations but the PCA results show a similar classification of the analytes. ${ }^{57}$

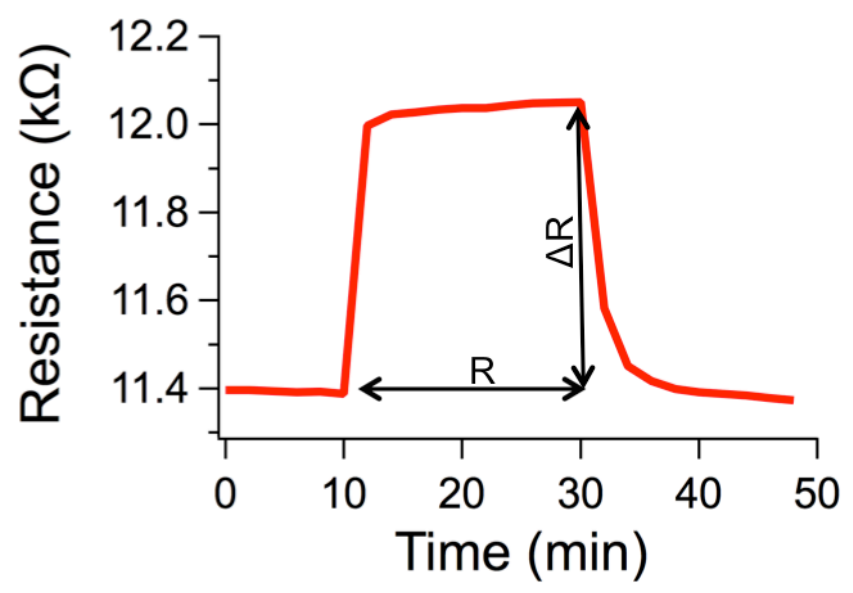

Figure 3.1.1: How $\Delta R / R$ is measured using standard methods. The baseline resistance is represented as $R$, the equilibrated peak response is the maximum at the end of exposure then subtracting the baseline resistance $(\Delta R)$.

Resistance-based chemical sensor responses have been found to scale according to the concentration of vapor present within the surrounding environment. ${ }^{24,26}$ As sensor 
array is exposed to gradually increasing concentrations of each vapor, of the resistance response increases accordingly. The method of detection is based on the absorption of the vapor into the chemical sensor, the rate of which is dependent on the makeup of each chemical sensor. ${ }^{56,60,75}$

Each sensor swells reversibly with the analyte(s) introduced and the resulting change in resistance is measured as the change in resistance from the baseline to the equilibrated response $(\Delta R)$ divided by the baseline resistance (R). (Figure 3.1.1) This system works well in most cases to normalize for background variation, however, there are some situations where this method of analysis will give false readings, such as reducing the actual sensor response due to background drift. There are currently many attempts at developing a universal method of dealing with sensor drift; however, as of yet there is no 'one fits all' solution. With the advancements of ANNs, the ability to learn and adapt may be the best option to solve this problem. ${ }^{21-23,69,76-83}$

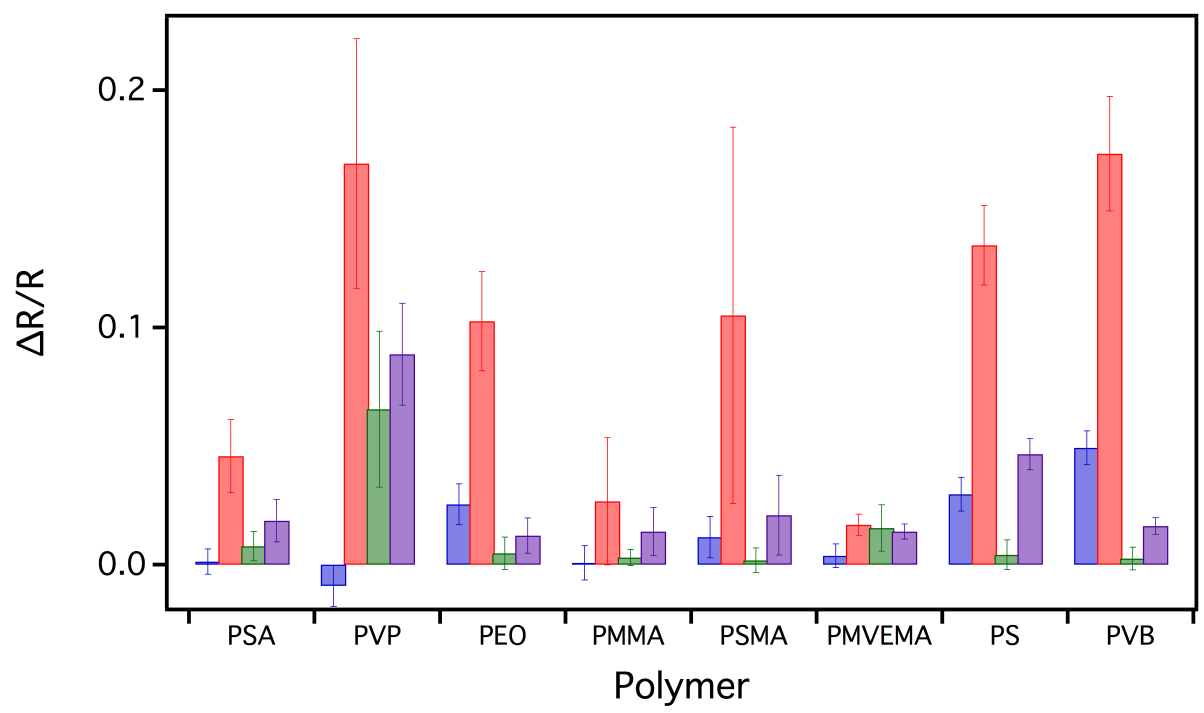

Figure 3.1.2: $\Delta R / R$ equilibrated response to vapors of toluene (blue), dichloromethane (red), methanol (green) and acetonitrile (purple). The average $\Delta R / R$ was taken over seven identical sensors with 5 exposures each. Exposures were random $20 \mathrm{~min}$ on/off exposures. 
Characterization of the sensor responses is done using 5 sequential 20 minutes on/off exposures at a total of $400 \mathrm{sccm}$ flow velocity to each vapor at $5 \% \mathrm{P} / \mathrm{P}^{0}$ with 60 minutes between changing vapors (Figure 3.1.2). Exposure to water showed very little change in the $\Delta R / R$ response of these sensors and, hovered around zero. Results from the PCA showed minimal variance and hovered around zero. Due to the lack of response of these sensors to water at $5 \% \mathrm{P} / \mathrm{P}^{0}$ they were left out of most of this analysis. However there is a slight response to water at $10.0 \% \mathrm{P} / \mathrm{P}^{0}$ indicating that the inclusion of water at higher concentrations will be necessary for the adaptation of this sensor array to a real world environment. ${ }^{20}$ (see Figure 3.3.0) The largest contribution to the observed variance between individual sensor responses was due to the noise within the sensors after equilibration had been reached. With a small $\Delta R / R$ for one sensor response, a small fluctuation in the equilibrated sensor response can cause large variations.

\section{$3.2-\triangle R / R$ PCA Characterization}

The sensor array response was tested looking for the clear distinction of the analytes, using the chosen composite sensors. Eight different sensors were tested with seven sensors for each type, for 55 sensors total. The one exception is for PSMA, of which one sensor did not produce a good response. Five exposures are repeated and it is expected that each exposure will produce similar patterns. When PCA is used to process the information, similar patterns should appear within the same principal component space, forming tight clusters. The larger the spread within the clusters, the greater the 
variance associated with that vapor. Therefore, the ideal case is tighter clusters with a larger separation between them.

Large separations between clusters are apparent after performing PCA on the equilibrated responses (Figure 3.2.0). Each vapor is showing a unique pattern that is significantly different from the others. Tight clustering within principal component space indicates that the sensor array response to each vapor is reproducible with small amounts of variation between the exposures.

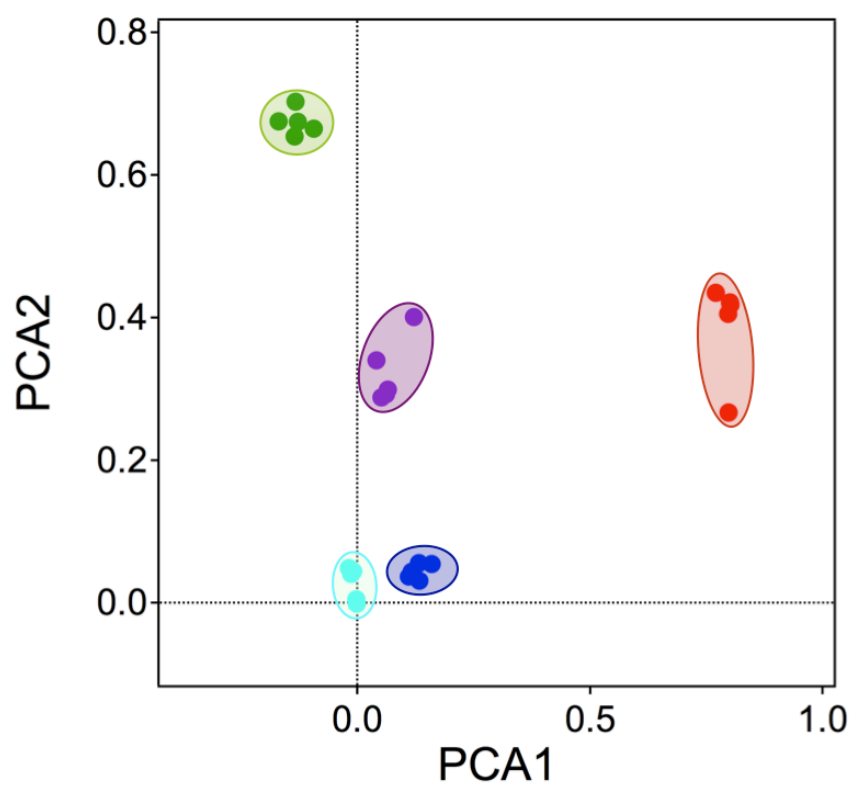

Figure 3.2.0: $P C A$ response using the sensor arrays equilibrated $\Delta R / R$ response of toluene (blue), dichloromethane (red), methanol (green), acetonitrile (purple) and water (cyan). The vapor pressure of each analyte is $5 \% P / P^{0}$ for 20 minute on/off exposures with 60 minutes separations between different vapors.

PCA initially included water within the analysis. But due to the very small, if any, $\Delta \mathrm{R} / \mathrm{R}$ response, water was not included in the determination of time constants. Water was also excluded from the PCA analysis for a better comparison of the principal components between experiments (Figure 3.2.1). 


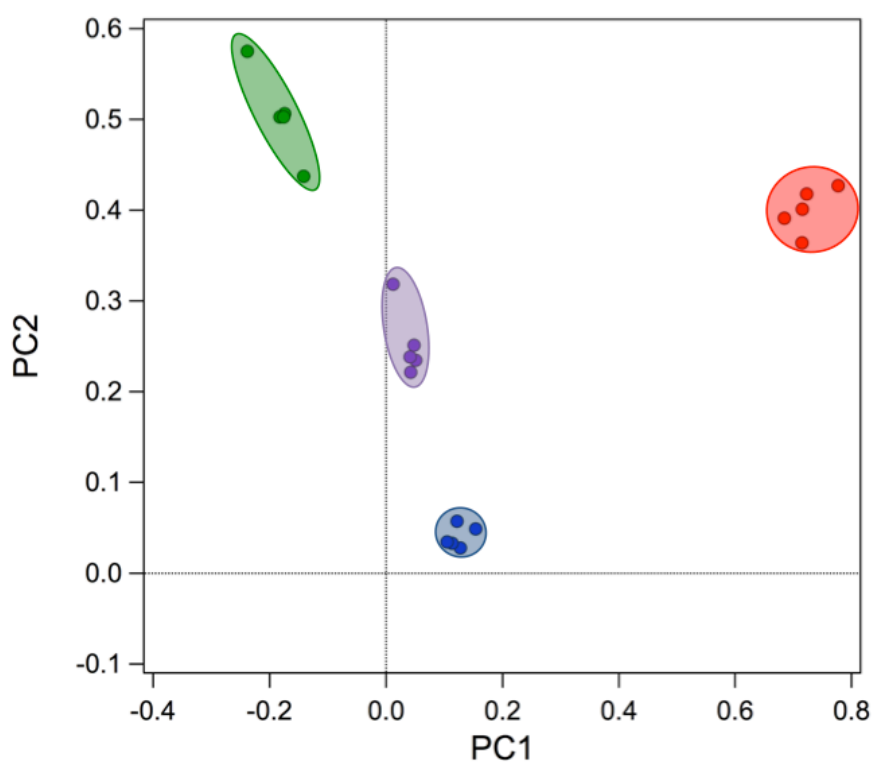

Figure 3.2.1: $P C A$ response using the sensor arrays equilibrated $\Delta R / R$ response of toluene (blue), dichloromethane (red), methanol (green), acetonitrile (purple). The vapor pressure of each analyte is $5 \% P / P^{0}$ for 20 minute on/off exposures with 60 minutes separations between different vapors.

\section{3 - Concentration Ramping and Intensity Factor}

To better understand the temporal performance of the sensor and associated drift, detailed characterization of the sensor performance is required. For quantification of vapor analytes, the important variable is the partial pressure of the analyte $\left(\mathrm{P} / \mathrm{P}^{0}\right)$ for analysis and not just the vapor pressure of the analyte. ${ }^{55}$ This means that the sensors are vulnerable to changes within temperature, volume and pressure for quantification of gas vapors. ${ }^{61,84}$ Understanding the thresholds for which the sensors become responsive, as well as the latency response time, are critical for use of sensor arrays in a realistic environment. These features as well as the concentration responses of the sensor array are examined by separating the intensity from the data. This creates a concentration 
independent pattern that will be used for classification as well as a concentration dependent variable called the intensity factor. Determinations of quantification limits were done under equilibrated conditions at constant temperature and flow rate in a controlled system to remove as much external variability as possible. Each sensor was allowed to come to equilibration by exposing the sensor array to each vapor for 20 minutes then off for 20 minutes (on/off exposures) for each analyte. In-between changing analytes, the sensor array was exposed to the nitrogen background for 60 minutes to eliminate any potential effects of a combined vapor response within the sensors. The nitrogen background flow rate was reduced in accord with the increase in flow rate of the analyte to maintain a vapor flow rate of $400 \mathrm{sccm}$.

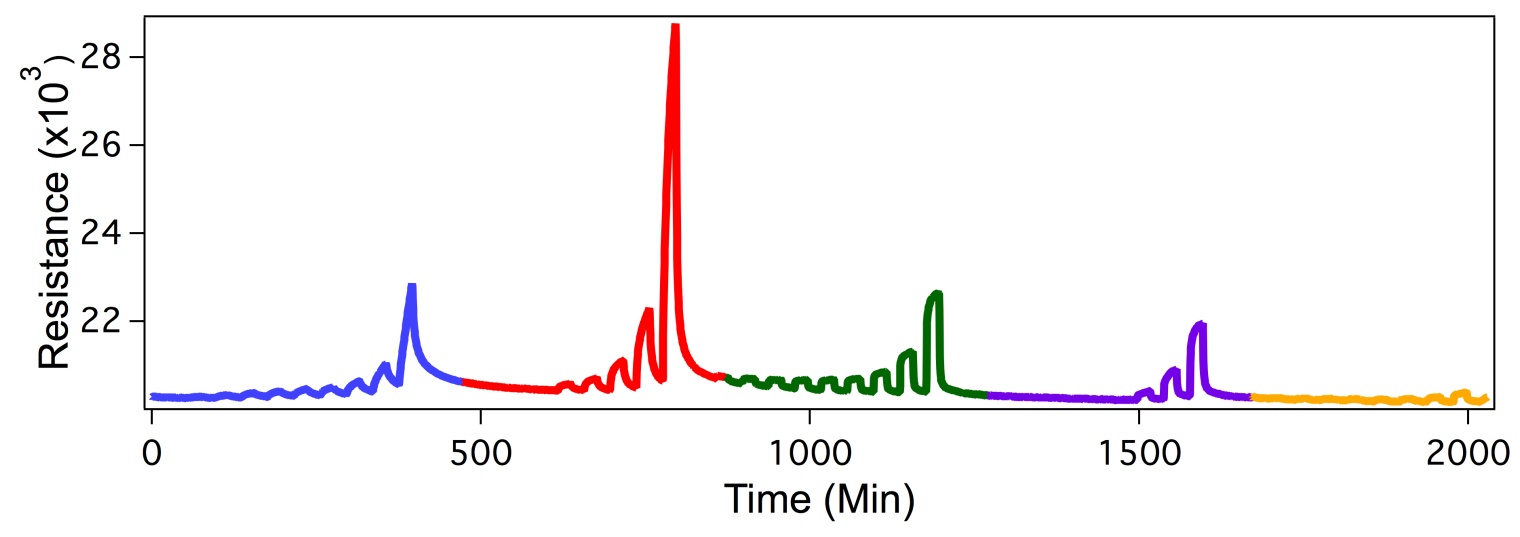

Figure 3.3.0: Temporal resistance response of Carbon black-Poly(vinyl butyral) sensor to toluene(blue), dichloromethane (red), methanol (green), acetonitrile (purple) over 20min on/off intervals. Data recorded at $25.0 \pm 0.2{ }^{\circ} \mathrm{C}, 400 \mathrm{sccm}$ nitrogen and $0.5,1.0,1.5,2.0,2.5,3.0,5.0,10.0$ and $20.0 \% P / P^{0}$ analyte pressure.

Initial observations show that some sensors have a minimal response, if any response, to some of the analytes until a particular concentration is reached. (Figure 3.3.0) When the vapor is turned off there is a small increase in the background resistance for most vapors, this is likely due to slow desorption of the vapor from the sensor before 
the next exposure is reached. Water can be seen to have a minimal response to this sensor at $5.0 \% \mathrm{P} / \mathrm{P}^{0}$ and below (Figure 3.2.0), but at a concentration of $10.0 \% \mathrm{P} / \mathrm{P}^{0}$ there is a very small peak that can begin to be observed. This raises some concern for humidity interacting with some of the sensors during measurements in the open atmosphere, causing some additional variation in the pattern response.

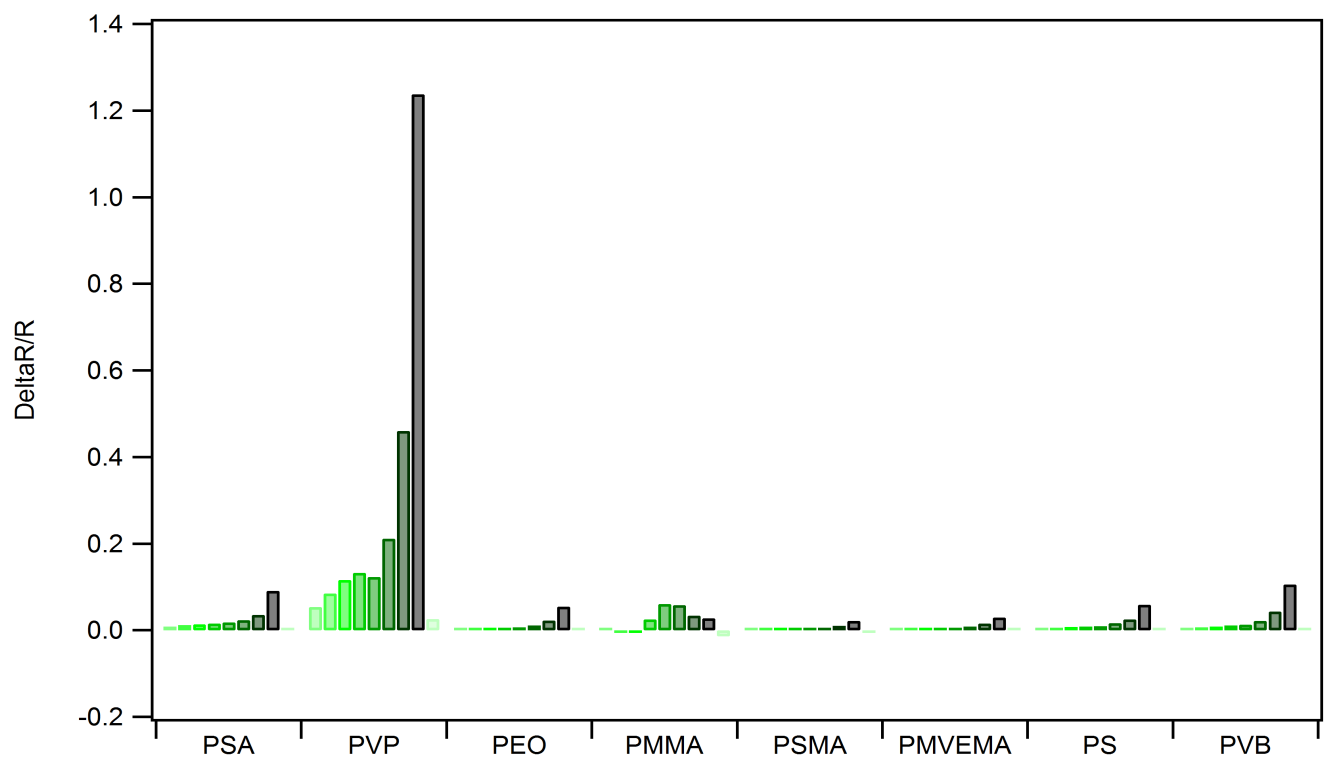

Figure 3.3.1: $\Delta R / R$ values for methanol with increasing concentration. Darker bars indicate increasing concentration corresponding to 0.5, 1.0, 1.5, 2.0, 2.5, 3.0, 5.0, 10.0 and $20.0 \% \mathrm{P} / \mathrm{P}^{0}$ respectively.

As was seen with every analyte tested, the resistance responses scale linearly with partial pressure. (Figure 3.3.1) Each sensor responded with greater sensitivity to a particular analyte. Therefore, an approximate threshold response can be observed, where below a certain concentration the sensor has random fluctuations around zero. Above this threshold, the sensor array responds in a linear and reproducible manner. The simplest method for removing the partial pressure component of the sensor array is to divide each $\Delta R / R$ response by the sum of the intensity, which we call the intensity factor (IF). 


$$
\text { Intensity Factor }(t)=\sum \Delta R / R \text { Vapor }(t)
$$

This method of normalization separates the data into its concentration independent component, where $\Delta \mathrm{R} / \mathrm{R}$ is the change in response of a specific sensor with respect to its baseline and $\sum \Delta \mathrm{R} / \mathrm{R}(\mathrm{t})$ is the sum of the response from each sensor within the array for the analyte at any point in time. This method allows the future use the intensity factor during real-time analysis to separate the data into these two respective components because, they are only dependent on the $\Delta R / R$ response of the sensor array.

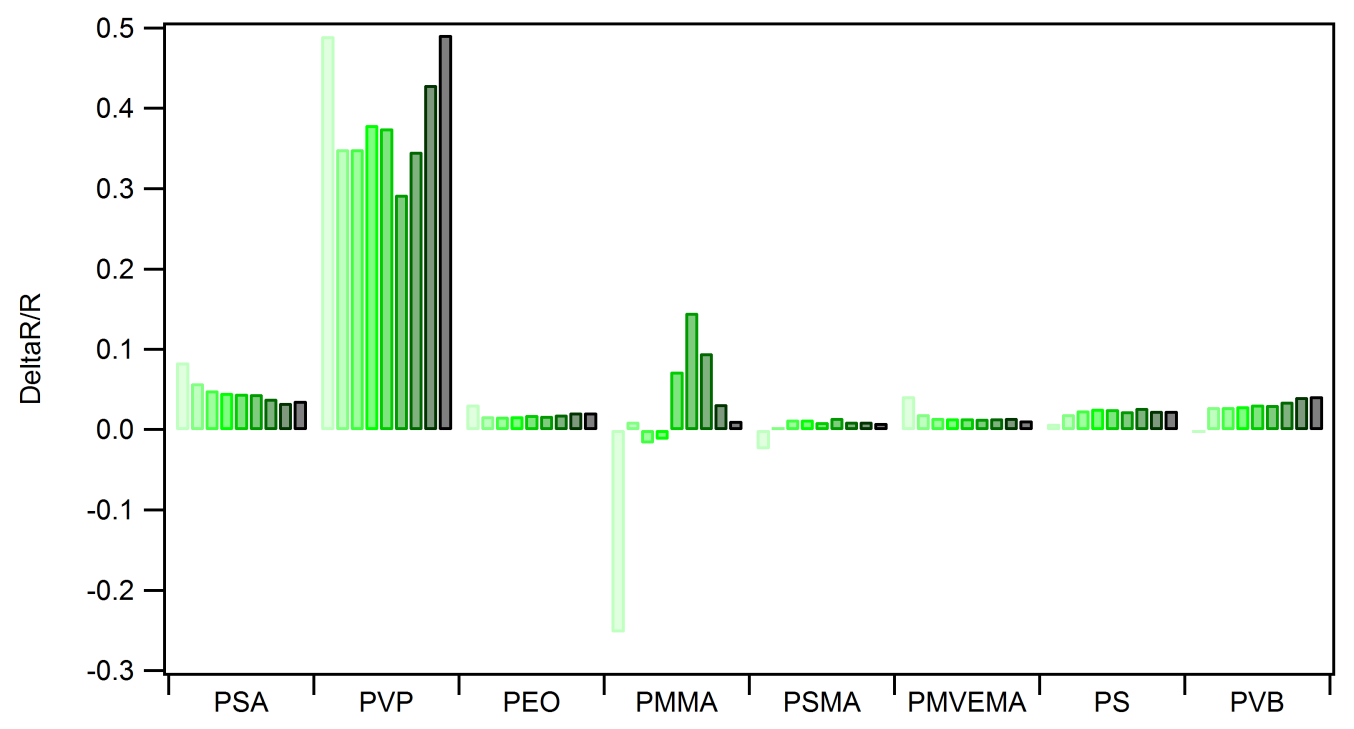

Figure 3.3.2: Concentration independent component is shown above after separation of the intensity factor from the original $\Delta R / R$ data. Preprocessed data from Figure 3.3.1.

The concentration independent component does not respond linearly, unlike the unprocessed principal components, remaining at a constant $\Delta \mathrm{R} / \mathrm{R}$ (Figure 3.3.2). Each sensor responds with the same intensity for each increase in concentration. Variance in the $\Delta \mathrm{R} / \mathrm{R}$ can be seen where the sensor is below the threshold or, as in the case of PMMA, does not respond well to methanol. A sensor that does not respond isn't a total 
loss as it does increase the selectivity of the sensor array for classifying particular analytes that may help when separating the patterns of complex mixtures. ${ }^{85}$ To handle similar problems in real-time analysis a filtering method has been demonstrated to limit the noise from a sensor array by only considering fast changing responses that pass a designated threshold in the PCA (see section 4.6).

\section{4 - Quantitative PCA Characterization}

PCA is used on the matrix of the equilibrated $\Delta R / R$ response for the sensor array, with each row being the $\Delta \mathrm{R} / \mathrm{R}$ with increasing vapor pressure of the analyte and each column a different sensor. As the concentration is increasing, the principal component also increases in a linear manner for concentrations tested (Figure 3.4.0). As the response increases, the points within principal component space expand outward, away from the center (approximately $(0,0,0))$. Weights used to calculate the principal components are determined from the averaged maximum equilibrated $\Delta \mathrm{R} / \mathrm{R}$ matrix for the $5 \% \mathrm{P} / \mathrm{P}^{0}$ data. Auto scale mean centering was not used with this method, because of the effects of concentration response. Even when using the same weights, the effect of concentration scaling would be altered within the principal component space by rescaling the data to fit about its mean. 


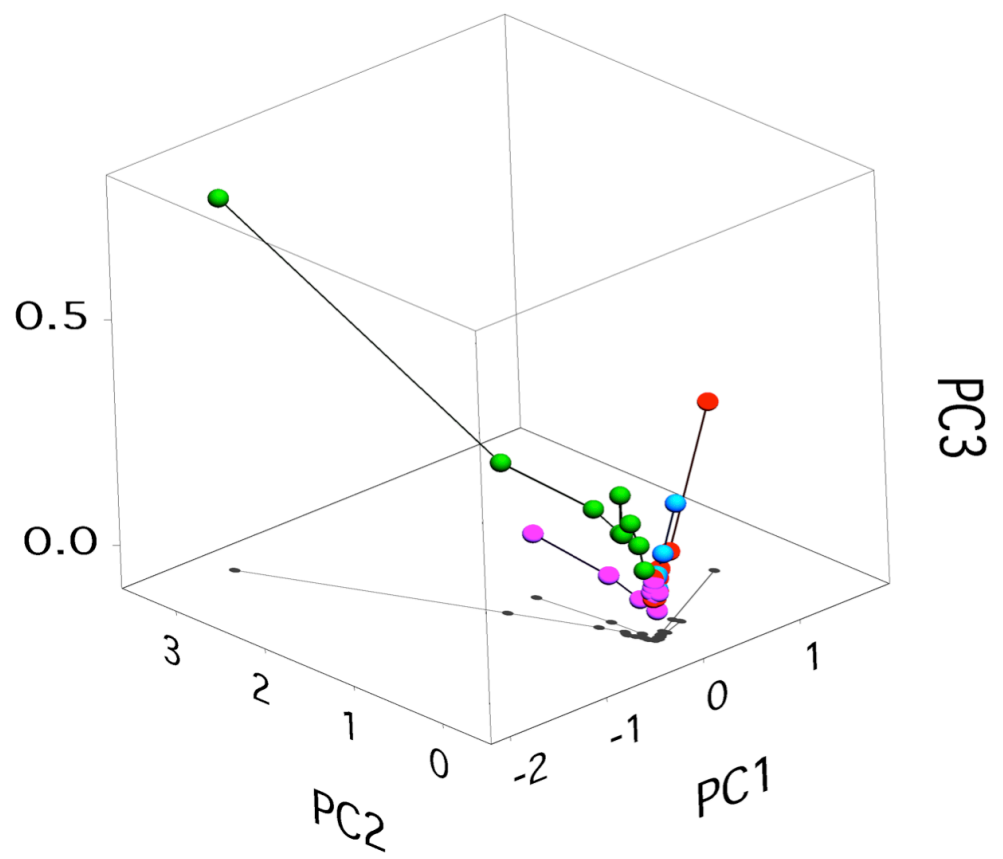

Figure 3.4.0: Principal component response of toluene (blue), dichloromethane (red), methanol (green) and acetonitrile (purple) with increasing concentration. Arrows show the direction of points with concentration increase of 0.5, 1.0, 1.5, $2.0,2.5,3.0,5.0,10.0$ and $20.0 \% \mathrm{P} / \mathrm{P}^{0}$ respectively.

The intensity factor has shown that the response of the sensors do scale linearly with increasing vapor concentration for the whole sensor array. The intensity factor separates the concentration component to maintain a consistent response for that vapor within principal component space. (Figure 3.4.1) At low concentrations, the noisy response from the sensor is visualized as random scatter. This is where a threshold can be set to identify a concentration limit where the sensor array will respond only when the intensity factor has reached a set response limit. 


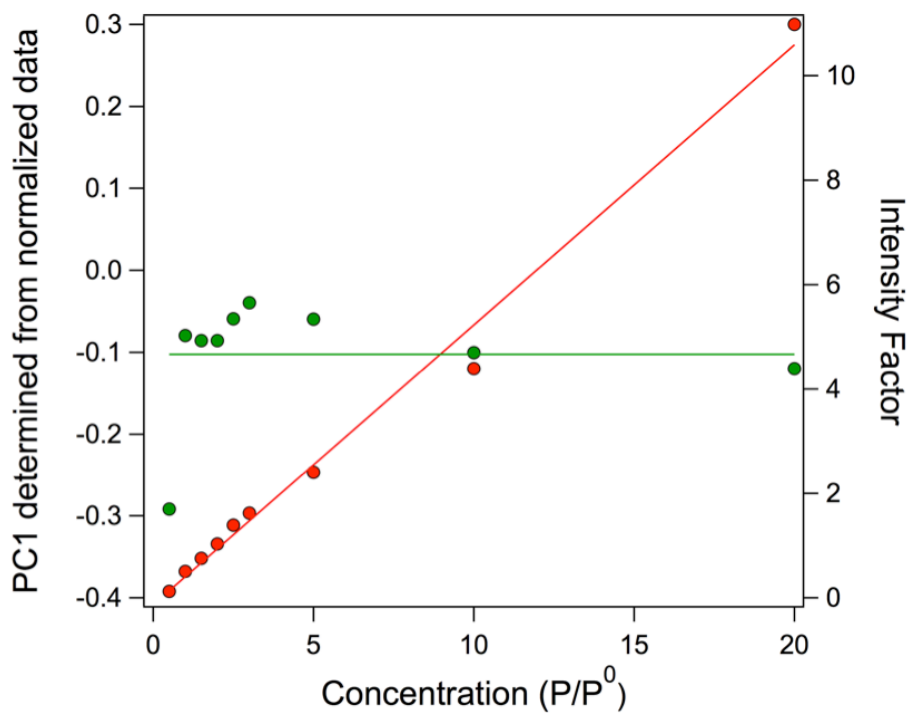

Figure 3.4.1: Normalized PC1 for methanol (Green) and intensity Factor (red) change with concentration. The green line represents the average response of PC1 determined for normalized data, and the intensity Factor (red) with an $R^{2}$ of 0.990. After the normalization of the response patterns, the increasing intensity factor as a function of increasing concentration, PC1 then becomes concentration independent.

Using the intensity factor, the $\Delta \mathrm{R} / \mathrm{R}$ matrix is normalized to separate out the concentration independent pattern (Figure 3.3.2). Normalized principal component values used the weights determined from the normalized and equilibrated $\Delta R / R$ matrix values at $5 \% \mathrm{P} / \mathrm{P}^{0}$. By separating the data to represent only the concentration independent component, similar patterns appear in similar places when the same weights are used within PCA (Figure 3.4.2). A similar identification method has been demonstrated within the olfactory sense of the zebra fish. They generalize along some dimensions of a coding space features like concentration and fine details such as identification of an odor along others. $^{86}$ 


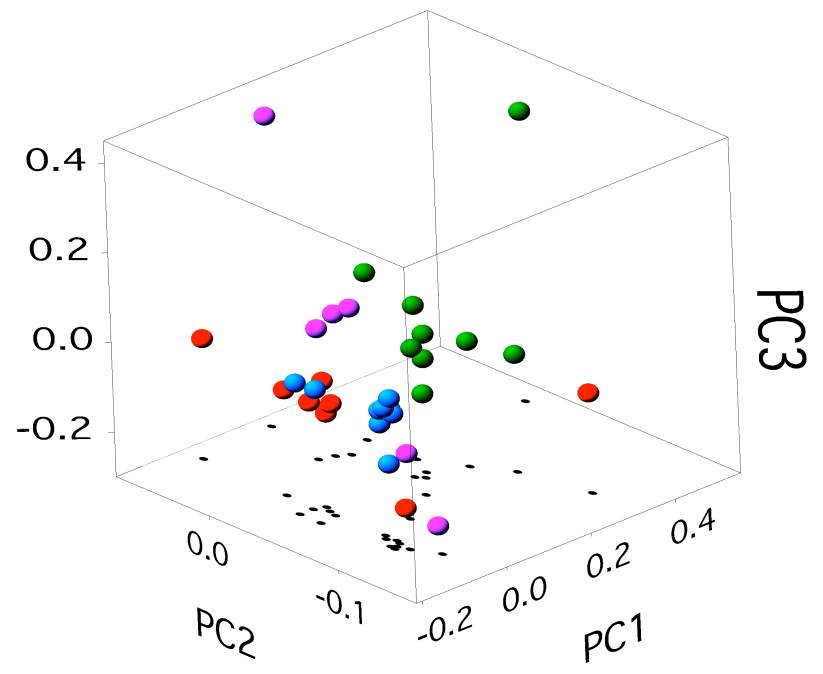

Figure 3.4.2: Principal component response of the concentration independent pattern for toluene (blue), dichloromethane (red), methanol (green), acetonitrile (blue). Preprocessed data are shown in Figure 3.4.0. Four points are outside the range of this graph and excluded because they contain only random noise fluctuations. These points are dichloromethane at $2.0 \% P / P^{0}(0.84,1.69,3.07)$, acetonitrile $1.0 \% P / P^{0}(-0.49,-0.51,-1.11)$, acetonitrile $1.5 \% P^{\prime} / P^{0}(-0.44,-0.85$, $-1.78)$, acetonitrile $3.0 \% P / P^{0}(-0.35,-0.28,-0.64)$

Characterization of the sensor array responses to the chosen analytes shows clear separation and tight clustering within the principal component space. Detection limits are determined by when the principal components become linear (Figure 3.4.0) and when the normalized principal component responses become consistent (Figure 3.4.2). The threshold for detection of each vapor is $0.8,11.8,4.0$, and 5.6 parts per thousand at $25 \mathrm{C}^{0}$ and $1 \mathrm{~atm}$ for toluene, dichloromethane, methanol and acetonitrile, respectively. For each vapor, a safe limit of detection is found to be $5 \% \mathrm{P} / \mathrm{P}^{0}$. Even though some vapors can be detected at lower concentration (for example methanol at $2.5 \% \mathrm{P} / \mathrm{P}^{0}$ ), it is still uncertain if the response is becoming linear at that point. A safe limit is defined in a manner that the response is very stable and linear. A $5 \% \mathrm{P} / \mathrm{P}^{0}$ corresponds to $1.7,19.7,7.9$, and 5.6 
ppt for toluene, dichloromethane, methanol and acetonitrile, respectively. As a comparison, the human olfactory systems is capable of detecting methanol at a concentration of $0.141 \mathrm{ppt}^{87}$ However evaluation of smells by the human olfactory system is a very complex system and can vary with many factors such as previous exposure, age, mood, gender, habits and environment. ${ }^{88}$

In response to more complex mixtures it can be expected that the formation of these response patterns and the observed trend of increasing $\Delta \mathrm{R} / \mathrm{R}$ with concentration, will hold even for different backgrounds. ${ }^{46}$ What might affect the $\Delta \mathrm{R} / \mathrm{R}$ however is a slowly changing background by a vapors slowly changing partial pressure in the background (see section 4.1). The order in which the different analytes are exposed to the sensor array may also alter their $\Delta \mathrm{R} / \mathrm{R}$, because of the alterations in the background resistance for some sensors. (Figure 3.1.0)

Identification of two or more vapors utilizing this method could also be possible. The intensity of the sensor response is linear to concentration and also previous studies have determined composite sensors to give additive signals to a mixture of vapors. ${ }^{24}$ The additive responses of two vapors would be expected to cause a fractional shift between the principal components based on the relative concentrations of each analyte. ${ }^{24}$ While using the same weights for PCA, the clusters within the principal component space would be monitored for changes in compositional analysis of the analyte vapor, while the intensity factor should be monitored for quantification.

Normalized response patterns at low concentration have increased scatter, which is due to a small $\Delta \mathrm{R} / \mathrm{R}$ divided by a small $\Sigma \Delta \mathrm{R} / \mathrm{R}$ for the array. This results in a larger number of the normalized $\Delta \mathrm{R} / \mathrm{R}$ and, in PCA, this appears as random scatter throughout 
the principal component space. Clustering of the analytes within the principal component space is more easily visualized by removal of the noisy and randomly scattered points for each vapor. (Figure 3.4.3) It is expected that using more broadly responsive sensors within the array will add to the resolution between the clusters. ${ }^{55}$ To improve sensor performance, additional sensors would be of benefit in distinguishing these vapors and may become necessary as the number of analytes increases. Mammals have a similar method that improves their ability to distinguish odors in a complex environment. It is to use many types of sensors (approximately 400) as well as many replicates of each type of sensors (12 million). ${ }^{42}$ The added replicates and types of sensors help to control for such fluctuations as humidity, temperature, temporal responses, and handling sensor failure. ${ }^{46}$

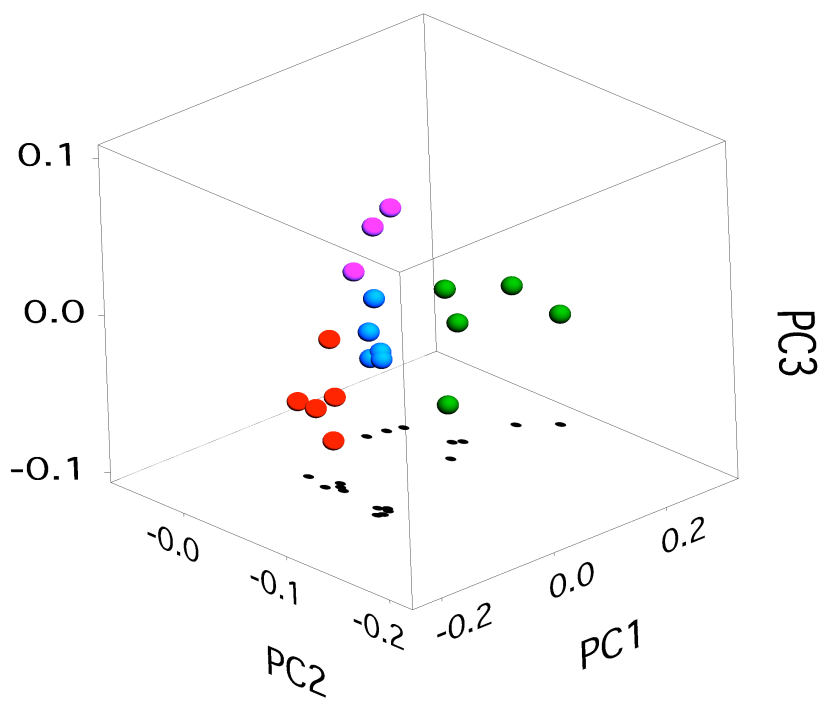

Figure: 3.4.3: PCA on the concentration independent patterns for toluene (blue), dichloromethane (red), methanol (green), acetonitrile (blue). Randomly scattered points below $2.5 \% \mathrm{P} / \mathrm{P}^{0}$ for toluene, dichloromethane, methanol and points below $5 \% \mathrm{P} / \mathrm{P}^{0}$ for acetonitrile have been omitted from the image. This is refined data from Figure 3.4.1. 


\section{$3.5-$ Sensitivity}

The sensitivity of the sensor array to a particular analyte is expressed as the slope of the intensity factor increasing with concentration. The higher the slope, the more sensitive the sensor is to that analyte. Changing the sensor types will alter the selectivity and sensitivity of the sensor array for the appropriate applications. This is much like the human nose, as it has adapted to be responsive to minute concentrations of $0.05 \mathrm{mM}$ for 4-hydroxyoctanoic acid lactone smells of coconut or $0.01 \mathrm{nM}$ for 2-isobutyl-3methoxypyrazine, which smells of bell pepper. ${ }^{89}$

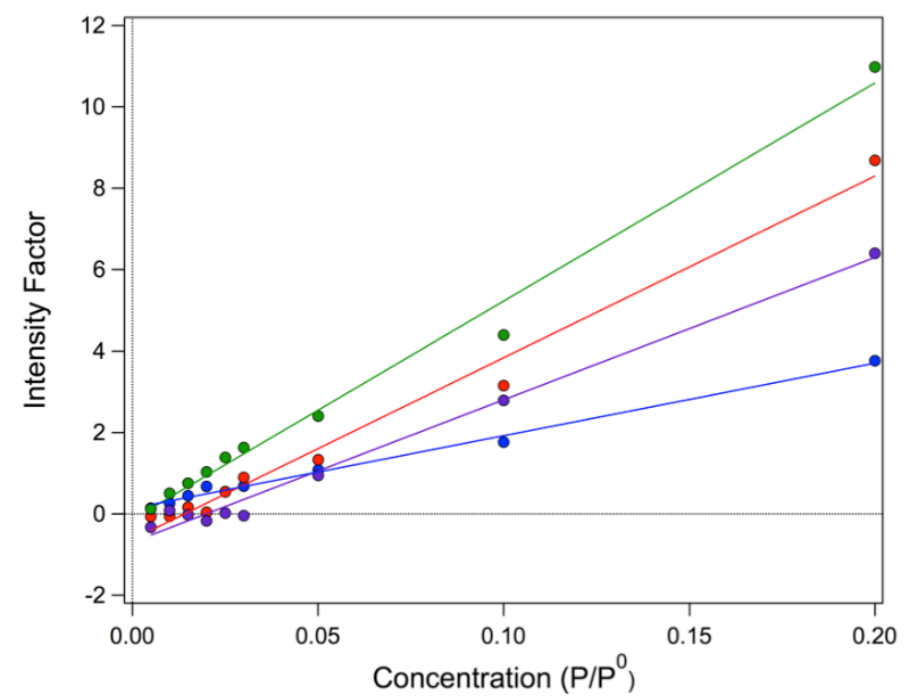

Figure 3.5.1: Intensity factor of the sensor array as a function of concentration toluene(blue), dichloromethane(red), methanol(green) and acetonitrile(purple). Each vapor has a correlation coefficient $>0.947$.

The chemical composition of this sensor array is mostly carbon black, a hydrophobic material that will dissolve in some organic solvents. This provides some basis for the sensitivity of the sensors to organic volatiles over other solvents types of solvents like water. Using the slope of the intensity factor, this sensor array is most 
sensitive to methanol, then dichloromethane, followed by acetonitrile, toluene, and then water.

The sensitivity of the sensor array is variable for each analyte. This sensor array is most sensitive to methanol, based on the slope of the intensity factor with increasing concentration. (Figure 3.5.1) Toluene is less sensitive than acetonitrile at lower concentrations. This is because initially at $3 \% \mathrm{P} / \mathrm{P}^{0}$ and lower, acetonitrile is not detected by the sensor array, and at $5 \% \mathrm{P} / \mathrm{P},{ }^{0}$ the intensity factor is roughly equal then surpassing it.

\section{6-Conclusions \& Future Work}

Analysis of the summed response of the sensor array provides an easier method than just $\Delta \mathrm{R} / \mathrm{R}$ or PCA for adaptation of the sensor array analysis into real-time monitoring. A threshold can be set using the intensity factor for monitoring the overall sensor array response to potentially filter out the background noise and drift from analyte peaks. (see section 4.6)

Application of this technique could be simple to add quantification features to potentially any sensor array. Required is the calibration of the sensor array to each analyte of interest and a linear response with increasing partial pressure. Non-linear responses may require more complex methods of analysis to attain similar results. Using an ANN is a possible technique to deal with more complex problems involving quantification. Using this method of analysis to separate the intensity factor and concentration independent pattern will help to easily classify the analytes and reduce the variance caused by concentration fluctuations within the environment. 
Future work includes utilizing the intensity factor by using more than one vapor. Separation of two vapors within a sensor array is possible and has been done before, however, not with the intensity factor method. ${ }^{26}$ Using multiple vapor (three or more) will be more of a challenge, and additional analysis methods may be necessary.

Additional measurements for full classification of the sensor array such as the recovery time, resolution, and further characterization of selectivity for this sensor array would be beneficial to get a complete picture of the sensor array response to individual as well multiple analytes. 


\section{Chapter 4: Temporal Analysis}

\section{1 - Time Constants}

Time constants of the sensors are calculated using a model to fit the experimental data. (Table 4.1.1) This model predicts the changing resistance over time, for the rising response of the sensors. ${ }^{28}$ An equation to model the recovery of the sensors is also described but is not covered within the scope of this work. Each sensor is analyzed independently for the rising time constants, where each time constant corresponds to $\sim 63.2 \%$ saturation.

\begin{tabular}{|l|c|c|c|c|c|c|c|c|}
\hline $\begin{array}{l}\text { Average } \\
\text { Response }\end{array}$ & PSA & PVP & PEO & PMMA & PSMA & PMVEMA & PS & PVB \\
\hline Toluene & $7.00 \pm 2.21$ & $5.05 \pm 3.38$ & $0.93 \pm 0.30$ & $6.76 \pm 5.16$ & $5.29 \pm 2.23$ & $3.55 \pm 2.02$ & $3.47 \pm 0.46$ & $9.78 \pm 1.04$ \\
\hline Dichloromethane & $4.12 \pm 1.85$ & $5.51 \pm 3.50$ & $0.92 \pm 0.19$ & $7.64 \pm 3.50$ & $2.60 \pm 1.10$ & $3.81 \pm 1.43$ & $2.47 \pm 0.45$ & $1.24 \pm 0.11$ \\
\hline Methanol & $1.77 \pm 0.96$ & $2.84 \pm 1.80$ & $0.69 \pm 0.22$ & $3.49 \pm 2.28$ & $2.09 \pm 2.29$ & $6.55 \pm 1.37$ & $1.24 \pm 0.47$ & $1.15 \pm 0.18$ \\
\hline Acetonitrile & $2.82 \pm 1.02$ & $5.25 \pm 2.71$ & $0.81 \pm 0.27$ & $6.34 \pm 3.22$ & $1.64 \pm 0.94$ & $5.09 \pm 0.91$ & $1.48 \pm 0.21$ & $2.05 \pm 0.20$ \\
\hline
\end{tabular}

Table 4.1.1: Average rising time constants for all carbon black-organic polymer sensors in minutes. Each sensor type consisted of 7 replicate sensors with 5 exposures each.

To control for other contributing effects that could alter the time constants the temperature was kept constant at $25.0 \mathrm{C}^{\circ} \pm 0.2 \mathrm{C}^{\circ}$ and the flow rate was held at 400 sccm. Both temperature and flow rate can contribute to the exposure-to-exposure variability their contributions are typically negligible compared to the $\Delta R / R$ sensor response and appears to be random. ${ }^{57}$ Additional variations for reaction time may be from the varying sensor-sensor thickness. ${ }^{62,84}$ The thickness of the sensors were not measured, but the best efforts at maintaining a consistent and reproducible preparation method were applied to all sensors by using a stencil and limit on the number of coatings applied to a sensor to attain conductivity across the IDA. 
The majority of the time constants $(62.5 \%$ of them) within this sensor array for the vapors used are below 4 minutes. (Figure 4.1.1) Higher time constants indicate that absorption is slower, the slowest being PVB exposed to toluene with a time of 9.78 minutes to reach $\sim 63.2 \%$ of its maximum equilibrated $\Delta \mathrm{R} / \mathrm{R}$. The fastest sensor observed is PEO exposed to methanol with a time of 0.69 minutes to reach $\sim 63.2 \%$ of its maximum equilibrated $\Delta \mathrm{R} / \mathrm{R}$. PVP is the fastest sensor overall, for all the vapors tested within this work.

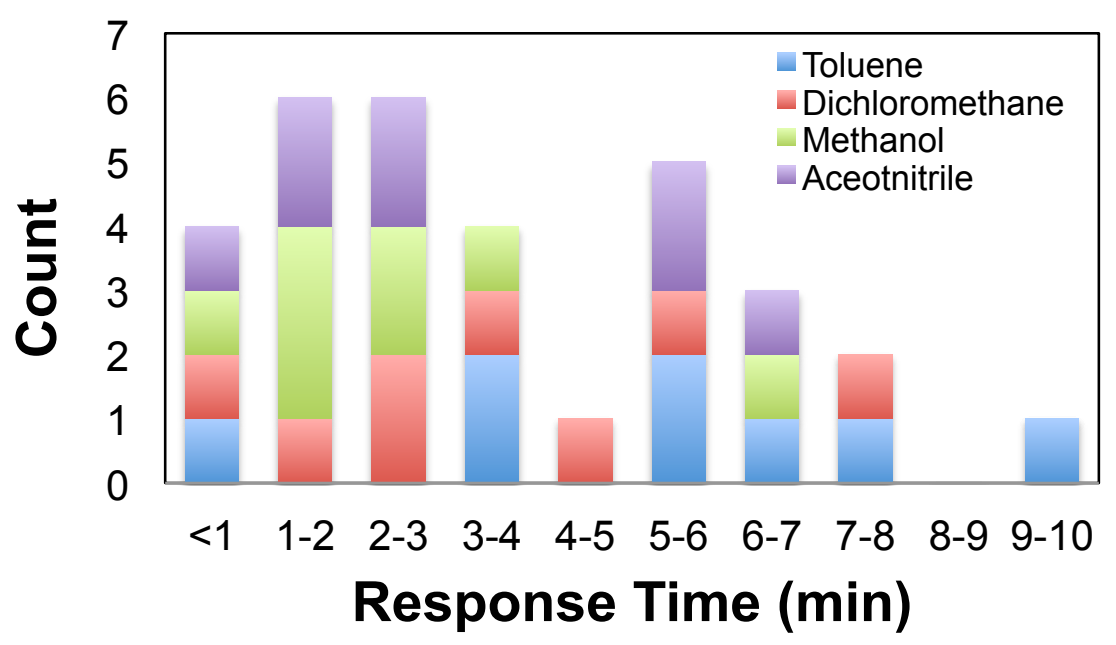

Figure 4.1.1: Distribution of response time within this sensor array for each vapor.Response time is determined from 1 time constant, which is the time to reach $\sim 63.2 \%$ of the saturated response.

Each vapor and sensor combination has a different time constants this is dependent on the combination of the two chosen. (Table 4.1.1) The variation of the sensor time constants can be quite extensive between sensors; an example of a large time difference would be 0.93 minutes to reach $\sim 63.2 \%$ saturation for PEO and methanol, as compared to 9.78 minutes for PMVEMA and methanol. As it can vary quite a lot within a sensor as well, looking at PSA the time constant jumps from 7.00 minutes for toluene to 1.77 minutes for methanol. (Table 4.1.1) With varying time constants, the equations used 
to calculate the theoretical resistance over time follow the trends of the experimental data. Vapor to vapor variation for the same sensor is typically on the order of a few minutes.

The exception to this observation is PEO, which has a variation in response time of 0.24 minutes. This is most likely due to the analytes chosen; with a greater variety of vapors it could be expected that a wider spread of time constants are present for all sensors.

The Pearson's chi-squared $\left(\mathrm{X}^{2}\right)$ test is used as a measure of fit between the experimental and theoretical results. (Table 4.1.2) The lower the $\mathrm{X}^{2}$ value, the better the fit of the theoretical model to the experimental data. ${ }^{90}$ Visual comparison of $\mathrm{X}^{2}$ values showed that the points deviated from the experimental model if the $\mathrm{X}^{2}$ value went above 0.3. This is well within the $\mathrm{X}^{2}{ }_{0.995}$ test statistic limit of 1.735 , meaning that within the experimental data the probability of getting a value more extreme than 1.735 is greater than $95 \%{ }^{91}$ There is no statistical difference between the experimental resistance over time and the theoretical temporal resistance.

\begin{tabular}{|l|c|c|c|c|c|c|c|c|}
\hline $\begin{array}{l}\text { Average } \\
\text { Response }\end{array}$ & PSA & PVP & PEO & PMMA & PSMA & PMVEMA & PS & PVB \\
\hline Toluene & 0.73 & 0.43 & 0.01 & 0.32 & 0.20 & 0.83 & 0.02 & 0.17 \\
\hline Dichloromethane & 0.14 & 0.56 & 0.11 & 0.26 & 0.24 & 0.19 & 0.18 & 0.11 \\
\hline Methanol & 0.06 & 0.29 & 0.06 & 0.58 & 0.66 & 0.08 & 0.05 & 0.02 \\
\hline Acetonitrile & 0.10 & 0.19 & 0.02 & 0.22 & 0.17 & 0.08 & 0.11 & 0.09 \\
\hline
\end{tabular}

Table 4.1.2: Calculated Pearson's chi-squared test values. $X^{2}$ values less than 0.3 are considered to provide a good fit of the theoretical simulation to the experimental data

\section{2 - Temporal Response}

Temporal $\Delta \mathrm{R} / \mathrm{R}$ changes of each sensor and the theoretical model show the lag in response between sensor types to the same vapor. (Figure 4.2.1) In the context of a sensor array with all the varying degrees of response the $\Delta R / R$ pattern becomes distorted. The 
pattern is not corrected until all sensors are equilibrated. (Figure 3.2.1) Of interest is the effect of such changes on the pattern and the resulting PCA response.

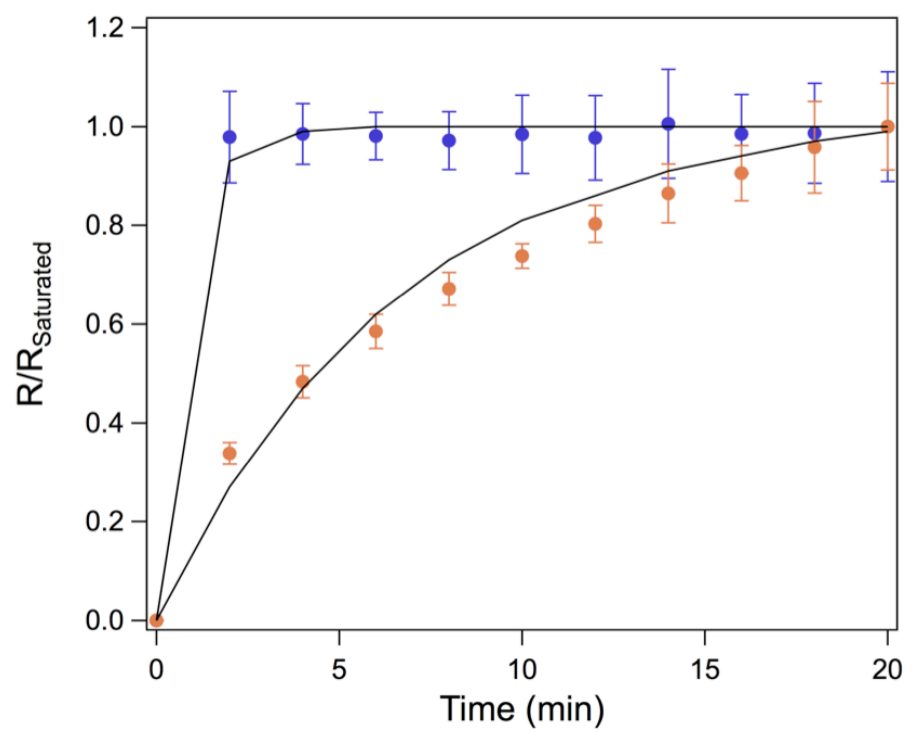

Figure 4.2.1: Comparison of rates between PEO (Blue), PMVEMA(Orange). Black lines represent the theoretical fit between the data points. Error bars represent the standard deviation of 5 exposures to methanol.

PCA was done using the weights calculated using the equilibrated $\Delta \mathrm{R} / \mathrm{R}$ response pattern. With the determined weights the principal components were calculated for each $\Delta R / R$ pattern every 2 minutes. The temporally changing $\Delta R / R$ patterns of the sensor array traces out the time-loops for the different vapors. (Figure 4.2.2) The time-loops all originate close to the $(0,0,0)$ point for the principal components as $\Delta R / R$ for each vapor is also hovering close to zero. As the $\Delta \mathrm{R} / \mathrm{R}$ begins to increase a pattern starts to emerge and the sensors with the faster time constants are the first to become equilibrated. This is likely the best explanation for the kinks observed within the time-loops. Much like the quantitative PCA characterization (Figure 3.4.0) the vapors extend outward from the origin towards their respective distributions within the principal component space. A realtime scenario using a similar method would make quantification extremely difficult 
unless the sensors are allowed to equilibrate. It is possible to follow a chemical trail using a ANN and fast responding sensors in a structured environment. ${ }^{85}$ With more analytes present within complex and dynamic environments, a more advanced sensor array and method of analysis will be required to obtain meaningful results.

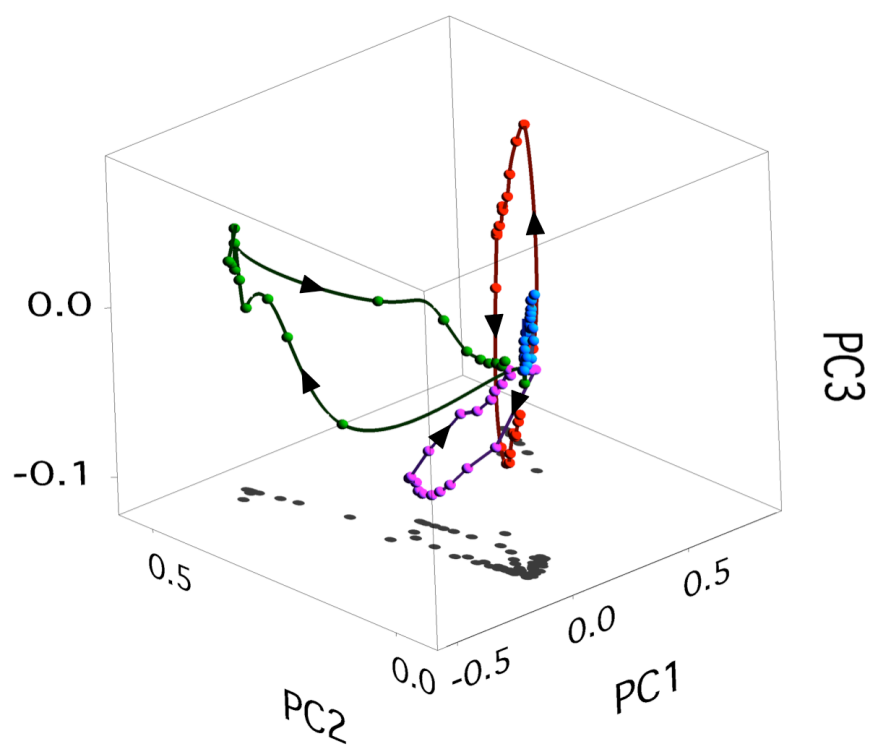

Figure 4.2.2: 3D plot of the averaged temporal response from the sensor array to the 4 vapors toluene(blue), dichloromethane(red), methanol(green) and acetonitrile(purple). Each point indicates 2 minutes up to the maximum $\Delta R / R$.

The temporally changing $\Delta \mathrm{R} / \mathrm{R}$ patterns of the sensor array are then normalized using the intensity factor to obtain the concentration independent pattern. (Figure 4.2.3) This removes the variance, causing the pattern to move and not settle until equilibrium is reached. The normalized response patterns for each of the four vapors are clustered within the principal component space. Random points are scattered throughout the principal component space, representing the $\Delta R / R$ patterns below the sensor array threshold response. To clearly see the clustering that is going on in principal component space and not the random scatter coming from the low responses below the sensor arrays 
threshold (Figure 3.5.1), randomly scattered points are cleared away (Figure 3.4.3).

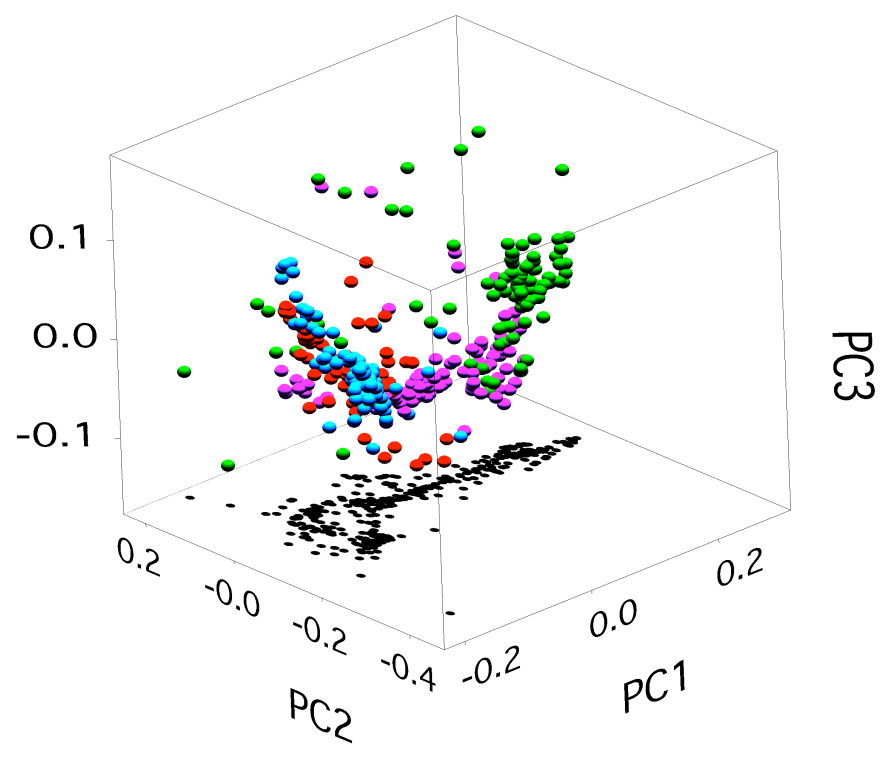

Figure 4.2.3: Normalized temporal response from Figure 4.2.2 using the Intensity Factor. Data points close to zero have been omitted from the image because they are randomly scattered within principal component space. The removed data points coincide with the first 4 minutes of analysis and the last 16 minutes after the equilibrated response.

Removal of the points below the threshold reveals the tight clustering and separation between vapor response patterns within principal component space. (Figure 4.2.4) A much clearer picture for the classification of the vapors is obtained demonstrating the potential use of a threshold function within real-time analysis. The point at which the principal components start to cluster is at the equilibrated response of the sensors. The sensor array data for repeated exposures (Figure 3.2.1) were used as the equilibrated standard. If the points fall within the cluster, the sensor array could likely identify the vapor using these methods under similar conditions. With this method the vapor clusters first appear and hold within the principal component space after 4 minutes 
of analysis. (Table 4.2.1) Clustering of all the points within the principal component space for each vapor becomes stable at 10 minutes after the exposure. (Table 4.2.1) That is the time for equilibration of all the sensors within the sensor array measured when the principal component points start to cluster. (Figure 4.2.3) Tailoring the chosen sensors to minimize the time constants for the analyte vapors would increase sensitivity and could further accelerate the classification and quantification process. ${ }^{29}$ Separation between the different analytes could further be improved (Figure 4.2.3) by using a filtering method to sort out the random noise. (Figure 4.2.4) Additional sensors with increased diversity would also be expected to add to the resolution of the analytes within the principal component space. $^{55}$

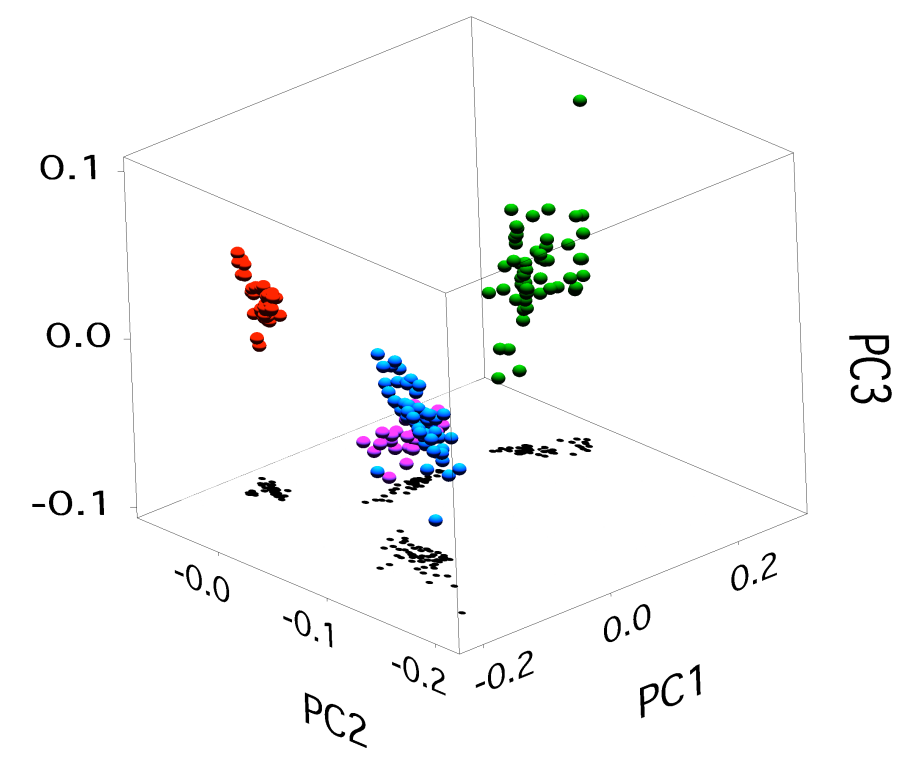

Figure 4.2.4: Normalized temporal response from Figure 4.2.2 using the Intensity Factor. Data points close to zero have been omitted from the image because they are randomly scattered within principal component space. 


\begin{tabular}{l|l|l}
\hline Vapor & PCA & PCA + IF \\
\hline \hline Toluene & $10 \mathrm{~min}$ & $2 \mathrm{~min}$ \\
\hline Dichloromethane & $8 \mathrm{~min}$ & $2 \mathrm{~min}$ \\
\hline Methanol & $8 \mathrm{~min}$ & $4 \mathrm{~min}$ \\
\hline Acetonitrile & $10 \mathrm{~min}$ & $4 \mathrm{~min}$
\end{tabular}

Table 4.2.1: Time before clustering within principal component space for PCA and normalized PCA $(P C A+I F)$.

\section{3 - Interactions}

Interaction within the sensor array can be measured from the intensity of response of an analyte for a sensor, the selectivity of an analyte for a particular sensor and the temporal response. In determining the different levels of interaction of an analyte, the sensor array should be diverse and large to capture many interactions. The advantage of carbon black - organic polymer composite sensors is that they can be made to increase the diversity of the sensor array. They have also shown the of being more diverse and having a larger resolution factor than a comparable tin oxide sensor array of equal size. ${ }^{46}$ A higher resolving sensor array is preferred because it can probe deeper into the different chemical properties brought about by the different chemical interactions within the sensor array. $^{55}$

Sensitivity and selectivity are controlled by the favorability of interactions between the analyte and the sensor through chemical and physical properties. ${ }^{75}$ Where strong interaction will produce larger signals but there will likely be more hysteresis, 
conversely weak interaction will have little hysteresis but weaker signals. ${ }^{75}$ Favoring absorption of the analyte is dependent on the chemical interactions which will be in favor of absorbing the analyte into the sensor, these interactions are called solubility factors. ${ }^{92}$ Solubility factors, affecting the sensors themselves, are selected based on the range of vapors that are fairly accessible within the environment and industry. The variety of patterns that can come from a given sensor array are extensive and demonstrated above. (Table 4.3.0) Ranges for each vapor cover a large portion of the spectrum, as defined by the five different solvation parameters. These parameters help define the contributing factors and effects of solvent - solute interactions, as well as solvent - solid phase interactions. PSA, for example, has separate repeating monomers with either a phenyl, or hydroxymethyl bonded to a carbon backbone through a $\mathrm{C}-\mathrm{C}$ bond. The fastest response time is to methanol, that is very similar to the hydroxymethyl group. This suggests that some hydrogen bonding occurring between the two molecules and, with methanol being small, there would presumably be low steric hindrance. Toluene is the slowest to reach equilibration with PSA; it is significantly larger than methanol with its own phenyl ring connected to a $-\mathrm{CH}_{3}$. Looking at the solvation parameters, PSA responds better to molecules with a lower $\mathrm{R}^{2}$, a lower $\mathrm{p} 2 \mathrm{H}$, a higher a2H, a higher $\mathrm{b} 2 \mathrm{H}$ and a lower $\log \mathrm{L}$ (these parameters are defined in Table 4.3.0). Similar functional groups within sensors would therefore be expected to respond with a similar pattern, but with different intensities and time constants. ${ }^{92,93}$ This raises the possibility of using these time constants within the analysis to obtain further information about the analytes under study. A much more versatile method of analysis, such as ANNs, will need to be used to account for large number of variables in real-time. 


\begin{tabular}{l|l|l|l|l|l|}
\hline Average Response & $\mathbf{R}^{\mathbf{2}}$ & $\mathbf{p 2 H}$ & $\mathbf{a 2 H}$ & $\mathbf{b 2 H}$ & $\mathbf{l o g L}$ \\
\hline Toluene & 0.601 & 0.520 & 0.000 & 0.140 & 3.325 \\
\hline Dichloromethane & 0.387 & 0.570 & 0.100 & 0.050 & 2.019 \\
\hline Methanol & 0.278 & 0.440 & 0.430 & 0.470 & 0.970 \\
\hline Acetonitrile & 0.237 & 0.900 & 0.070 & 0.320 & 1.739 \\
\hline Water & 0.000 & 0.450 & 0.820 & 0.350 & 0.260
\end{tabular}

Table 4.3.0: Solvation parameters of different solvents used in all the experiments. ${ }^{1} \boldsymbol{R}^{2}$ is the excess molar refraction that can be calculated form refractive index or can rather be easily estimated and ranges from -

0.240 (Isoflurane) $\rightarrow 2.29$ (Anthracene). $\mathbf{p} 2 \mathrm{H}$ is the solute dipolarity-polarizability obtained to date from gas-liquid chromatography of solutes on polar stationary phases and ranges from -0.20(Sulfur hexafluoride) $\rightarrow$ 1.72(4-Nitrophenol). a2H is the solute hydrogen bond acidity and ranges from

0.00 (Cyclopentane) $\rightarrow 0.95$ (Trichloroacetic acid). $\mathbf{b} 2 \boldsymbol{H}$ is the solute hydrogen bond basicity and ranges from 0.00(Cyclopentane) $\rightarrow 1.21$ (Tri-n-butyl phosphate). lastly $\log \boldsymbol{L}$ is the solute-gas-hexadecane partition coefficient at $298 \mathrm{~K}$ and ranges from -0.120(Sulfur hexafluoride) $\rightarrow$ 7.632(Phenanthrene)

4.4 - Dynamic Equilibrated Responses

\subsection{1 - Constant Total Flow Rate $(400 \rightarrow 420 \mathrm{sccm})$}

Subtle changes in vapor concentration mimic the gradual changes that we experience moving towards or away from an odor source to replicate the slow changes that are perceived in the background. ${ }^{86}$ (Figure 4.4.1a) The intensity factor follows the concentration changes over time. (Figure 4.4.1b) When PCA is applied to the $\Delta \mathrm{R} / \mathrm{R}$ matrix (using the same weights determined for the equilibrated $5 \% \mathrm{P} / \mathrm{P}^{0}$ $\Delta \mathrm{R} / \mathrm{R}$ data) the response extends linearly outwards and returns back to the $(0,0)$ origin. (Figure 4.4.1c) R $\mathrm{R}^{2}$ values for the principal components are 0.974 (Figure 4.4.1c) compared to an $\mathrm{R}^{2}$ of 0.895 for the equilibrated temporal responses (Figure 4.2.2). Using the intensity factor to separate out the concentration variable, the 
result is a stable concentration independent response. (Figure 4.4.1d) By this technique, the seemingly low and less linear portion of the intensity factor is responding linearly with concentration (Figure 4.4.1b) and this corresponds well with the majority of the scattered points within the concentration independent principal components (Figure 4.4.1d). As stated earlier, the sensors within this work do not typically respond well below concentrations of $2.5 \% \mathrm{P} / \mathrm{P}^{0}$ for methanol and the quantitative response cannot be deemed reliable. Compared to other forms of correction, this method is simple and based on the input intensity of the sensor array at each individual measurement. It does not overcorrect the gradual responses for concentration drift over time. ${ }^{21}$ 

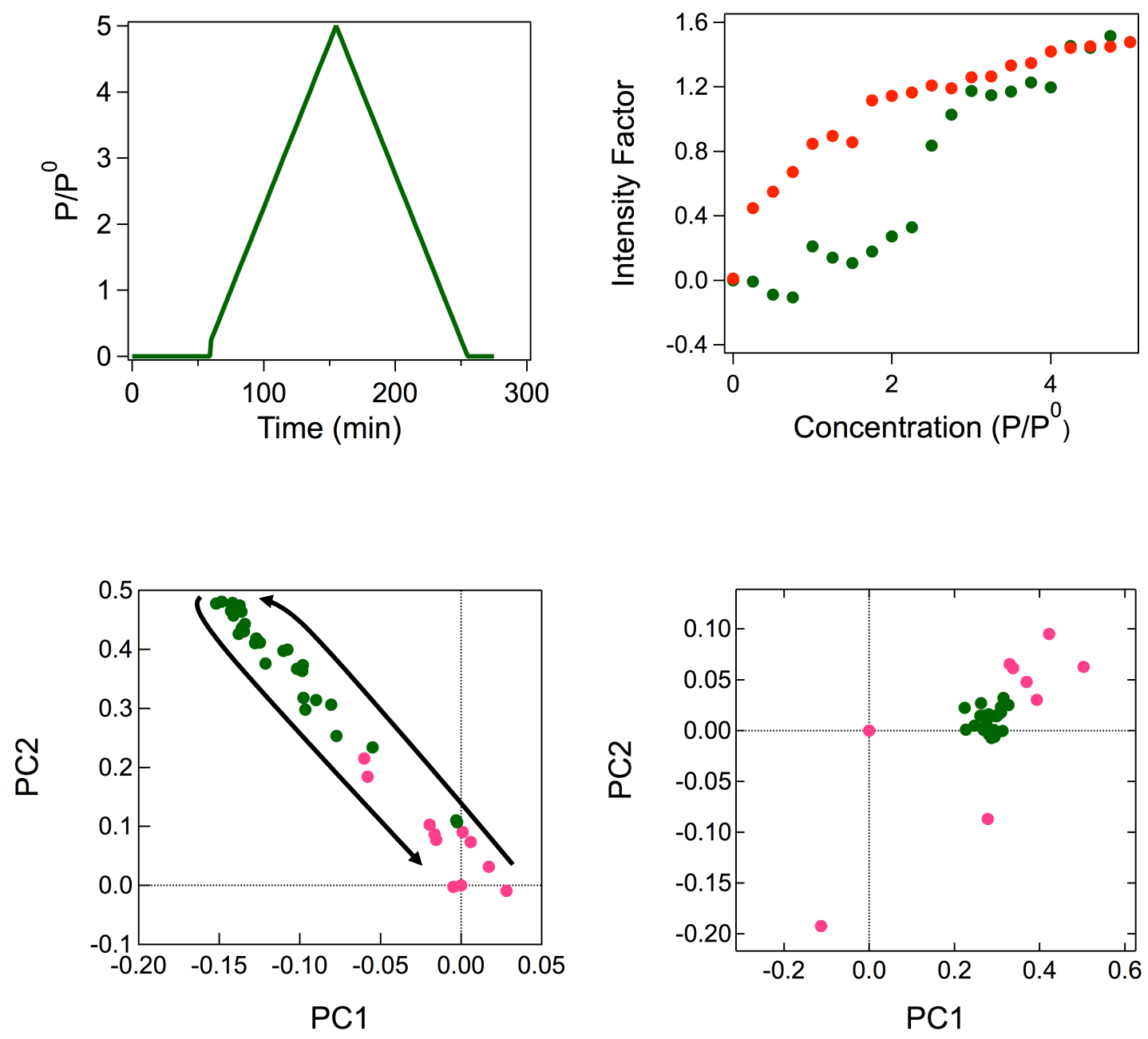

Figure 4.4.1: a)[top left] Partial pressure applied over time to the sensor array. The concentration was gradually raised to a maximum of $5 \% \mathrm{P} / \mathrm{P}^{0}$ at a rate of $0.25 \% \mathrm{P} / \mathrm{P}^{0}$ every $5 \mathrm{~min}$. $\boldsymbol{b}$ ) [top right] Intensity factor changes with concentration. Rise (green) $R^{2}=0.921$, fall (red) $R^{2}=0.847$. c) [bottom left] PC1 and PC2 response of $\triangle R / R$. d) [bottom right] Response of PC1 and PC2 for normalized data using the Intensity factor. Noisy, randomly scattered data points below the threshold intensity are marked in pink. Significant variance ensues below $2.5 \% \mathrm{P} / \mathrm{P}^{0}$ (3.95 ppt) at $25 \stackrel{\mathrm{C}}{\text { and }} 1 \mathrm{~atm}$. Points at $(-0.049,1.20)$ and $(6.56,2.73)$ are randomly scattered within principal component space and have been omitted from the image for a better visual of the cluster.

Gradual ramping up and down of the concentration changes the resistance up to a maximum of $5 \% \mathrm{P} / \mathrm{P}^{0}$. (Figure 4.4.1a) The gradual change in concentration can also be seen as a replication of moving towards and away from an odor source. 
The intensity factor follows the concentration, increasing up to a maximum at $5 \%$ $\mathrm{P} / \mathrm{P}^{0}$ and returning back close to the origin $(0,0)$ at $0 \% \mathrm{P} / \mathrm{P}^{0}$ (Figure 4.4.1b). PCA of the $\Delta R / R$ data is then performed on the $\Delta R / R$ matrix using the weights determined from the maximum $\Delta \mathrm{R} / \mathrm{R}$ of each vapor at $5 \% \mathrm{P} / \mathrm{P}^{0}$ (Figure 4.4.1c). Normalization using the intensity factor then PCA shows the changing response, below a given concentration. Even at equilibrated values, the points are scattered within principal component space. At approximately $2.0 \% \mathrm{P} / \mathrm{P}^{0}$ the points in the principal component space begin to cluster within the normalized PCA. (Figure 4.4.1d)

\subsection{2 - Constant Total Flow Rate (400 sccm)}

Comparison of a varying flow rate (Figure 4.4.1), where the vapor might be caught within a breeze, compared to a more static flow rate test (Figure 4.4.2), where the vapor is present within the current flow demonstrated characterization under different dynamic conditions. With increasing flow rates the vapor does not get caught up on the first few sensors as much, which initially depletes it from the vapor trail. Once the sensor's absorption rate decreases as it becomes more saturated, the responses of the other sensors then also increase, as more analyte is available downstream. ${ }^{93}$ Similar effects are observed within olfactory sense of human when sniffing. ${ }^{94-96}$ This experiment differs in that it is slowly changing only the odor rather than a slight increase with flow rate to simulate a "sniff". The implications of this test represent the effects of flow rate on the perceived odor. The effect of increasing flow rate is that it increases the rate of equilibration. ${ }^{97}$ 

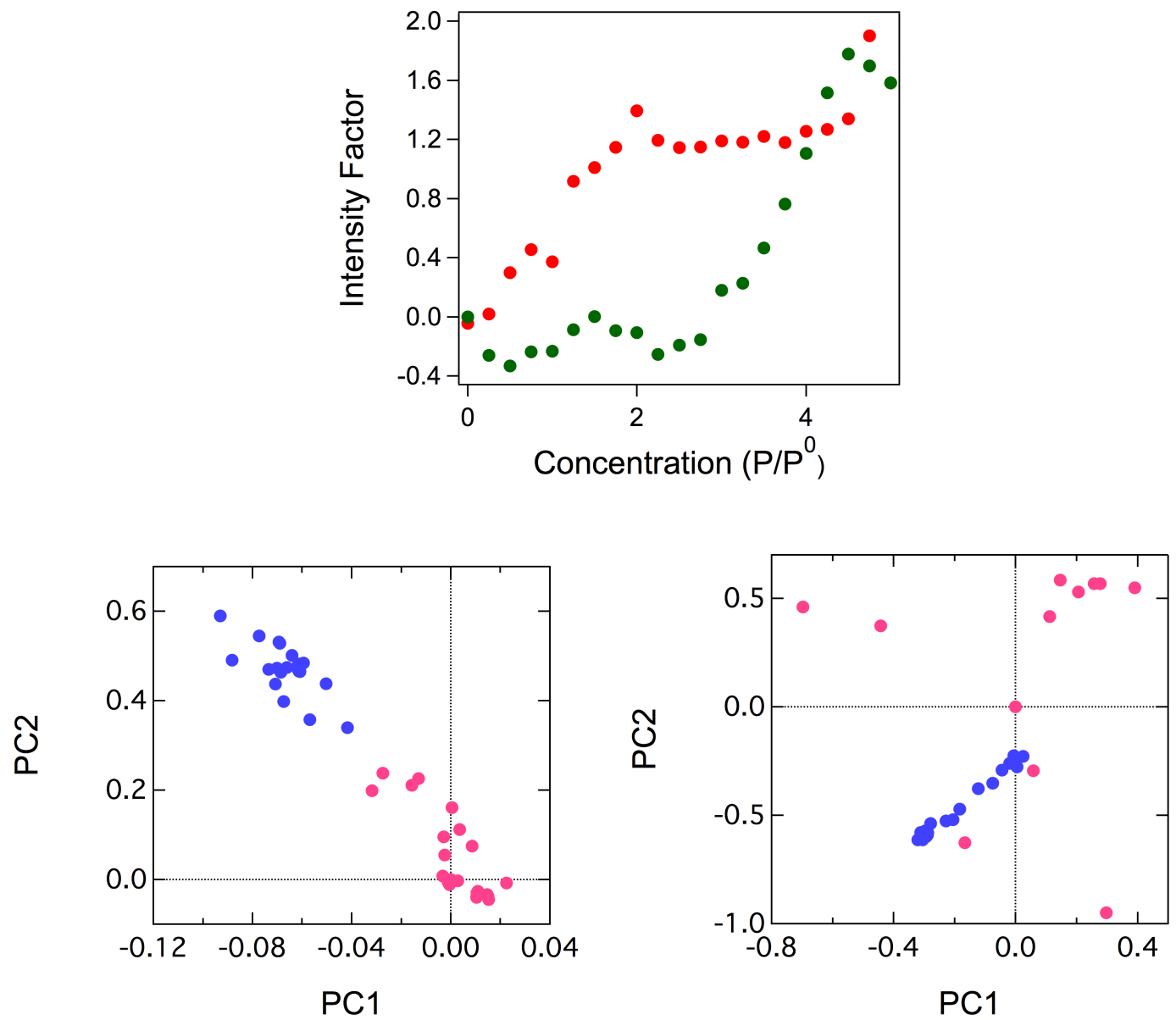

Figure 4.4.2: a) [top] Intensity factor changes with concentration. Rise (blue) $R^{2}=0.0 .738$, fall (red) $R^{2}=0.759$. b) [bottom left] $P C 1$ and $P C 2$ response of $\triangle R / R$. c) [bottom right] Response of PC1 and PC2 for normalized data using the Intensity factor. Points at $1.5 \% \mathrm{P} / \mathrm{P}^{0}(-13.173,30.878)$ on the rise as well as $0.25 \% \mathrm{P} / \mathrm{P}^{0}(-0.15539,5.2457)$ and $0.0 \% \mathrm{P} / \mathrm{P}^{0}(0.057149,-1.2535)$ on the return have been omitted from the figure as they are randomly scattered (pink points). (48 sensor array)

\subsection{3 - Time Delay in Response}

The calculated theoretical resistance at different time constants is plotted against the concentration as a measure of ensuring that the rate of concentration increase is allowing the sensor arrays to equilibrate.(Figure 4.4.3) The calculations were done using different saturation times based on the sensor arrays time constants. The longest time to reach one time constant is 9.78 minutes for PMMA 
exposed to toluene. The longest time constant including standard deviation is 11.92 for PMMA exposed to DCM. (Table 4.1.1) Comparisons of the calculated resistance to the concentration rise and fall maintain the same profile over time. There is no significant difference between the time constants used of 1,6 , or 12 minutes for this rate of increasing the partial pressure.

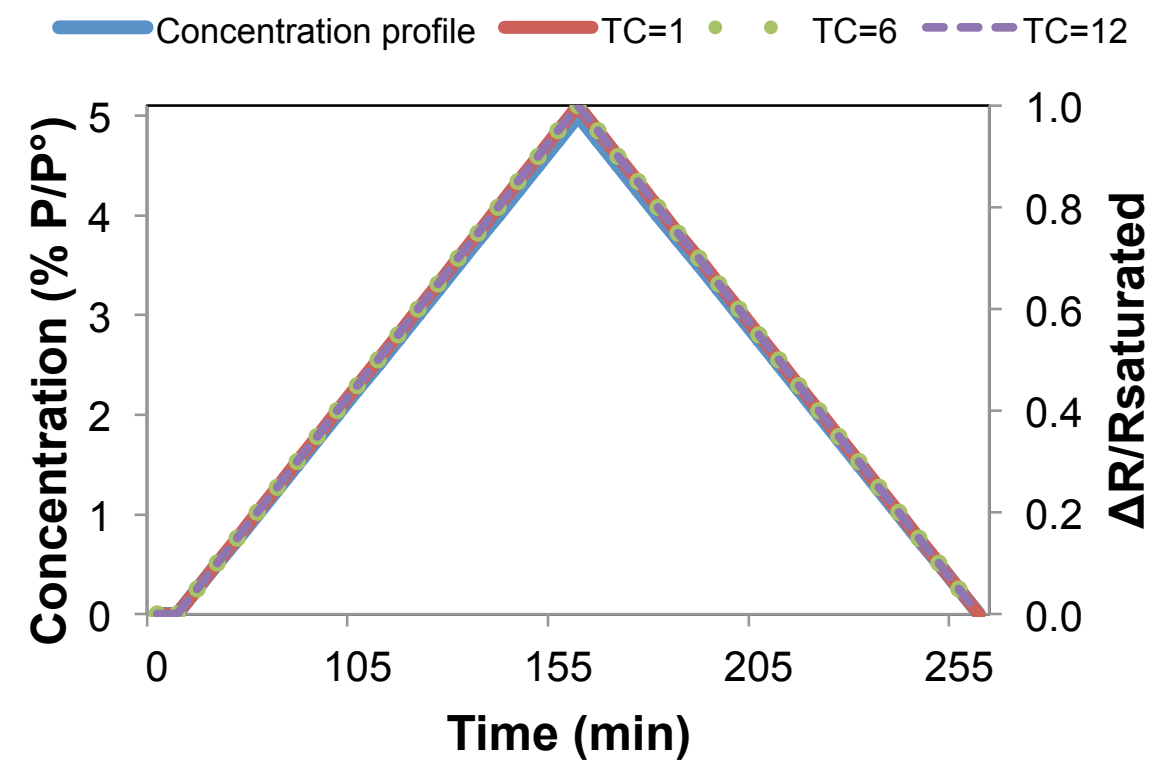

Figure 4.4.3: Theoretical modeling using the time constant with the slow changing flow rate used in the gradually changing partial pressure of methanol. The concentration profile (blue) is plotted over time. Time constants 1 minute (red), 6 minutes (green) and 12 minutes (purple) are used to calculate the $\Delta R / \Delta R_{\text {saturated }}$ over time.

\section{5 - Multiple odors}

Exposure to multiple vapors simultaneously adds complexity to the temporal identification of analytes. This is done here by building on the slow exposure of one analyte with a quick exposure of a second analyte (Figure 4.5.1). The sensor array is initially exposed to toluene using the same weights as the equilibrate sensor 
response at $5 \% \mathrm{P} / \mathrm{P}^{0}$ so all vapors should place the equilibrated toluene response within the same position in principal component space. (Figure 3.2.1) The addition of a second vapor on top of the first is predicted to move the principal components to a fractional distribution between the two (methanol and toluene) roughly where acetonitrile is positioned at its equilibrated value. ${ }^{24}$

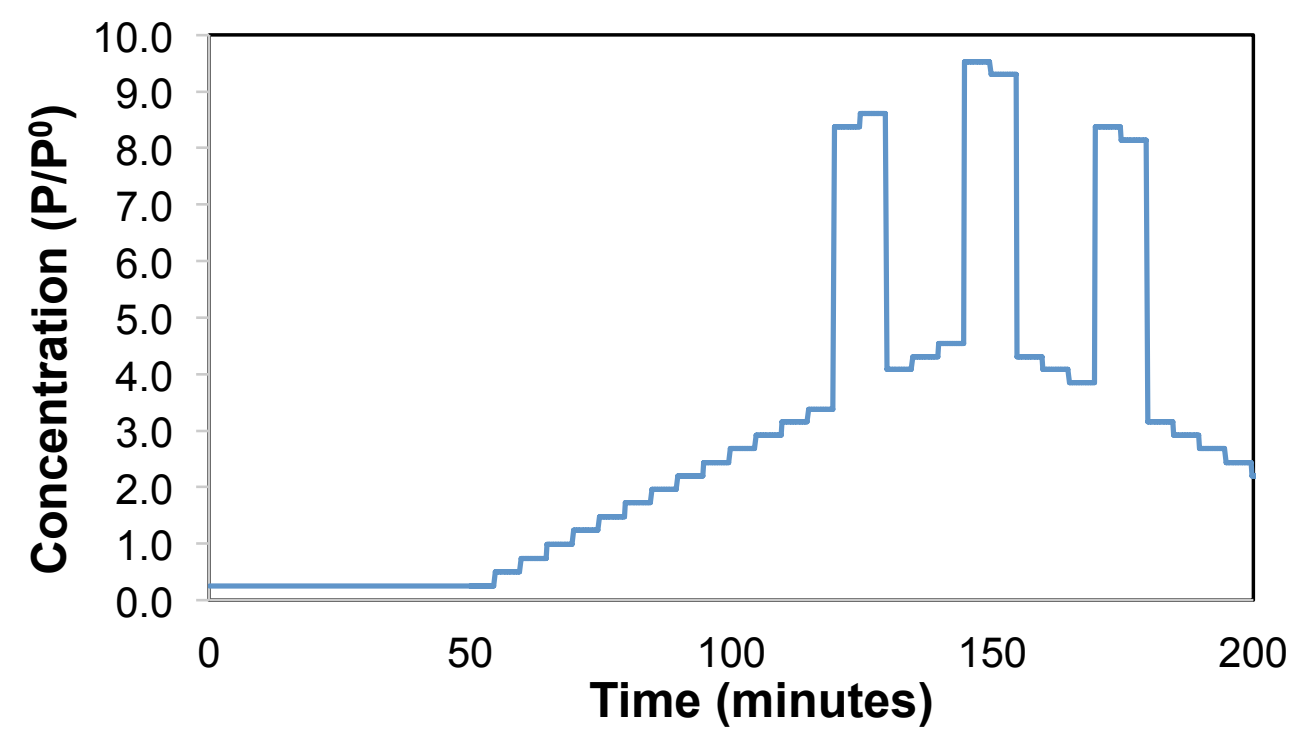

Figure 4.5.1: Concentration profile of gradual exposures of toluene up to a concentration of $5 \% \mathrm{P} / \mathrm{P}^{0}$ with a fast changing exposure of methanol at $5 \%$ $P / P^{0}$.

An analysis of the intensity factor demonstrated that the sensor array responds quickly to toluene once it passes the detection threshold of the sensor array becoming fairly linear, as it is able to keep up with the slowly changing background. (as demonstrated in Figure 4.5.2) Three peaks of methanol can be seen as the sensor array is exposed to $5.0 \% \mathrm{P} / \mathrm{P}^{0}$ methanol. The methanol peaks do not appear to reach saturation, but it is difficult to be sure with the changing toluene 
background adding to the peak height are making the peaks look more pointed rather than flat near the equilibration point.

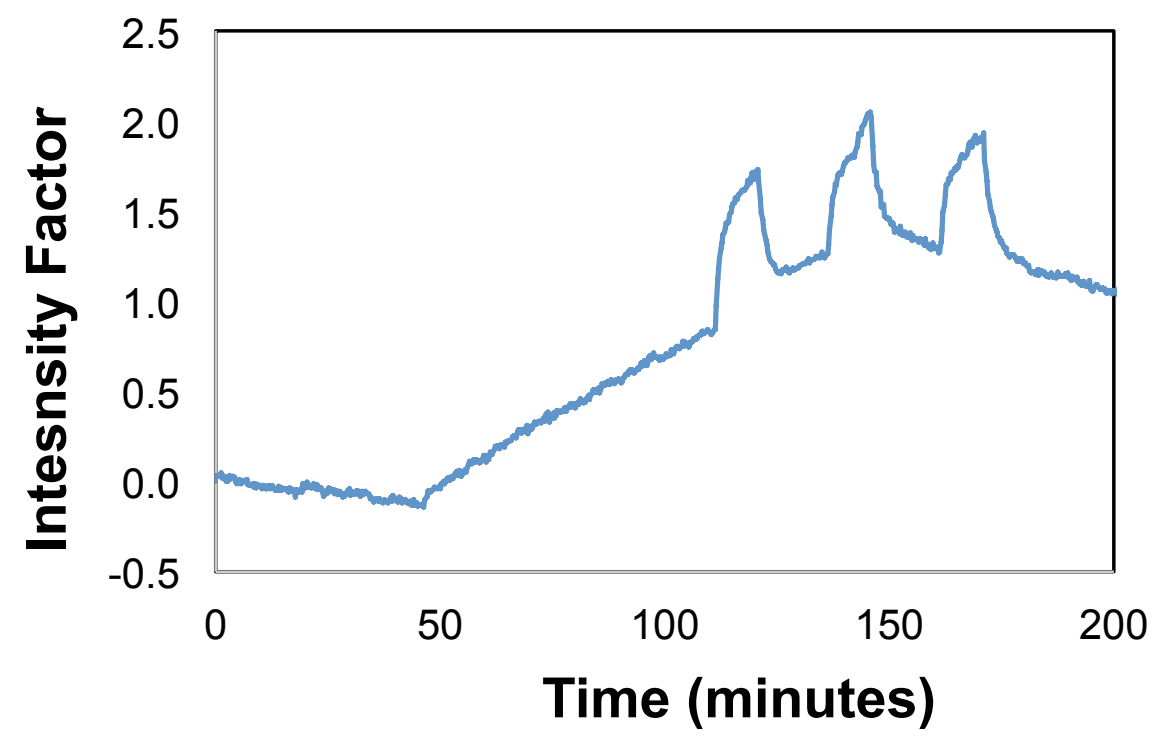

Figure 4.5.2: Temporal response of intensity factor when exposed to a slow background of toluene up to $5 \% \mathrm{P} / \mathrm{P}^{0}$ and a quick changing vapor.

Using PCA on the $\Delta \mathrm{R} / \mathrm{R}$ matrix the principal components they are initially spread out in principal component space as expected for the equilibrated toluene exposure at $5 \% \mathrm{P} / \mathrm{P}^{0}$ (as seen in Figure 3.2.1). A mixture of the two vapors does produces a fractional mixture of the vapors principal components. ${ }^{26}$ (Figure 4.5.3) The fractional mixtures within the principal component space form with a roughly equal proportion of the two analytes. To compare responses within principal component space half of the methanol response from Figure 3.2.1 are -0.09, 0.25, 0.01 , while for toluene it is in $0.06,0.02,0.02$ in PC1, PC2, and PC3 respectively. The equilibrated response within the principal component space of the mixture of 
vapors is $0.08,0.17$ and 0.04 in PC1, PC2, and PC3. Half of the combined methanol + toluene responses give $-0.03,0.32$ and 0.02 in PC1, PC2 and PC3 which are fairly close to the actual movement within the principal component space. ${ }^{26}$

The results show a potential that there is a misclassification by PCA because location of the points within PCA when using the same weights appear where acetonitrile is $0.038,0.27$ and -0.10 in PC1, PC2 and PC3 respectively. (Figure 3.2.1) The largest separation of the points is within the 3rd principal component to distinguish them, but in a more complicated mixture this becomes an even more complex problem. Incorporating time constants into the analysis as an added distinguishing feature could separate more complex vapor mixtures.

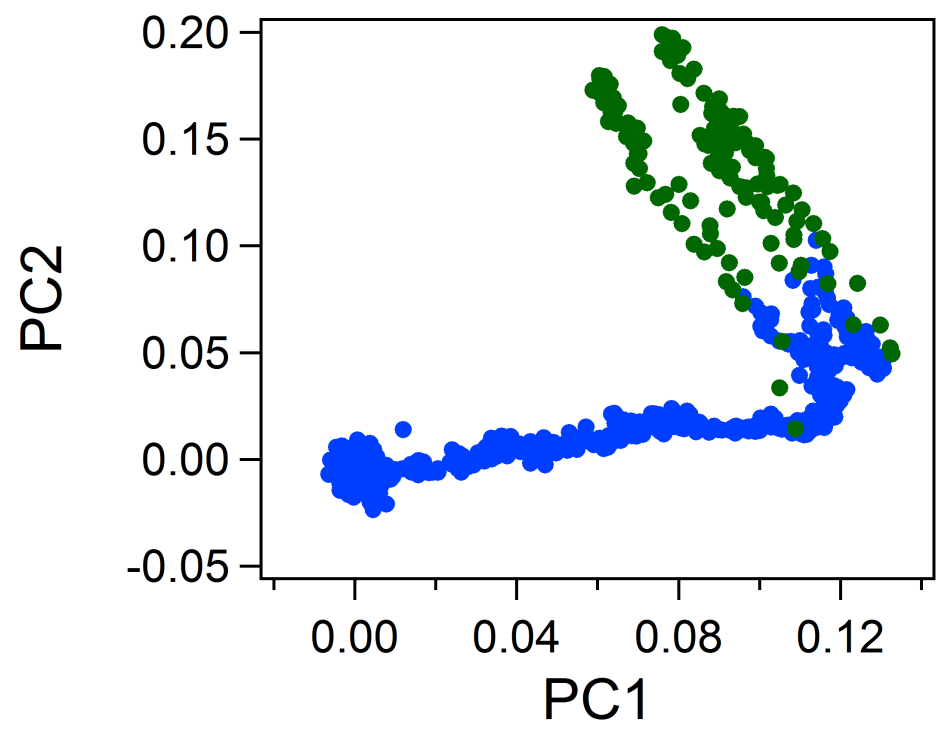

Figure 4.5.3: Principal components of sensor array exposed to a slow background of toluene (blue) up to $5 \% \mathrm{P} / \mathrm{P}^{0}$ and a quick changing vapor of methanol (green) at the same time. 
Using the intensity factor to separate the concentration dependent component, the normalized data appear to cluster tightly near the origin. The methanol peak forms a tight cluster at the center of the toluene peak. (Figure 4.5.4a) These data demonstrate the need for some form of filtering or removing additional points within PCA for a clearer picture to apply this method of analysis to real-time data. As the quantity of data becomes scaled up, a better method for cleaning up the data is needed so that the system and the user are not overwhelmed with information on the sensor array response.

Enhancing the image over the cluster shows the temporal response within the principal component space. (Figure 4.5.4b) The toluene cluster is clearly separated from the methanol cluster, except for the gradual transition to and from the methanol cluster. Comparison of these components within principal component space methanol is $0.211,-0.027,0.010$ PC2 and for toluene is $-0.084,-0.089$ and 0.011 for PC1, PC2 and PC3 respectively. (Figure4.2.3) The combined methanol + toluene exposure is $0.001,-0.067$ and 0.001 in PC1, PC2 and PC3 respectively. (Figure 4.5.4b) This is also close to the expected values of a combination of the two being $0.065,-0.058$ and 0.010 in PC1, PC2 and PC3 respectively. ${ }^{26} \mathrm{~A}$ better separation of the components is observed within the normalized PCA space as the analytes are clustered. Removal of slow changing data points should help the analysis by better defining the clusters and potentially removing the toluene peak entirely. 

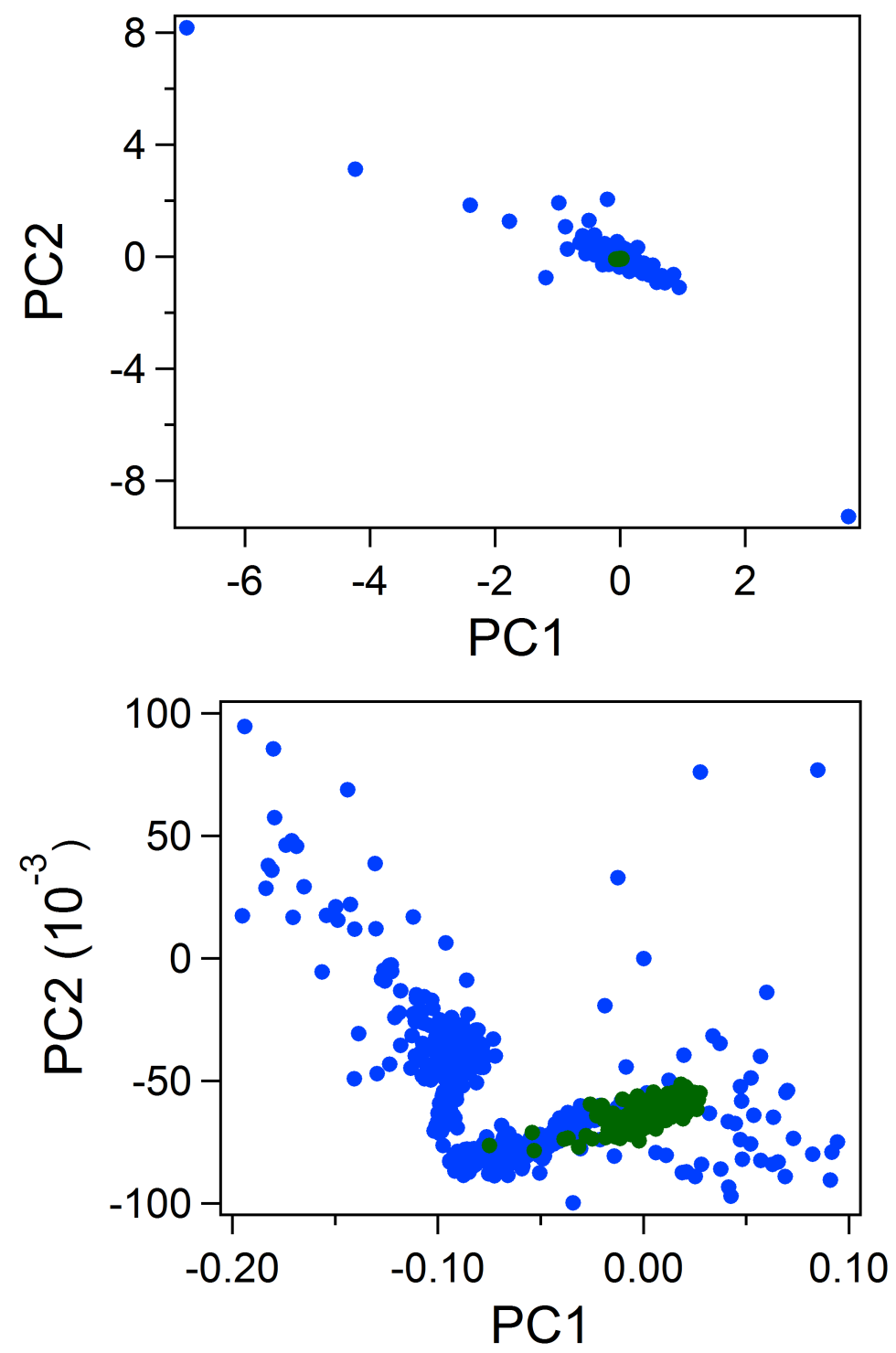

Figure 4.5.4: a) [top] Concentration independent component of gradual exposures of toluene (blue) up to a concentration of $5 \% \mathrm{P} / \mathrm{P}^{0}$ with a fast changing exposure of methanol (green) at $5 \% \mathrm{P} / \mathrm{P}^{0}$. b) [bottom] zoomed in profile of the normalized concentration independent pattern of $\boldsymbol{a}$.

\section{6-Filtering}

Filtering the data is a method of drift compensation for the variable background changes. (Figure 4.6.1) A filtering method was formulated for real-time analysis applications using two separate goals. The first is to reduce or eliminate the 
randomly fluctuating points within the principal components. (see Figure 4.4.1c) The second is to account for the baseline changes after exposure to a vapor. (see Figure 3.1.0 before toluene and after DCM)

Finding the maximum and minimum of the intensity factor sets provides the boundaries for the threshold response. For this experiment the threshold response was set to $1.0 \%$ of the intensity factor difference. For noisier data or less responsive sensors, the threshold can be increased to only identify intense responses if the background is noisier. Alternatively the threshold can be lowered to be more sensitive to smaller variations in sensor response.

The threshold filter works by monitoring the intensity factor and if it is below the set threshold response, then the $\Delta \mathrm{R} / \mathrm{R}$ values at that time are set to zero. If the intensity factor goes above the threshold, the $\Delta R / R$ remain the same (Figure 3.1.0). The filtered $\Delta R / R$ can then be used in PCA as well as for normalized PCA using the intensity factor. This method of filtration is similar to the way by which mammals have evolved to separate out background and only observe immediate changes within the environment. ${ }^{98}$ 

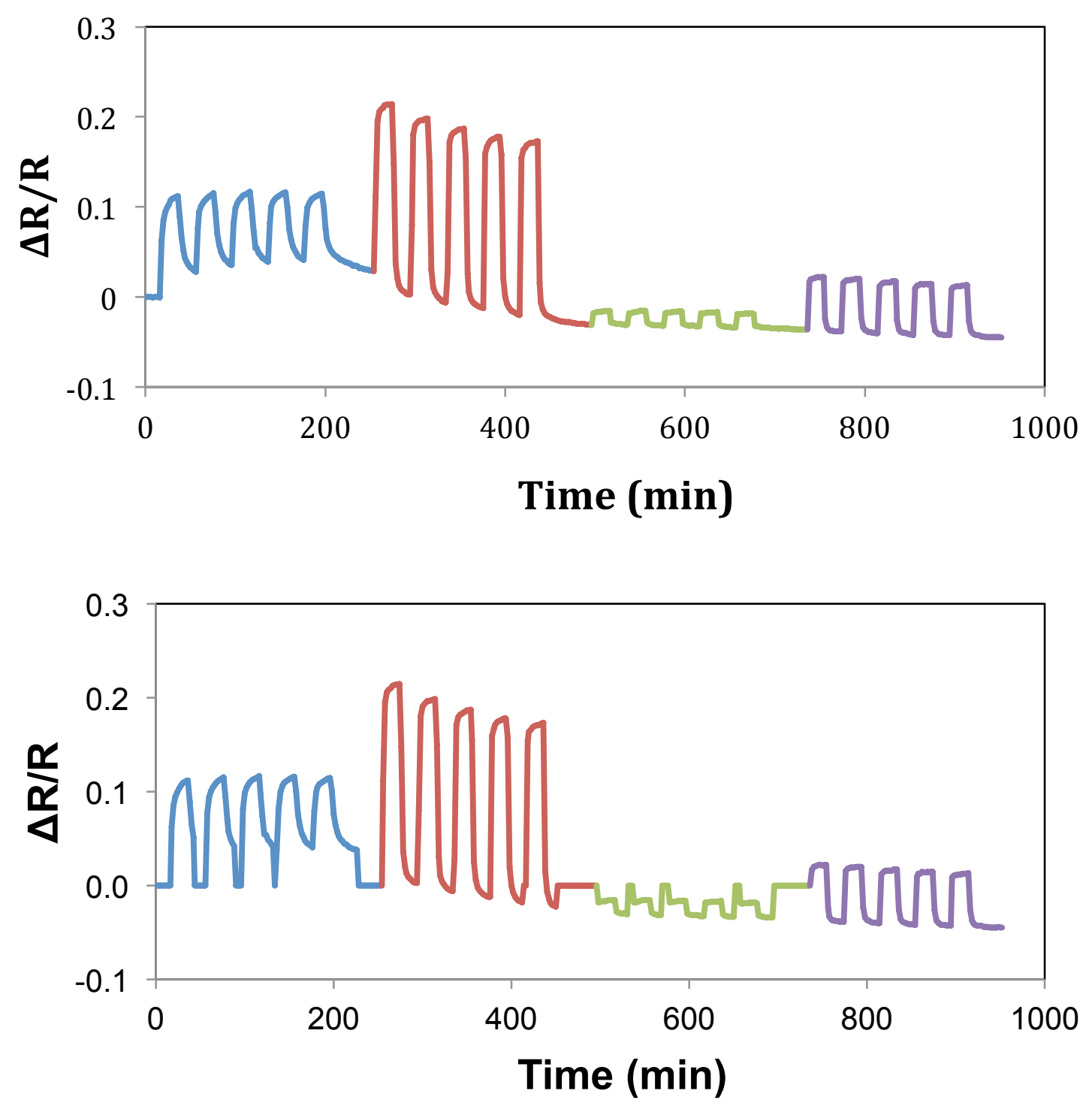

Figure 4.6.1: a) [top] unfiltered $\Delta R / R$ data are $5 \% \mathrm{P} / \mathrm{P}^{0}$ at a total flow rate of $400 \mathrm{sccm}$ for 4 vapors of toluene (blue), dichloromethane (red), methanol (green) and acetonitrile (purple). b) [bottom] Filtered $\Delta R / R$ data using the threshold set with the intensity factor. 
The advantage of this method is that it can be readily applied in the processing of data or for real-time analysis. (See section 5.2) However, upon applying PCA to the data, there are still large fluctuations within background that the filter will need to be accounting for to get a more accurate classification of the analytes. The points within the principal component space spread out from the origin $(0,0)$ and show a large separation between them once they have reached their equilibrated values. (Figure 4.6.2) Comparing these data to the unfiltered data (Figure 3.1.0) all the vapors are within similar points in principal component space. Classification between the analytes and the background in principal component space can slightly overlap as the vapor desorbs. Some sensors desorb certain vapors faster than others as demonstrated by the different time constants of the sensor array. 


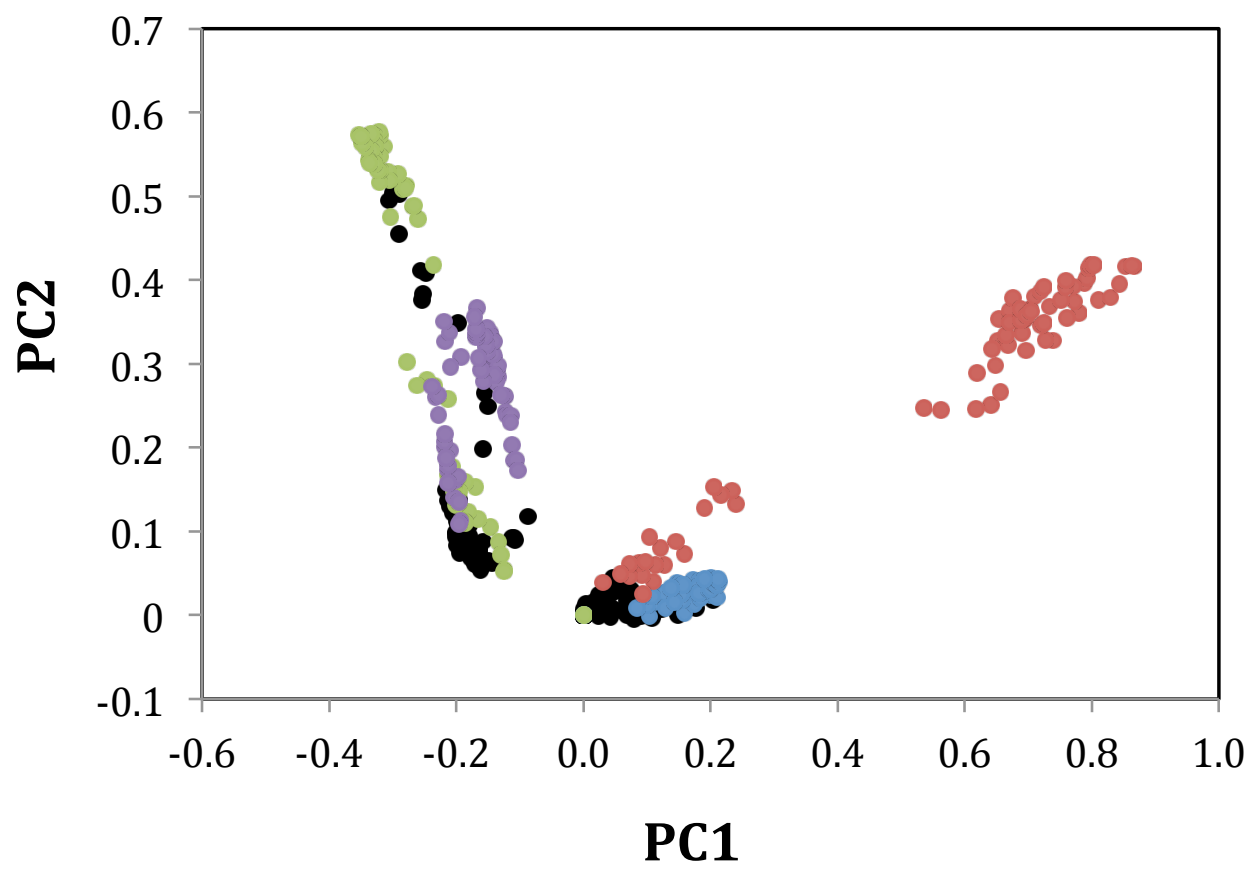

Figure 4.6.2: Filtered principal component data using the threshold set with the intensity factor. Data is $5 \% \mathrm{P} / \mathrm{P}^{0}$ at a total flow rate of $400 \mathrm{sccm}$ for 4 vapors of toluene (blue), dichloromethane (red), methanol (green), acetonitrile (purple) and black (background).

Normalization of the filtered data shows the separation between all of the vapors at their equilibrated values. (Figure 4.6.3) There is some crossover between clusters in the principal component space (Figure 4.6.2) between the unequilibrated $\Delta R / R$ of methanol and the acetonitrile cluster. The misclassification within the normalized $\triangle \mathrm{R} / \mathrm{R}$ PCA are caused by the filtering method being too rigid in its method of handling the changing baseline. By further making the baseline more adaptive the pattern can be identified in principal component space. This should reduce the contribution of the slow responding sensors creating a partial pattern. This should reduce the noise seen between clusters in PCA and potentially increase the separation between the analyte clusters. 


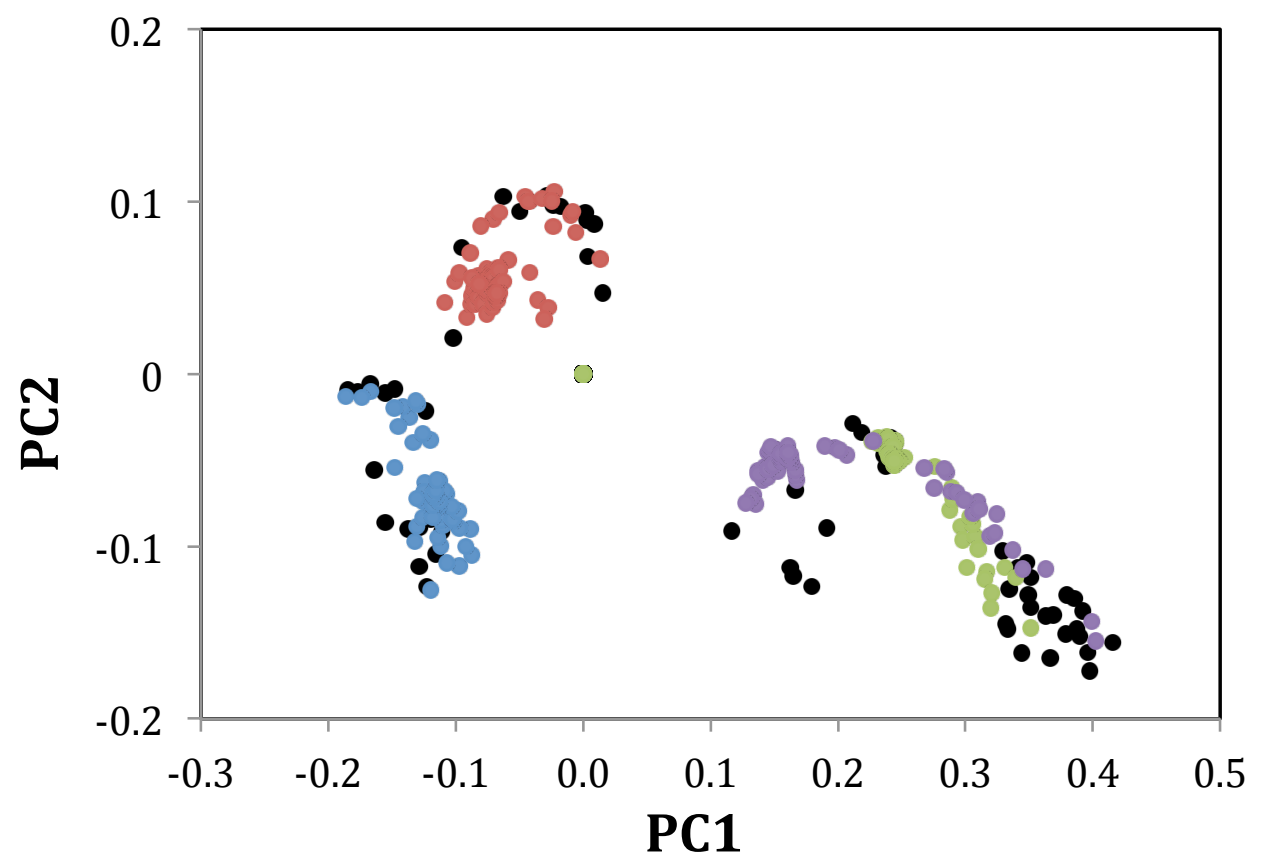

Figure 4.6.3: Filtered normalized principal component data using the threshold set with the intensity factor. Data is $5 \% \mathrm{P} / \mathrm{P}^{0}$ at a total flow rate of $400 \mathrm{sccm}$ for 4 vapors of toluene (blue), dichloromethane (red), methanol (green), acetonitrile (purple) and background (black).

\section{7 - Conclusions and Future Work}

Time constants are a unique characteristic to each sensor and vapor combination. Within an equilibrated sensor array, time constants are not necessary. When looking at dynamic environments, categorization and identification of similar points within the principal component space can be very challenging, due to drift. By developing a system that is capable of incorporating, understanding and learning the different time constants of each vapor, the time required for analysis, as well as the accuracy required will be greatly improved.

Slow gradual changes of an analyte to maintain equilibration of all sensors within the array do not exhibit the time-loops as discussed in section 4.2. The results demonstrate the effect of a concentration drift that could affect the sensor array and how 
PCA on its own is capable of identifying the odor because it does not form a consistent response. The normalized principal components are able to separate the concentration dependence and maintain a tight clustering of the analyte in the principal component space. A more complex scenario causes large crossovers between the analytes that are not always identifiable through PCA (Figure 4.5.4a). This is largely due to noise that is present from the background. A effective method of separating out these slow changes in the peaks is potentially found in filtering the data. Filtering is able to remove the small fluctuations within the background using the intensity factor. (Figure 4.6.1) However, the determination of the changing background is not sufficient and more flexibility needs to be added to the filter to adjust to a new background after an analyte peak.

Further testing of each sensor array with different vapors could also provide more information on the solvation parameters. Correlations and patterns determined from comparing solvation parameters of different sensor array responses to each analyte will give further insight into using the sensor array as a potential method of chemical analysis.

Future work for this chapter will be needed for testing of two or more analyte vapors that are slowly changing as well as fast changes in concentration. Additional analysis and development are also required for a filter that is able to adjust and separate the changing background from quick changes within the sensors. 


\section{Chapter 5: ANN \& Real-time analysis}

\section{$5.1-A N N \&$ Sparse Filtering}

An unsupervised ANN utilizing sparse filtering is analyzed and trained using data collected from a random exposure to 4 different vapors at $5 \% \mathrm{P} / \mathrm{P}^{0}$ (Figure 5.1.1). Additional factors for analysis within the ANN include the sparsity of the sample. The features are first divided by their norm, constraining them to fit within the $1_{2}$-ball (Figure 1.3.4). The sparsity factor then normalizes the features of the data for sparseness using a penalty that divides each feature by its mean square deviation.

The ANN is able to clearly separate out all 4 vapors from the background. There is also tight clustering with each designated vapor response, indicating associated spatial similarities for the data. ${ }^{14}$ The exception is the nitrogen gas background that has a larger degree of uncertainty, causing the larger spread around the cluster. The variance within the nitrogen background is most likely caused by the ANN deciding when and where the pattern has changed enough, so that it is no longer in the same class. This can be seen as faint black points between the nitrogen cluster and the other vapor clusters in Figure 5.1.1. Additional variation in the baseline resistance due to effects from the other vapors slightly shifting the baseline resistance are not fully understood but is a common problem causing sensor drift. ${ }^{59}$ Ideally the baseline resistance could be manipulated to normalize out the changes, keeping a consistent baseline. ${ }^{86}$ 


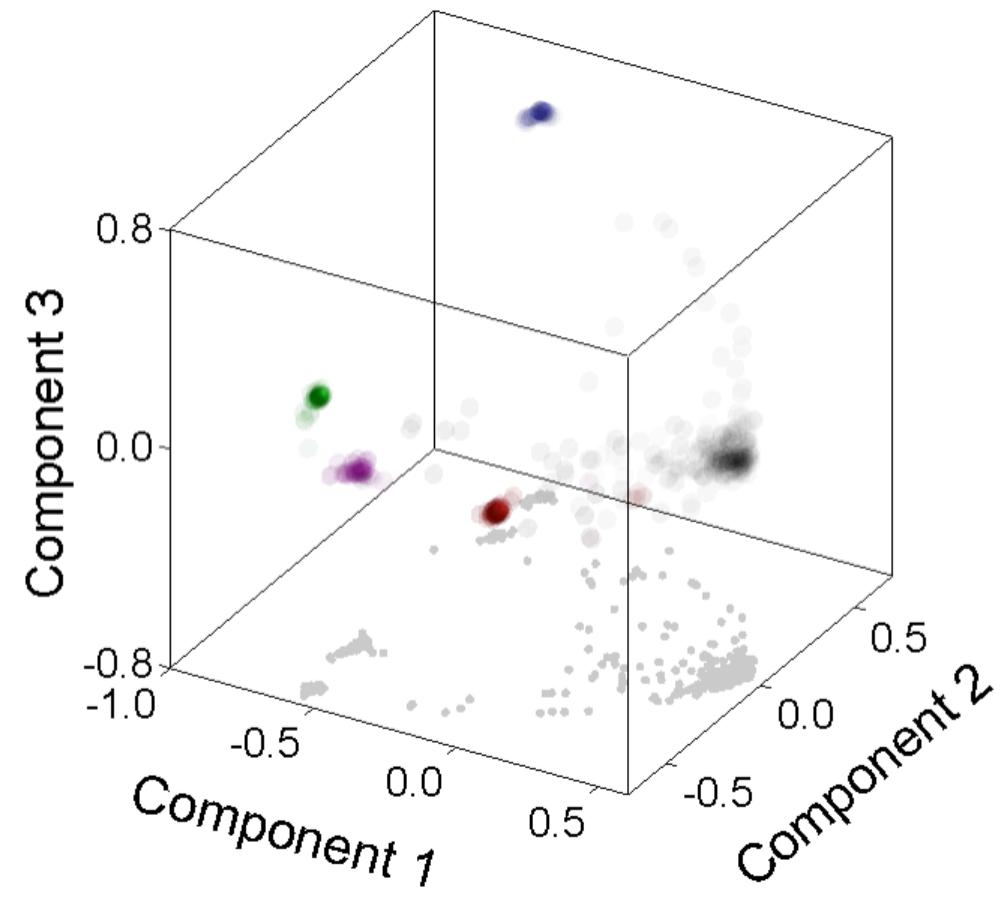

Figure 5.1.1: Sensor array was exposed to $5.0 P / P^{0}$ in 20 min on/off of toluene(blue), dichloromethane(red), methanol(green) acetonitrile(purple) and no analyte(grey) using ANN and a sparse autoencoder. Opacity represents the sum of responses where stronger measurements and increased point density are more visible.

Changes in concentration (Figure 3.3.0) add additional variance for each analyte cluster within the ANN.(Figure 5.1.2) A comparison of the repeated exposures at 5.0 \% $\mathrm{P} / \mathrm{P}^{0}$ (Figure 5.1.1) to the ramping of concentration for each vapor (Figure 5.1.2) shows less separation between analyte clusters, but a relative invariance vs. concentration changes. Analyte clusters are less defined and for some analytes there are multiple clusters. The largest difference is in the nitrogen clustering characteristics. Nitrogen clusters have decreased definition as well as 3 widely separated clusters. By looking at the temporal resistance data, there are different levels of nitrogen background through all the sensors that are perceived as a new odor to the unsupervised ANN. (Figure 3.3.0) 


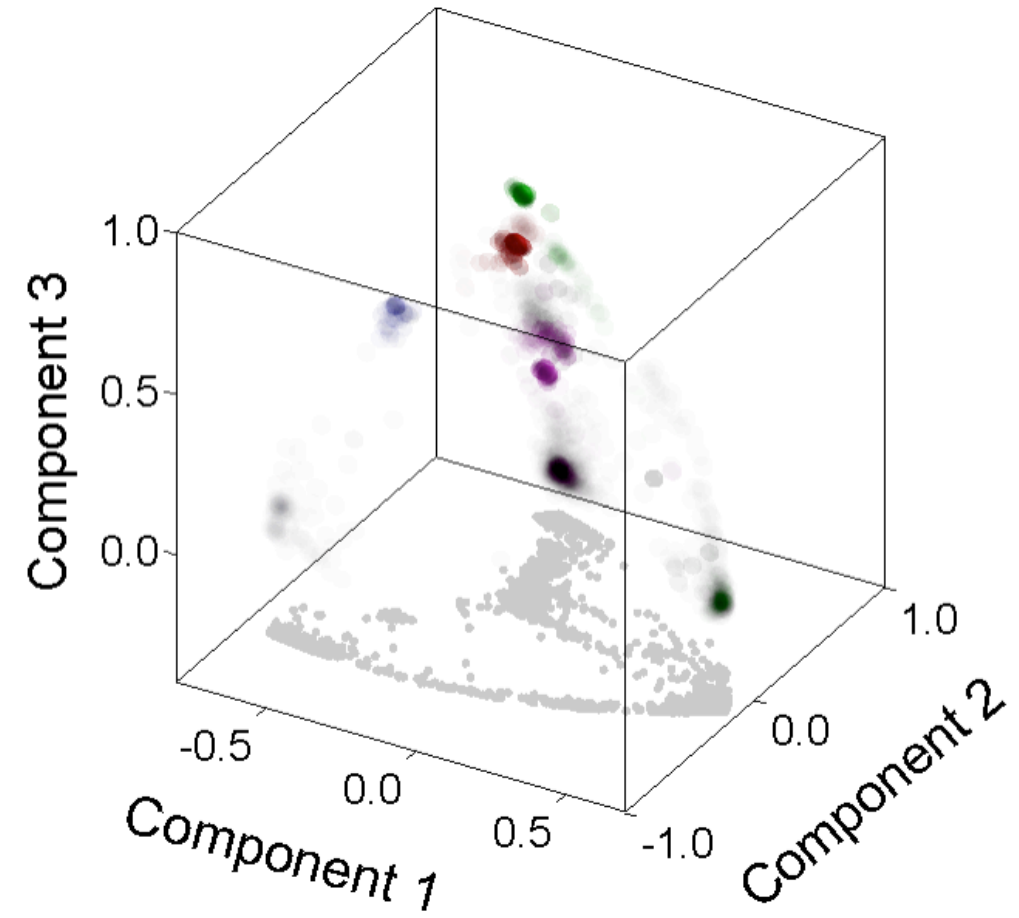

Figure 5.1.2: Sensor array exposed to toluene(blue), dichloromethane(red), methanol(green), acetonitrile(purple) and nitrogen(black) with increasing stepped saturated concentration responses of 0.5, 1.0, 1.5, 2.0, 2.5, 3.0, 5.0, 10.0 and 20.0\% $P / P^{0}$. A scatter plot is shown wherein the opacity points spotted is a sum of the responses elicited by the sensor.

With supervised ANNs separation is much larger and clearer (Figure 5.1.3) compared to the unsupervised method (Figure 5.1.1). There is tight clustering and very little random scattering. Acetonitrile tends to have a smaller response compared to the other analytes using this sensor array, this could be a portion of the cause for some of the increased variation number of points between acetonitrile and the background vapor.

(Figure 5.1.3) The artificial neural networks are capable of performing with greater amounts of data on a much higher level of analysis than using PCA alone, as shown by a quick comparison of Figure 4.2.2 (partial data set, using $\Delta R / R$ ) and Figure 5.1.1 (full data 
set). Further still to improve analysis is the use of the supervised method with sparse filtering (Figure 5.1.3), incorporation of multiple inputs (ex. machine vision) to speed up and guide the analysis will greatly improve the identification within chemical sensor arrays. A biologically comparable method of analysis is using our nose as well as our eyes to identify a potential odor. ${ }^{99}$

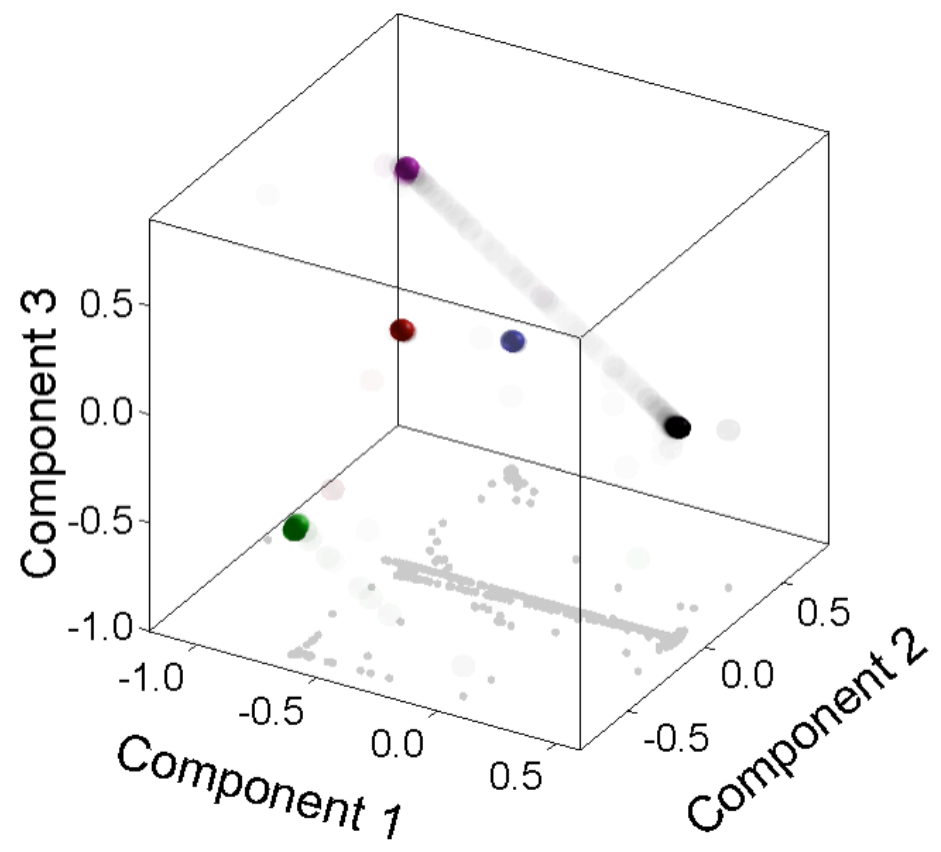

Figure 5.1.3: Sensor array was exposed to $5.0 \mathrm{P} / \mathrm{P}^{0}$ in 20 min on/off of toluene(blue), dichloromethane(red), methanol(green), acetonitrile(purple) and nitrogen(black) using ANN and with a supervised method. The weights were initially seeded using the sparse autoencoder. A scatter plot is shown wherein the opacity of points plotted is a function of the sum of responses elicited by the sensor. This has the impact of making stronger measurements more visible, and also provides a stronger sense of point density in the projected space.

\section{2 - Real-time PCA \& ANN}

A carbon black - organic polymer sensor array exposed to open atmosphere within the laboratory is able to maintain a stable baseline with minor fluctuations. 
Exposures to vapors were done at $25^{\circ} \mathrm{C} \pm 1$ and $101 \mathrm{kPa} \pm 1$. The sensor array used within this portion of the experiment consisted of seven sensors, one sensor of each type except for PMMA. The sensors were chosen for this sensor array because they are faster to most of the analytes (Table 4.1.1) and better behaved (Table 4.1.2) than PMMA. Using the filtering method described in section 4.6, the principal components of the filtered $\Delta \mathrm{R} / \mathrm{R}$ were then calculated. The analysis was done in real-time using Matlab as the data collection and processing program for the filtering and PCA. Data points were collected every 5 seconds over a period of one hour.

All sensors responded to the vapors with varying degrees of $\Delta \mathrm{R} / \mathrm{R}$. (Figure 5.2.1) Some sensors were noisy such as poly(n-vinyl pyrrolidone), possibly due to variations within the real-time, dynamic environment. Further analysis is required to determine the true cause of these variations. 


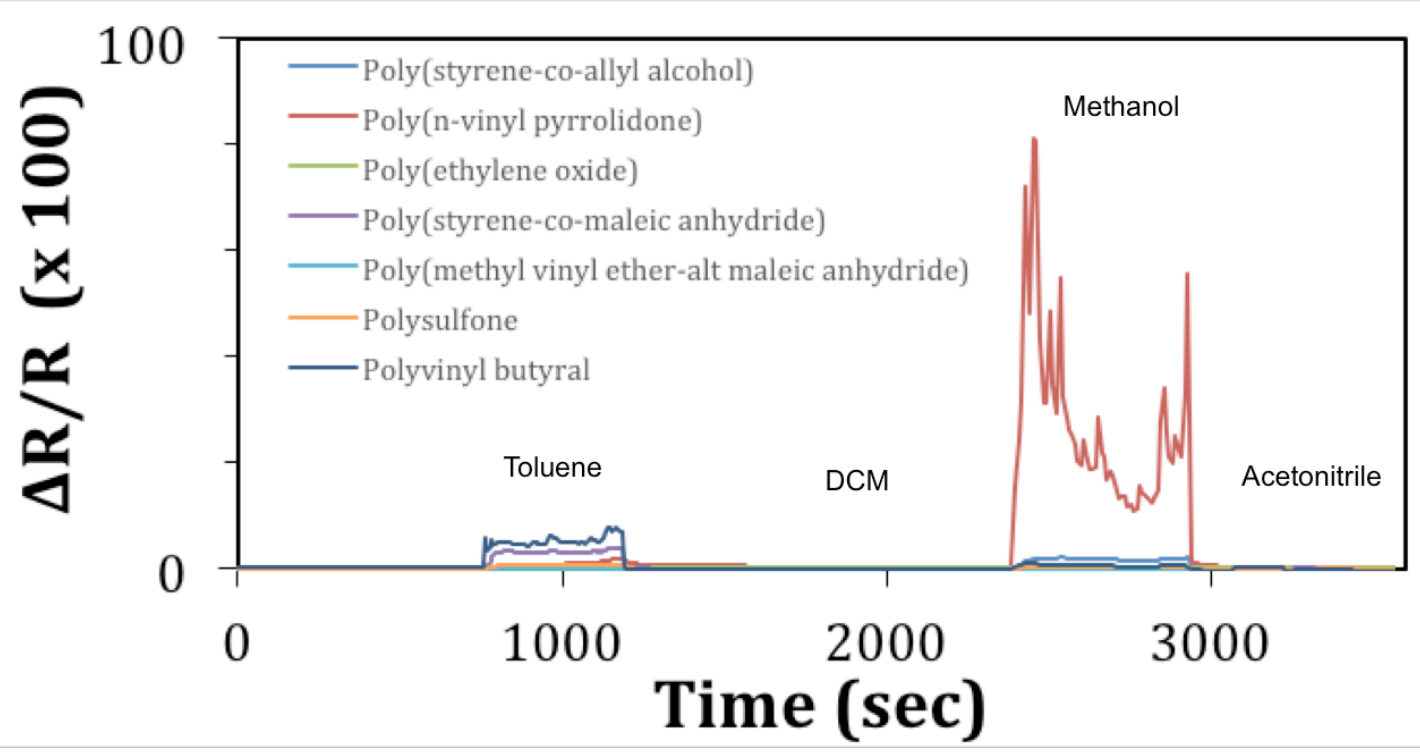

Figure 5.2.1: Filtered $\Delta R / R$ data for the real-time analysis of 4 vapors (toluene, dichlorormethane (DCM), methanol and acetonitrile) over one hour with a measurement every five seconds. A $1 \%$ threshold response (difference between the maximum and minimum of the intensity factor) was applied to the $\Delta R / R$ data.

Vapor clusters within the principal component space did not show large separation except for methanol from the other vapors, they instead formed wide clusters that partially overlapped with each other. (Figure 5.2.2a) This is likely because of the less controlled sampling method and this adds additional fluctuations within the sensor resistance response. This less controlled method of sampling means the analyte is not passed across the sensor in a steady flow. Instead the analyte vapor drifts across the sensor gently, with the possibility of a cross breeze disturbing the steady absorption into the sensor. The added variation in sampling methods as well as the sensor responses (dynamic background vapor) inherently add fluctuations in the vapor that need to be accounted for by using a filtering method. The filtering method used (see section 4.6) was not able to account for the level of dynamic variation that was seen based on the lack of 
separation. The background vapor is tightly clustered around $(0,0)$ because of the filtering method used. (Figure 5.2.2b) The characteristics of the methanol cluster gradually separated from the $(0,0)$ origin and settled in a cluster near $(0.5,6)$. The characteristics of dichloromethane are quite different, probably due to the large variations that were seen within poly(n-vinyl pyrrolidone). This caused the resulting principal component variation to spread out to $(-3.2,80)$, then return to form a cluster between 10-35 within the first principal component. Variations of this nature within sensor array analysis are not uncommon and may be due to the size of the sensor and the vapor interaction. ${ }^{46}$ Because sensors will exhibit more noise with large polymer/vapor partition coefficients that will strongly sorb the analyte and deplete the surrounding area. With uncontrolled vapor flow, the analyte is dependent on the environmental (breeze) and physical (pushing the air, convection) effects. ${ }^{46}$ 

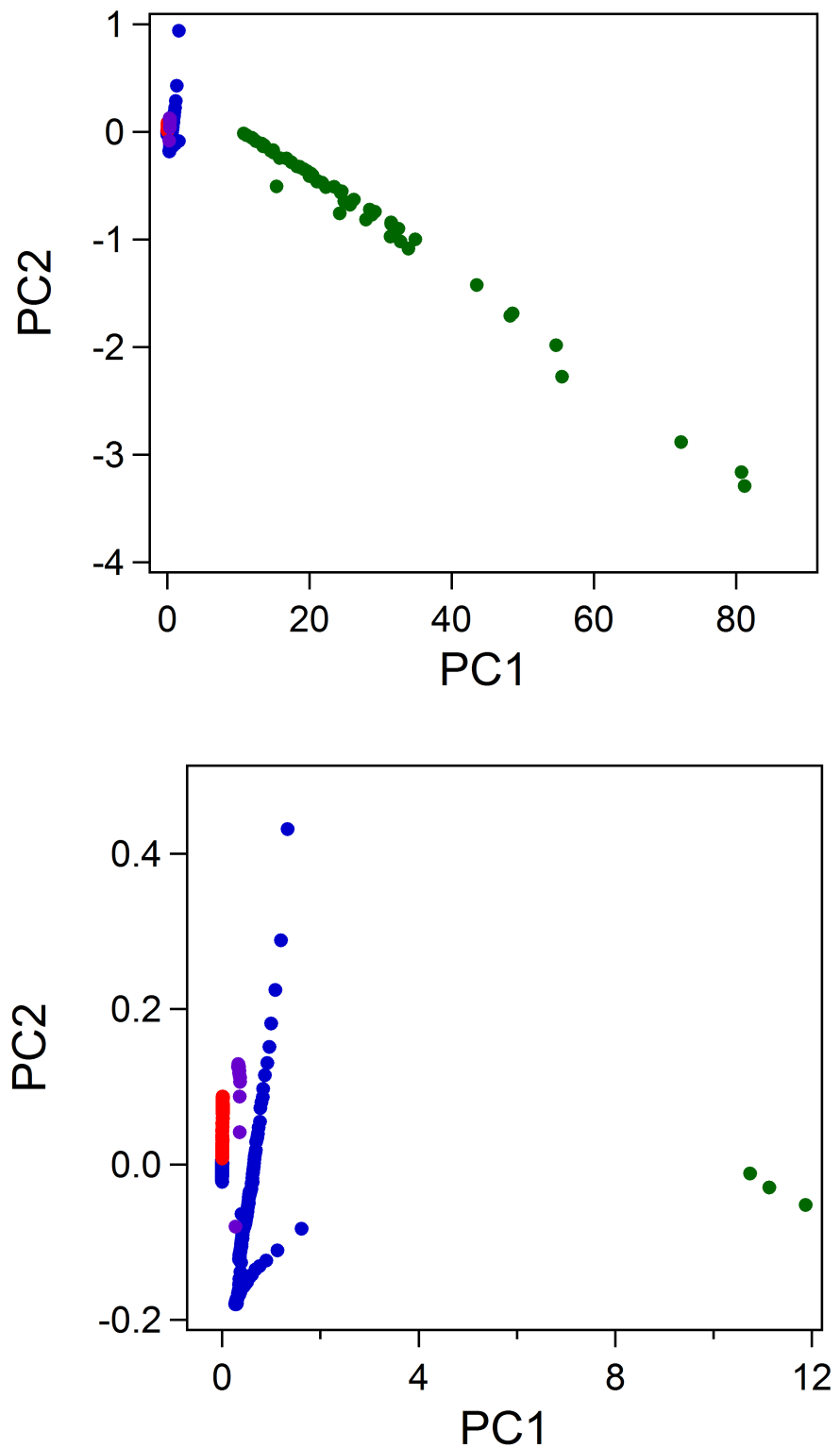

Figure 5.2.2: a) [top] PCA for the real-time analysis of four vapors within the open air of the laboratory for toluene (blue), dichloromethane (red), methanol (green), acetonitrile (purple) and nitrogen (black). Flow rate is uncontrolled as well as the concentrations of the analytes. b) [bottom] The enhanced image of the central cluster.

PCA of the concentration independent response pattern shows the clustering of the vapor analyte patterns in principal component space (Figure 5.2.3a). There is a large spread of the analytes along the diagonal axis through $(0,0)$ showing the low signal and 
large variation for those points. They are likely caused by noise within the sensor array and are just above the threshold value. Increasing the threshold value of the filter from $1.0 \%$ by a few percentage points should eliminate the additional noise.
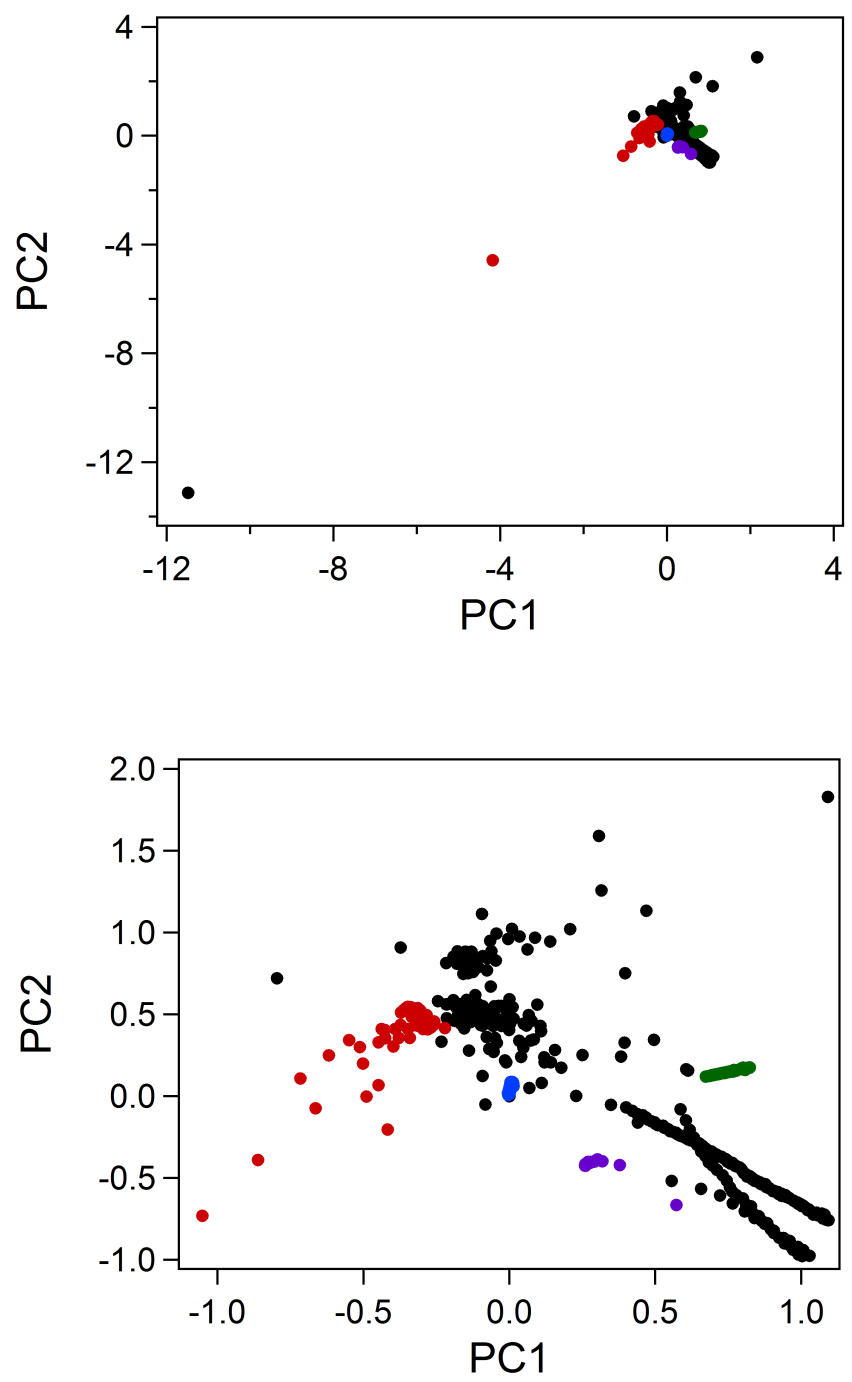

Figure 5.2.3: a) [top] $P C A$ using the normalized $\triangle R / R$ for the sensor array in real-time analysis. b) [bottom] The Enhanced image for viewing of central cluster of points in the PCA of the real-time analysis.

At the center of the grouping of all the clusters within principal component space the separation of individual anlaytes is masked by the drifting of the background ANN 
response. Surprisingly it is the dichloromethane that has the largest within cluster scatter of the four analytes and not methanol (as expected from the poly(n-vinyl pyrrolidone response (Figure 5.2.1) as well as the principal component scatter (Figure 5.2.3b)). A potential artifact within the real-time analysis (Figure 5.2.3b) is the drifting baseline which results from the different time constants of the sensors. The slow responding sensors take a while to desorb the analyte and the drifting response is observed (Figure 5.2.3b).

\section{3 - Real-time ANN analysis}

Analysis of the ANN was not achieved within this work due to a shortage of time. Future studies could apply the characterization methods learned and applied within this work into the ANN for real-time analysis. With ANNs operating in realtime there will be a larger influx of data that is streaming. The larger amounts of data would better train the ANN and improved performance in pattern recognition and classification would be expected.

\section{4 - Additional Considerations}

Considerations that can be included within a ANN for improved classification and function include using a method for improved filtering methods ${ }^{15,29}$, sensor selection ${ }^{27}$, sensor replacement and the addition of new sensors to the array. ${ }^{100,101}$ Improved filtering methods using ANN and sparse filtering will assist in the removal of unwanted noise and drift due to sensor degradation or variation from external factors (humidity, additional vapors). Selection of different sensors using ANN to determine which are responding 
first and the delay between responses can give additional information for analyte classification $^{27,102}$ as well as directional information for tracking an odor plume as demonstrated in mammals ${ }^{94,96}$ and some robots ${ }^{52,85}$. Additionally monitoring and identifying sensor behaviors within a ANN will aid in the long term use of the sensor array.

\section{$5.4-$ Conclusions}

ANN using sparse filtering are used to address and demonstrate the function of this architecture within the application of machine olfaction. Unsupervised methods with sparse filtering have shown a large improvement in the separation of vapor clusters (Figure 5.1.1) compared to PCA alone (Figure 4.2.2). Upon switching to the supervised method of analysis, the results further improved with a very low variance within the vapor clusters and far fewer points scattered randomly.

Unsupervised concentration ramping using the ANN and sparse filtering has shown that the added variance from concentration does not cause a drift outwards (Figure 3.2.1) but instead remains tightly clustered (Figure 5.1.2). The clustering is a large improvement to the normalized PCA alone (Figure 3.2.2) as it incorporates all of the raw data and not just the $\Delta R / R_{\text {saturated. }}$

Unsupervised methods demonstrated the effectiveness of this method even with a small sensor array (48 sensors) and using a small sample size ( $\sim 600$ data points). As ANNs obtain an increasing number of samples the average performance error should decrease, meaning that the ANN is getting closer to the desired output value. ${ }^{13}$ It would be expected that this ANN architecture's pattern analysis would only get better with more information presented to it. In addition the inclusion of such variables as additional 
filtering methods, sensor selection, sensor replacement and addition of new sensors to the array would increase the functionality and versatility of a sensor array incorporating such a ANN with sparse filtering.

ANN and sparse filtering was measured using real-time analysis. PCA results show that using the filtering method as well as the intensity factor as a method to separate out concentration is possible. Consideration of using multiple inputs for supervised methods of combined olfactory response mechanisms in ANNs. 


\section{Chapter 7: Conclusions and Future Work}

In the work presented within this thesis, temporal responses of chemically diverse arrays are a complex and information rich area that can be analyzed in real-time with application in ANNs. Temporal responses within sensor arrays are a large barrier in early identification of vapor analytes. This is especially true in dynamic environments where the sensor may never be at an equilibrated state and will dramatically slow and cripple the accuracy of the identification and quantification of the analyte.

Using the intensity factor to quantify the vapors the detection limits for each vapor were found to be to $1.7,19.7,7.9$, and 5.6 ppt at $5 \% \mathrm{P} / \mathrm{P}^{0}$. Using this method in more complex scenarios such as real-time analysis within a dynamic environment will improve the classification process because it removes the concentration variation from the $\Delta R / R$ of each sensor to give the concentration independent response pattern. This method works well but the changing shifts in the baseline resistance cause additional variance that can alter the $\Delta \mathrm{R} / \mathrm{R}$. A filtering method to remove such variations has been proposed but this method requires further work to fully reduce the large changes in background resistance. The design of the threshold function is flexible so that if the peak is positive or negative $\Delta \mathrm{R} / \mathrm{R}$ it will be able to filter out the response. Additionally by using the intensity factor the filter is flexible to any sensor because it uses the relative percentage of the sensors response. An additional feature that would enhance the filters function is using a more flexible variable that is able to change and reset the baseline when a new one is encountered.

Temporal analysis of the changing analytes has shown that there are unique time constants that can be attributed to each sensor and vapor combination. Incorporation of 
the time constants within the analysis will benefit the analysis in a minimum of two ways. First by reducing the time for analysis by monitoring the $\Delta \mathrm{R} / \mathrm{R}$ over time. Using the time constant equations and $\Delta \mathrm{R} / \mathrm{R}$ the time constant can be predicted as the analysis is proceeding. Secondly by knowing the time constant potentially the future behavior of the system can also be predicted. The purpose of using the time constants of vapors is to speed up the classification and identification of analytes as well as handling the complexity of multi-vapor dynamic system.

Testing of the sensor array using dynamic analysis demonstrates the impact of a slowly changing background on the principal component response. Using the intensity factor the concentration variance for classification is able to be controlled above the detection threshold. This is a similar exposure profile to the slow leak of a gas, a gentle breeze carrying the vapor, or slowly moving towards and away from a vapor source. Applying filtering methods to this type of exposure in the future will hopefully remove the slow changing responses of the background leaving the fast changing $\Delta R / R$ responses for identification. Variations in the flow rates will affect the responsiveness during the analysis. Faster flow rates are better for analysis because this increase the rate of equilibration of the sensors. The intensity factor is able to control the concentration variance in both the higher flow $420 \mathrm{sccm}$ and lower flow $400 \mathrm{sccm}$ systems maintain a tight clustering of the analyte response.

Moving to a mutli-vapor system with slow and fast variations in analyte partial pressures the sensor array is able to capture both analytes simultaneously. With the slow increasing response of toluene and methanol are captured by the sensor array and separated from the concentration independent component. Comparison of the principal 
components shows that the mixture (methanol + toluene) is close to the predicted fractional movement of the principal components for the cluster. Further analysis is needed to determine the effect of filtering and separation of the points when time constants are included within the analysis. It may be possible to use only the time constants alone to identify methanol from toluene in a two vapor system but further testing is needed.

Incorporation of the ANN and sparse filtering into the pattern analysis proved to be better at analyzing both the random exposure data (at $5 \% \mathrm{P} / \mathrm{P}^{0}$ ) and concentration ramping data. Using unsupervised techniques demonstrated that some of the baseline data is classified as a peak due the changes after an exposure. Application of a filter as described in section 4.6 and then ANN with sparse filtering will remove or at least reduce the number of false peaks. The use of a filter should also clean up any stray points that may appear within the ANN response as this is likely random noise within the sensor array. Supervised data had much less variance within vapor clusters (Figure 5.1.3) than the unsupervised method (Figure 5.1.1). Future work involving the use of a supervised method with another form of vapor identification such as machine vision may prove to be a very accurate efficient way of identifying vapors.

A more sophisticated analysis such as ANN with sparse filtering and incorporating the time constants may help for the identification of analytes. Seeing as the ANN with sparse filtering is already able to separate the analytes used in this work it would be of interest to use it in a ever increasing complexity of vapors. The addition of the time constant will add an extra degree of differentiation of the vapors because of the different polymer and analyte interactions. 
Transition of the ANN to real-time analysis was not completed due to the longer than anticipated timeframe for characterization and development of analysis methods for the sensor array. However the application of such should be fruitful as the ANN collects very large samples of data for analysis it can be trained on the streaming data. Use ANNs to incorporate additional features into the analysis (time constants, sparse filtering). The last step is to move the system to a real-time method using an ANN. 


\section{Works Cited}

(1) Abraham, M. H. Scales of Solute Hydrogen-Bonding: Their Construction and Application to PhysicochemicaI and BiochemicaI Processes. Chem. Soc. Rev. 1993, 22 (2), 73-83.

(2) Wilson, A. D.; Baietto, M. Applications and Advances in Electronic-Nose Technologies. Sensors 2009, 9 (7), 5099-5148.

(3) Wright, J.; Yang, A. Y.; Ganesh, A.; Sastry, S. S.; Ma, Y. Robust Face Recognition via Sparse Representation. IEEE Trans. Pattern Anal. Mach. Intell. 2009, 31 (2), 210-227.

(4) Chellappa, R.; Wilson, C. L.; Sirohey, S. Human and Machine Recognition of Faces: A Survey. Proc. IEEE 1995, 83 (5), 705-740.

(5) Zeng, Z.; Pantic, M.; Roisman, G. I.; Huang, T. S. A Survey of Affect Recognition Methods: Audio, Visual, and Spontaneous Expressions. IEEE Trans. Pattern Anal. Mach. Intell. 2009, 31 (1), 39-58.

(6) Meireles, M. R. G.; Almeida, P. E. M.; Simões, M. G. A Comprehensive Review for Industrial Applicability of Artificial Neural Networks. IEEE Trans. Ind. Electron. 2003, 50 (3), 585-601.

(7) Maltarollo, V. G.; Honório, K. M.; Borges Ferreira da Silva, A. Applications of Artificial Neural Networks in Chemical Problems. In Artificial Neural Networks Architectures and Applications; 2013; pp 203-223.

(8) Brunette, E. S.; Flemmer, R. C.; Flemmer, C. L. A Review of Artificial Intelligence. In ICARA 2009 - Proceedings of the 4th International Conference on Autonomous Robots and Agents; Wellington, New Zealand, 2009; pp 385-392.

(9) Egmont-Petersen, M.; de Ridder, D.; Handels, H. Image Processing with Neural Networks — a Review. Pattern Recognit. 2002, 35 (10), 2279-2301.

(10) Cortes, C.; Vapnik, V. Support-Vector Networks. Chem. Biol. Drug Des. 1995, 20 (3), 273-297. 
(11) Young, M. P.; Yamane, S. Sparse Population Coding of Faces in the Inferotemporal Cortex. Science. 1992, 256 (5061), 1327-1331.

(12) Ngiam, J.; Koh, P.; Chen, Z.; Bhaskar, S.; Ng, A. Sparse Filtering. NIPS 2011, 19.

(13) Baughman, D. R.; Lui, Y. A. Neural Networks in Bioprocessing and Chemical Engineering, 1st ed.; Academic Press: San Diego, 1995.

(14) Gutierrez-osuna, R. Pattern Analysis for Machine Olfaction : A Review. IEEE Sens. J. 2002, 2 (3), 189-202.

(15) Carlo, S. Di; Falasconi, M.; Sánchez, E.; Scionti, A.; Squillero, G.; Tonda, A. Exploiting Evolution for an Adaptive Drift-Robust Classifier in Chemical Sensing; 2010.

(16) Sharma, R. K.; Chan, P. C. H.; Tang, Z.; Yan, G.; Hsing, I. M.; Sin, J. K. O. Investigation of Stability and Reliability of Tin Oxide Thin-Film for Integrated Micro-Machined Gas Sensor Devices. Sensors Actuators, B Chem. 2001, 81 (1), 9-16.

(17) Di Natale, C.; Martinelli, E.; D’Amico, A. Counteraction of Environmental Disturbances of Electronic Nose Data by Independent Component Analysis. Sensors Actuators, B Chem. 2002, 82 (2-3), 158-165.

(18) Roussel, S.; Forsberg, G.; Grenier, P.; Bellon-Maurel, V. Optimisation of Electronic Nose Measurements. Part II: Influence of Experimental Parameters. $J$. Food Eng. 1999, 39 (1), 9-15.

(19) Ionescu, R.; Vancu, a.; Tomescu, a. Time-Dependent Humidity Calibration for Drift Corrections in Electronic Noses Equipped with SnO2 Gas Sensors. Sensors Actuators, B Chem. 2000, 69 (3), 283-286.

(20) Mallya, A. N.; Kottokkaran, R.; Ramamurthy, P. C. Conducting Polymer-Carbon Black Nanocomposite Sensor for Volatile Organic Compounds and Correlating Sensor Response by Molecular Dynamics. Sensors Actuators, B Chem. 2014, 201 (1), 308-320.

(21) Ziyatdinov, A.; Marco, S.; Chaudry, A.; Persaud, K.; Caminal, P.; Perera, A. 
Chemical Drift Compensation of Gas Sensor Array Data by Common Principal Component Analysis. Sensors Actuators B. Chem. 2010, 146 (2), 460-465.

(22) Padilla, M.; Perera, a.; Montoliu, I.; Chaudry, a.; Persaud, K.; Marco, S. Drift Compensation of Gas Sensor Array Data by Orthogonal Signal Correction. Chemom. Intell. Lab. Syst. 2010, 100 (1), 28-35.

(23) Carlo, S. Di; Falasconi, M. Drift Correction Methods for Gas Chemical Sensors in Artificial Olfaction Systems : Techniques and Challenges, Advances in Chemical Sensors. In Advances in Chemical Sensors; Wang, W., Ed.; 2012; pp 305-326.

(24) Severin, E. J.; Doleman, B. J.; Lew, N. S. An Investigation of the Concentration Dependence and Response to Analyte Mixtures of Carbon Black/Insulating Organic Polymer Composite Vapor Detectors. Anal. Chem. 2000, 72 (4), 658-668.

(25) Nakata, S.; Akakabe, S.; Nakasuji, M.; Yoshikawa, K. Gas Sensing Based on a Nonlinear Response: Discrimination between Hydrocarbons and Quantification of Individual Components in a Gas Mixture. Anal. Chem. 1996, 68 (13), 2067-2072.

(26) Kumar, V.; Mishra, V. N.; Dwivedi, R.; Das, R. R. Classification and Quantification of Binary Mixtures of Gases / Odors Using Thick-Film Gas Sensor Array Responses. IEEE Sens. J. 2015, 15 (2), 1252-1260.

(27) Woodka, M. D.; Brunschwig, B. S.; Lewis, N. S. Use of Spatiotemporal Response Information from Sorption-Based Sensor Arrays to Identify and Quantify the Composition of Analyte Mixtures. Langmuir 2007, 23 (26), 13232-13241.

(28) Monroy, J. G.; González-Jiménez, J.; Blanco, J. L. Overcoming the Slow Recovery of MOX Gas Sensors through a System Modeling Approach. Sensors 2012, 12 (10), 13664-13680.

(29) Muezzinoglu, M. K.; Vergara, A.; Huerta, R.; Rulkov, N.; Rabinovich, M. I.; Selverston, A.; Abarbanel, H. D. I. Acceleration of Chemo-Sensory Information Processing Using Transient Features. Sensors Actuators, B Chem. 2009, 137 (2), $507-512$.

(30) Laor, Y.; Parker, D.; Pagé, T. Measurement, Prediction, and Monitoring of Odors in the Environment: A Critical Review. Rev. Chem. Eng. 2014, 30 (2), 139-166. 
(31) Gardner, J. W.; Shin, H. W.; Hines, E. L. An Electronic Nose System to Diagnose Illness. Sensors Actuators B. Chem. 2000, 70 (1), 19-24.

(32) Wilson, A. D.; Baietto, M. Advances in Electronic-Nose Technologies Developed for Biomedical Applications. Sensors 2011, 11 (1), 1105-1176.

(33) Kim, H.; Konnanath, B.; Sattigeri, P.; Wang, J.; Mulchandani, A.; Myung, N.; Deshusses, M. a.; Spanias, A.; Bakkaloglu, B. Electronic-Nose for Detecting Rnvironmental Pollutants: Signal Processing and Analog Front-End Design. Analog Integr. Circuits Signal Process. 2011, 70 (1), 15-32.

(34) Dentoni, L.; Capelli, L.; Sironi, S.; Del Rosso, R.; Zanetti, S.; Della Torre, M. Development of an Electronic Nose for Environmental Odour Monitoring. Sensors 2012, 12 (11), 14363-14381.

(35) Sankaran, S.; Panigrahi, S.; Mallik, S. Odorant Binding Protein Based Biomimetic Sensors for Detection of Alcohols Associated with Salmonella Contamination in Packaged Beef. Biosens. Bioelectron. 2011, 26 (7), 3103-3109.

(36) Sankaran, S.; Panigrahi, S.; Mallik, S. Olfactory Receptor Based Piezoelectric Biosensors for Detection of Alcohols Related to Food Safety Applications. Sensors Actuators, B Chem. 2011, 155 (1), 8-18.

(37) Peris, M.; Escuder-Gilabert, L. A 21st Century Technique for Food Control: Electronic Noses. Anal. Chim. Acta 2009, 638 (1), 1-15.

(38) Panigrahi, S.; Sankaran, S.; Mallik, S.; Gaddam, B.; Hanson, A. a. Olfactory Receptor-Based Polypeptide Sensor for Acetic Acid VOC Detection. Mater. Sci. Eng. C 2012, 32 (6), 1307-1313.

(39) Panigrahi, S.; Balasubramanian, S.; Gu, H.; Logue, C.; Marchello, M. NeuralNetwork-Integrated Electronic Nose System for Identification of Spoiled Beef. LWT - Food Sci. Technol. 2006, 39 (2), 135-145.

(40) Ampuero, S.; Bosset, J. O. The Electronic Nose Applied to Dairy Products: A Review. Sensors Actuators, B Chem. 2003, 94 (1), 1-12.

(41) Sela, L.; Sobel, N. Human Olfaction: A Constant State of Change-Blindness. Exp. Brain Res. 2010, 205 (1), 13-29. 
(42) Buck, L. B. Unraveling the Sense of Smell (Nobel Lecture). Angew. Chem. Int. Ed. 2005, 44 (38), 6128-6140.

(43) Saive, A.-L.; Royet, J.-P.; Ravel, N.; Thévenet, M.; Garcia, S.; Plailly, J. A Unique Memory Process Modulated by Emotion Underpins Successful Odor Recognition and Episodic Retrieval in Humans. Front. Behav. Neurosci. 2014, 8, 203.

(44) Lundström, J. N.; Boesveldt, S.; Albrecht, J. Central Processing of the Chemical Senses: An Overview. ACS Chem. Neurosci. 2011, 2 (1), 5-16.

(45) Ache, B. W.; Young, J. M. Olfaction: Diverse Species, Conserved Principles. Neuron 2005, 48 (3), 417-430.

(46) Lewis, N. S. Comparisons between Mammalian and Artificial Olfaction Based on Arrays of Carbon Black-Polymer Composite Vapor Detectors. Acc. Chem. Res. 2004, 37 (9), 663-672.

(47) Gao, T.; Woodka, M. D.; Brunschwig, B. S.; Lewis, N. S. Chemiresistors for Array-Based Vapor Sensing Using Composites of Carbon Black with Low Volatility Organic Molecules. Chem. Mater. 2006, 22 (18), 5193-5202.

(48) Gutiérrez, J.; Horrillo, M. C. Advances in Artificial Olfaction: Sensors and Applications. Talanta 2014, 124, 95-105.

(49) Benrekia, F.; Attari, M.; Bouhedda, M. Gas Sensors Characterization and Multilayer Perceptron (MLP) Hardware Implementation for Gas Identification Using a Field Programmable Gate Array (FPGA). Sensors 2013, 13 (3), $2967-$ 2985.

(50) Vergara, A.; Fonollosa, J.; Mahiques, J.; Trincavelli, M.; Rulkov, N.; Huerta, R. On the Performance of Gas Sensor Arrays in Open Sampling Systems Using Inhibitory Support Vector Machines. Sensors Actuators B. Chem. 2013, 185, 462477.

(51) Hossain, M. E.; Rahman, G. M. A.; Freund, M. S.; Jayas, D. S.; White, N. D. G.; Shafai, C.; Thomson, D. J. Fabrication and Optimization of a Conducting Polymer Sensor Array Using Stored Grain Model Volatiles. J. Agric. Food Chem. 2012, 60 (11), 2863-2873. 
(52) Gonzalez-Jimenez, J.; Monroy, J. G.; Blanco, J. L. The Multi-Chamber Electronic Nose-An Improved Olfaction Sensor for Mobile Robotics. Sensors 2011, 11 (6), 6145-6164.

(53) Albert, K. J.; Lewis, N. S.; Schauer, C. L.; Sotzing, G. a; Stitzel, S. E.; Vaid, T. P.; Walt, D. R. Cross-Reactive Chemical Sensor Arrays. Chem. Rev. 2000, 100 (7), 2595-2626.

(54) Arshak, K.; Moore, E.; Lyons, G. M.; Harris, J.; Clifford, S. A Review of Gas Sensors Employed in Electronic Nose Applications. Sens. Rev. 2004, 24 (2), 181198.

(55) Doleman, B. J.; Lonergan, M. C.; Severin, E. J.; Vaid, T. P.; Lewis, N. S. Quantitative Study of the Resolving Power of Arrays of Carbon Black-Polymer Composites in Various Vapor-Sensing Tasks. Anal. Chem. 1998, 70 (19), 41774190 .

(56) Freund, M. S.; Lewis, N. S. A Chemically Diverse Conducting Polymer-Based "Electronic Nose”. Proc. Natl. Acad. Sci. U. S. A. 1995, 92 (7), 2652-2656.

(57) Ramesh Kumar, M.; Ryman, S.; Tareq, O.; Buchanan, D. a.; Freund, M. S. Chemical Diversity in Electrochemically Deposited Conducting Polymer-Based Sensor Arrays. Sensors Actuators B Chem. 2014, 202, 600-608.

(58) Iyogun, A. a.; Kumar, M. R.; Freund, M. S. Chemically Diverse Sensor Arrays Based on Electrochemically Copolymerized Pyrrole and Styrene Derivatives. Sensors Actuators B Chem. 2015, 215, 510-517.

(59) Marco, S.; Gutiérrez-gálvez, A. Signal and Data Processing for Machine Olfaction and Chemical Sensing : A Review. IEEE Sens. J. 2012, 12 (11), 3189-3214.

(60) Severin, E. J. Array-Based Vapor Sensing Using Conductive Carbon BlackPolymer Composite Thin Film Detectors, California Institute of Technology: Pasadena, 1999.

(61) Doleman, B. J.; Severin, E. J.; Lewis, N. S. Trends in Odor Intensity for Human and Electronic Noses: Relative Roles of Odorant Vapor Pressure vs. Molecularly Specific Odorant Binding. Proc. Natl. Acad. Sci. U. S. A. 1998, 95 (10), 5442- 
5447.

(62) Briglin, S. M.; Lewis, N. S. Characterization of the Temporal Response Profile of Carbon Black - Polymer Composite Detectors to Volatile Organic Vapors. J. Phys. Chem. B 2003, 107 (40), 11031-11042.

(63) Lonergan, M. C.; Severin, E. J.; Doleman, B. J.; Beaber, S. A.; Grubbs, R. H.; Lewis, N. S. Array-Based Vapor Sensing Using Chemically Sensitive, Carbon Black - Polymer Resistors. Chem. Mater. 1996, 8 (9), 2298-2312.

(64) Vidybida, A. K.; Usenko, A. S.; Kukla, A. L.; Pavluchenko, A. S.; Pokhodenko, V. D. Structural Characteristics of Gas Sensors Based on Conducting Polymer. $J$. Optoelectron. Adv. Mater. 2005, 7 (6), 2815-2821.

(65) McCulloch, W. S.; Pitts, W. A Logical Calculus of the Ideas Immanent in Nervous Activity. Bull. Math. Biophys. 1943, 5 (4), 115-133.

(66) Mao, J. Artificial Neural Networks: A Tutorial. Communications 1996, 29 (3), 3144.

(67) Sammut, C.; Webb, G. Chapter 18 - S. Encyclopedia of Machine Learning; Springer, 2010; $\mathrm{p} 941$.

(68) Sammut, C.; Webb, G. Chapter 20 - U. Encyclopedia of Machine Learning; Springer US, 2010; pp 1001-1009.

(69) Vito, S. De; Fattoruso, G.; Pardo, M.; Tortorella, F.; Member, S.; Francia, G. Di. Semi-Supervised Learning Techniques in Artificial Olfaction : A Novel Approach to Classification Problems and Drift Counteraction. IEEE Sens. J. 2012, 12 (11), $3215-3224$.

(70) Willmore, B.; Tolhurst, D. J. Characterizing the Sparseness of Neural Codes. Netw. Comput. Neural Syst. 2001, 12 (3), 255-270.

(71) Zupan, J. and Gasteiger, J. Neural Networks for Chemists: An Introduction; VCH Publishers: New York, 1993.

(72) Park, J. W.; Jang, J. Fabrication of Graphene/free-Standing Nanofibrillar PEDOT/P(VDF-HFP) Hybrid Device for Wearable and Sensitive Electronic Skin 
Application. Carbon N. Y. 2015, 87, 275-281.

(73) Matzeu, G.; Florea, L.; Diamond, D. Advances in Wearable Chemical Sensor Design for Monitoring Biological Fluids. Sensors Actuators B Chem. 2015, 211, 403-418.

(74) Bosiers, J. T.; Peters, I. M.; Draijer, C.; Theuwissen, A. Technical Challenges and Recent Progress in CCD Imagers. Nucl. Instruments Methods Phys. Res. Sect. A Accel. Spectrometers, Detect. Assoc. Equip. 2006, 565 (1), 148-156.

(75) Abraham, H. Solubility Interactions and the Design of Chemically Selective Sorbent Coatings for Chemical Sensors and Arrays. Sensors Actuators B Chem. 1991, 3 (2), 85-111.

(76) Magda, B.; de Gennaro, G.; de Pinto, V.; Demarinis Loiotile, A.; Lovascio, S.; Penza, M. Odour Detection Methods: Olfactometry and Chemical Sensors. Sensors 2011, 11 (5), 5290-5322.

(77) Di Carlo, S.; Falasconi, M.; Sanchez, E.; Sberveglieri, G.; Scionti, A.; Squillero, G.; Tonda, A.; Gouma, P. Covariance Matrix Adaptation Evolutionary Strategy for Drift Correction of Electronic Nose Data. In Olfaction and Electronic Nose: Proceedings of the 14th International Symposium on Olfaction and Electronic Nose; 2011; Vol. 1362, pp 25-26.

(78) Garg, T.; Porwal, R. A Survey on Sensor's Drift Counteraction Using Dynamic Pattern Recognition System . Int. J. Comput. Sci. Eng. 2011, 3 (1), 127-132.

(79) Zhang, L.; Tian, F.; Liu, S.; Dang, L.; Peng, X.; Yin, X. Chaotic Time Series Prediction of E-Nose Sensor Drift in Embedded Phase Space. Sensors Actuators $B$ Chem. 2013, 182, 71-79.

(80) Carlo, S. Di; Falasconi, M.; Sanchez, E.; Scionti, A.; Squillero, G.; Tonda, A. Increasing Pattern Recognition Accuracy for Chemical Sensing by Evolutionary Based Drift Compensation. Pattern Recognit. Lett. 2011, 32 (13), 1594-1603.

(81) Liu, T.; Chaibou, K.; Huang, Z. A Novel Retraining Method of Multiple SelfOrganizing Maps for Gas Sensor Drift Compensation. Sensors Mater. 2013, 25 (2), 109-120. 
(82) Vergara, A.; Vembu, S.; Ayhan, T.; Ryan, M. a.; Homer, M. L.; Huerta, R. Chemical Gas Sensor Drift Compensation Using Classifier Ensembles. Sensors Actuators B Chem. 2012, 166-167, 320-329.

(83) Polese, D.; Martinelli, E.; D’Amico, a.; Di Natale, C. An Olfactory Bulb Model Mitigates the Drift in Chemical Sensors. Procedia Eng. 2012, 47, 1069-1072.

(84) Lundberg, B.; Sundqvist, B. Resistivity of a Composite Conducting Polymer as a Function of Temperature, Pressure, and Environment: Applications as a Pressure and Gas Concentration Transducer. J. Appl. Phys. 1986, 60 (3), 1074-1079.

(85) Marques, L.; Nunes, U.; T. de Dealmeida, A. Olfaction-Based Mobile Robot Navigation. Thin Solid Films 2002, 418 (1), 51-58.

(86) Niessing, J.; Friedrich, R. W. Olfactory Pattern Classification by Discrete Neuronal Network States. Nature 2010, 465 (7294), 47-52.

(87) Devos, M. Patte, F. Rouault, J. Laffort, P. Van Gemert, L. J. Standardized Human Olfactory Thresholds; Oxford University Press: New York, 1990.

(88) Doty, R. L.; Shaman, P.; Dann, M. Development of the University of Pennsylvania Smell Identification Test: A Standardized Microencapsulated Test of Olfactory Function. Physiol. Behav. 1984, 32 (3), 489-502.

(89) Purves, D.; Fitzpatrick, D.; Katz, L. C.; Lamantia, A.-S.; McNamara, J. O.; Williams, S. M.; Augustine, G. J. Olfactory Perception in Humans, 2nd ed.; Sinauer Associates, 2001.

(90) Smyth, G. K. Pearson's Goodness of Fit Statistic as a Score Test Statistic. In Lecture Notes--Monograph Series; 2003; Vol. 40, pp 115-126.

(91) Version 15.10, M. S. bvba. MedCalc https://www.medcalc.org/manual/chi-squaretable.php (accessed Jan 1, 2015).

(92) Kamlet, M. J.; Abboud, J.-L. M.; Abraham, M. H.; Taft, R. W. Linear Solvation Energy Relationships. 23. A Comprehensive Collection of the Solvatochromic Parameters, $\pi^{*}, \alpha$, and $\beta$, and Some Methods for Simplifying the Generalized Solvatochromic Equation. J. Org. Chem 1983, 48 (17), 2877-2887. 
(93) Lewis, N. S. Comparisons between Mammalian and Artificial Olfaction Based on Arrays of Carbon Black-Polymer Composite Vapor Detectors. Acc. Chem. Res. 2004, 37 (9), 663-672.

(94) Sobel, N.; Khan, R. M.; Saltman, a; Sullivan, E. V; Gabrieli, J. D. The World Smells Different to Each Nostril. Nature 1999, 402 (6757), 35.

(95) Frasnelli, J.; Charbonneau, G.; Collignon, O.; Lepore, F. Odor Localization and Sniffing. Chem. Senses 2009, 34 (2), 139-144.

(96) Porter, J.; Anand, T.; Johnson, B.; Khan, R. M.; Sobel, N. Brain Mechanisms for Extracting Spatial Information from Smell. Neuron 2005, 47 (4), 581-592.

(97) Hossain, M. E. Fabrication and Optimization of a Sensor Array for Incipient Grain Spoilage Monitoring, University of Manitoba, 2010.

(98) Keller, A. Attention and Olfactory Consciousness. Front. Psychol. 2011, 2 (380), $1-13$.

(99) Hopfield, J. Olfactory Computation and Onject Perception. Neurobiology 1991, 88 (15), 6462-6466.

(100) Pardo, M.; Faglia, G.; Sberveglieri, G.; Corte, M.; Masulli, F.; Riani, M. Monitoring Reliability of Sensors in an Array by Neural Networks. Sensors Actuators B. Chem. 2000, 67, 128-133.

(101) Ziyatdinov, A.; Perera, A. Synthetic Benchmarks for Machine Olfaction: Classification, Segmentation and Sensor Damage. Data Br. 2015, 3, 126-130.

(102) Briglin, S. M.; Freund, M. S.; Tokumaru, P.; Lewis, N. S. Exploitation of Spatiotemporal Information and Geometric Optimization of Signal/noise Performance Using Arrays of Carbon Black-Polymer Composite Vapor Detectors. Sensors Actuators B Chem. 2002, 82 (1), 54-74. 